\title{
Anomalies and supersymmetry
}

\author{
Ruben Minasian $\odot,{ }^{1, *}$ Ioannis Papadimitriou $\odot,{ }^{2, \dagger}$ and Piljin Yi ${ }^{2, 末}$ \\ ${ }^{1}$ Institut de Physique Théorique, Université Paris Saclay, CNRS, CEA, F-91191 Gif-sur-Yvette, France \\ ${ }^{2}$ School of Physics, Korea Institute for Advanced Study, Seoul 02455, Korea
}

(Received 12 January 2022; accepted 10 February 2022; published 7 March 2022)

\begin{abstract}
We revisit quantum field theory anomalies, emphasizing the interplay with diffeomorphisms and supersymmetry. The Ward identities of the latter induce Noether currents of all continuous symmetries, and we point out how these consistent currents are replaced by their covariant form through the appearance of the Bardeen-Zumino currents, which play a central role in our study. For supersymmetry Ward identities, two systematic methods for solving the Wess-Zumino consistency conditions are discussed: anomaly inflow and anomaly descent. The simplest inflows are from supersymmetric ChernSimons actions in one dimension higher, which are used to supersymmetrize flavor anomalies in $d=4$ and, for $d=2 \mathcal{N}=(p, q)$, flavor anomalies with $p, q \leq 3$ and Lorentz-Weyl anomalies with $p, q \leq 6$. Finally, we extend the Becchi-Rouet-Stora-Tyutin algebra and the subsequent descent, a necessity for the diffeomorphism anomaly in retrospect. The same modification computes the supersymmetrized anomalies and determines the above Chern-Simons actions when these exist.
\end{abstract}

DOI: 10.1103/PhysRevD.105.065005

\section{INTRODUCTION AND SUMMARY OF RESULTS}

Perturbative anomalies [1-3] are well understood as a failure of the path integral measure to respect the symmetry in question [4]. Perhaps the most succinct way to compute and phrase the anomaly is by gauging the would-be symmetry and consider the symmetry transformation of the effective action after integrating out the chiral fields responsible for the anomaly. A most comprehensive computation of this kind was given in Ref. [5]. Further subtleties, such as the distinction between consistent and covariant currents, diffeomorphisms, and Lorentz transformations, were addressed by Ref. [6].

Through these developments, we are accustomed to treating gravitational or diffeomorphism anomalies on an equal footing with those associated with internal symmetries, such as gauge or flavor symmetries. More often than not, instead of the actual diffeomorphism anomaly we compute what is better referred to as a Lorentz anomaly under an $S O(d)$ gauge rotation of the spin connection $\omega$,

$$
\delta \omega^{a}{ }_{b}=\mathrm{d} L^{a}{ }_{b}+\omega^{a}{ }_{c} L^{c}{ }_{b}-L^{a}{ }_{c} \omega^{c}{ }_{b}
$$

\footnotetext{
*ruben.minasian@ipht.fr

ioannis@kias.re.kr

piljin@kias.re.kr
}

Published by the American Physical Society under the terms of the Creative Commons Attribution 4.0 International license. Further distribution of this work must maintain attribution to the author(s) and the published article's title, journal citation, and DOI. Funded by SCOAP.
Although the equivalence of the gravitational and Lorentz anomalies has been established a long time ago [6], their precise relation is far from obvious, given that diffeomorphisms involve both $S O(d)$ rotations and translations.

Diffeomorphisms act via the Lie derivative along a vector field, say $\xi$. They induce a Lorentz transformation, parametrized by $L$ in (1.1), via $\nabla \xi$, but also involve a translational shift of $\omega$. As we review in Sec. III, despite this difference, the diffeomorphism anomaly is still computable by a $G L(d)$ anomaly descent on the Christoffel symbol $\Gamma$, suitably elevated to a 1-form connection. This helps establish the equivalence between these two anomalies, which justifies the usual focus on the Lorentz anomaly, especially when one is only interested in the question of anomaly cancellation.

When it comes to the anomalous Ward identities with nonvanishing anomalies, however, we must be more attentive to such differences. After all, Lorentz rotations and diffeomorphisms are two different operations. The diffeomorphism Ward identity involves not only the divergence of the energy-momentum tensor, but also all other symmetry currents, since the Lie derivative acts on currents universally, which can be in turn converted to a transformation of the associated (external) gauge fields via the path integral. Of course, the converse is not true, since gauge/flavor rotations do not affect the energy-momentum tensor.

Two distinct anomalous currents are often discussed: consistent and covariant [6]. The former arise from a direct variation of the effective action. The difference between the currents is given by the Bardeen-Zumino (BZ) current, a local quantity built out of the external gauge fields and 
determined entirely by the anomaly descent. In the literature, its use is mostly restricted to helping clarify the relation between different computations of anomalies. However, as we will see, it plays a central role in our discussion.

Since the consistent current is the one that couples to the gauge field, it is often considered as more physical, while its covariant counterpart has played a relatively minor role. On the other hand, it is not difficult to see that the diffeomorphism Ward identity is invariant under such gauge/flavor symmetries, and so are the energy-momentum tensor and the diffeomorphism anomaly. So, even though the consistent currents of such internal symmetries appear naturally in the path integral derivation of the diffeomorphism Ward identity, they must somehow be replaced by their covariant counterparts to ensure the gauge/flavor invariance of the diffeomorphism Ward identity. The way the BZ terms that relate the consistent and covariant gauge/flavor currents arise from a rearrangement of the diffeomorphism Ward identity is the main subject of Sec. III.

Another important Ward identity which generally involves other symmetry currents is that of supersymmetry. Since the square of supersymmetry generates translations or diffeomorphisms, it is not surprising that the two suffer from some common issues. ${ }^{1}$ Supersymmetry acts on all gauge fields, external or internal, and as a consequence the conservation of the supercurrent, as in the diffeomorphism Ward identity, involves all gauge/flavor currents present. In a supersymmetric theory with anomalous internal symmetries, one naturally elevates the accompanying gauge fields to vector supermultiplets. As a result, the supersymmetry Ward identity involves all operators in the corresponding current supermultiplets. In Sec. IV we demonstrate that, as with diffeomorphisms, the BZ current terms are generated and all consistent currents in the supersymmetry Ward identity are eventually replaced by their covariant counterparts.

However, there are two related important differences between diffeomorphisms and supersymmetry in this respect. First, the presence of gauge/flavor anomalies does not violate the diffeomorphism or Lorentz invariance of the effective action, nor does it generate unexpected contributions to the diffeomorphism Ward identity. In this case, the covariantization of the current occurs via rearrangement of existing terms. As we discuss in Sec. IV, this is not the case for supersymmetry in the Wess-Zumino (WZ) gauge for the gauge multiplet. In this gauge, which is sometimes unavoidable, any perturbative anomaly necessarily leads to noninvariance of the effective action under rigid supersymmetry, an observation

\footnotetext{
${ }^{1} R$ symmetry share features with both gauge/flavor symmetries and supersymmetry/diffeomorphisms. For rudimentary discussions, it may be treated as one of the former but not so when we get down to details of how it enters the Ward identities in question. We will try to indicate such differences explicitly as it becomes necessary in later parts of this paper.
}

dating back to the 1980s [7-19] and often referred to as a "supersymmetry anomaly." An analogous observation in the presence of a gravitational anomaly in two dimensions was first made in Refs. [20,21]. Locally supersymmetric contributions to gauge/flavor and $R$-symmetry anomalies were pointed out in Refs. [22,23] and computed explicitly more recently in Refs. [24-32]. See also Refs. [33-35] for a classification of supersymmetrized supergravity anomalies.

The second difference between supersymmetry and diffeomorphisms is that the former does not have an independent solution to the WZ consistency conditions. One might naively think that the supersymmetry Ward identity must be thus simpler than that of diffeomorphisms, yet the situation is actually the opposite. In the supersymmetry Ward identities, the leading "anomalous" term due to any other types of anomalies, gauge/flavor, $\mathrm{R}$ symmetry, and diffeomorphisms, is given by the BZ current contributions that covariantize the consistent currents thereof. Such a noncovariant term could have been expected on general grounds, given that the supersymmetry Ward identity should be invariant under these other symmetries. However, this shift by the BZ current alone does not solve all WZ consistency conditions, and so on the right-hand side of the Ward identity one encounters additional invariant pieces, involving the superpartners of the gauge fields.

These two differences are also behind the fact that the mechanism for the appearance of the BZ currents for gauge/flavor (and $R$ symmetries) in the supersymmetry and diffeomorphism Ward identities is not exactly the same. As we explain in detail in Secs. III and IV, the covariantization of the currents in the latter arises because the diffeomorphism Ward identity contains a linear combination of the anomalous Ward identities for all gauge/flavor symmetries (and $R$ symmetries). In both cases, nevertheless, the covariantization of the currents is facilitated by the fundamental relation between the BZ current and the corresponding anomaly.

A superspace description, when it exists, ensures that the supersymmetry invariance of the effective action may be restored by extending the multiplet of currents to a larger one $[19,29,31,32]$. This, however, does not change the actual content of the Ward identity; it merely gives different names to the same local terms. Furthermore the WZ gauge for the gauge fields is often a necessity, as for theories with extended supersymmetries. The WZ gauge is often appropriate and sometimes an unavoidable framework for computing (refined) physical observables in supersymmetric theories using, e.g., supersymmetric localization. It was in this context that the supersymmetrized form of the gauge/flavor and, in particular, $R$-symmetry anomalies were recently rediscovered [24] and their consequences for supersymmetric partition functions explored [36]. We will refer to these phenomena as "supersymmetrized 
anomaly" or "supersymmetric completion of anomaly" in most of this paper. $^{2}$

Supersymmetrization of various anomalies is a direct consequence of the WZ consistency conditions in supersymmetric theories and ought to fit naturally within established methods for solving these, such as the descent formalism [6,37-39] and, more physically, the anomaly inflow [40].

Anomaly inflow provides a cancellation mechanism for gauge/flavor, $R$ symmetry, and gravitational anomalies via, for example, the symmetry transformation of a ChernSimons action in one dimension higher. It is only natural that a supersymmetric Chern-Simons action, if it exists, cancels all components of the supersymmetrized anomalies. In Sec. V we show that this is indeed the case and, turning the argument around, we use anomaly inflow as a powerful tool to compute supersymmetric anomalies in theories with extended rigid or local supersymmetry.

Anomaly descent provides a more abstract approach to the solution of the WZ consistency conditions. An interesting question in this context is how the interplay between internal and spacetime symmetries is reflected in the descent procedure. The standard anomaly descent applies to gauge/flavor symmetries, as well as local Lorentz and $R$ symmetry, but it becomes less obvious already for diffeomorphisms, given that these generate translations as well as $G L(d)$ rotations. Supersymmetry adds a further layer of complication. Remarkably, as we show in Sec. VI, diffeomorphisms and supersymmetry can be accommodated by a single generalization of the standard descent procedure.

In both anomaly inflow and descent, Chern-Simons actions in one dimension higher have a prominent role. In anomaly descent, they arise from the anomaly polynomial as an intermediate step in the computation of the consistent anomaly, while they provide the simplest anomaly inflow mechanism, canceling the anomaly on a codimension-one boundary or defect. In both cases, the BZ current emerges from the Chern-Simons action via the so-called antiderivative operation, providing the key ingredient for converting the consistent currents in the diffeomorphism and supersymmetry Ward identities to covariant ones.

The rest of the paper is organized as follows. We start with a preliminary review of the effective action, anomalies, and the standard anomaly descent in Sec. II, highlighting the role of the BZ current. We also revisit the standard anomaly descent procedure and the corresponding Becchi-RouetStora-Tyutin (BRST) algebra as a warm-up to our subsequent analysis. In Sec. III we review the diffeomorphism anomaly and contrast it with the Lorentz one. In particular, we explain why the anomaly descent of the Christoffel connection computes the former, while that of the spin connection computes the Lorentz anomaly. However, a

\footnotetext{
${ }^{2}$ The latter should not be mistaken to imply that the anomalies under consideration are part of an anomaly multiplet.
}

discussion of the BRST mechanism that underlies this distinction is deferred to Sec. VI, where we present the anomaly descent and the BRST algebra in a more general context that allows us to accommodate supersymmetry as well.

Section IV addresses some general features of the supersymmetry Ward identity and, in particular, how the anomalies associated with other symmetries give rise to new terms. The BZ current appears precisely due to these terms, and covariantizes the relevant consistent currents of gauge/flavor symmetries. Unlike the diffeomorphism case, however, it does not stop there. The WZ consistency conditions demand further contributions with more gauginos that are gauge/ flavor invariant. An interesting question is what should happen exactly to these induced anomalous terms if some external inflow mechanism is introduced to cancel the gauge/ flavor anomaly to begin with. Generally, the natural expectation that inflow must cancel all such terms is confirmed up to the BZ term, showing that the supersymmetry transformation of the effective action becomes gauge invariant when combined with the bulk inflow term.

Section V takes up the remaining question of Sec. IV, on the gauge/flavor invariant part of the induced anomalous terms. We show that, in the case of a codimension-one inflow from a supersymmetric Chern-Simons action, the cancellation is complete and the combined action, i.e., the effective action and the bulk supersymmetric Chern-Simons action is invariant under supersymmetry transformations as long as the underlying gauge/flavor anomaly is canceled. This also means that one can compute the entire supersymmetrized anomaly simply from the variation of a ChernSimons action in one dimension higher, with appropriate supersymmetry, which naturally becomes a boundary term. Such a codimension-one inflow is not the most general form of inflow, but when it exists, it presents perhaps the most efficient way to compute the supersymmetric completion.

Finally, we come back to the anomaly descent, or more precisely the BRST algebra thereof, in Sec. VI, and extend the standard BRST algebra to accommodate these phenomena systemically. Although not widely recognized, this modification is actually necessary to elevate the WZ consistency conditions for diffeomorphisms to the BRST algebra. A key observation is that the content of the BRST gauge field does not need to match precisely the structure of the BRST operator in order for a descent mechanism to emerge and provide a solution of the WZ consistency conditions. One can get a first glimpse of the necessity of this from the diffeomorphism anomaly descent. The BRST transformation involves both translations and rotations, while the usual descent involves ghosts for the rotational part, say the local Lorentz transformation, only.

This extension proves essential also in the context of supersymmetry, and we use this formulation to determine the general structure of supersymmetrized anomalies. Up to the very first response of the anomaly to supersymmetry, 
which is expressed in terms of the BZ current and described in detail in Sec. IV, the descent procedure is universal and independent of spacetime dimension. The additional gauge/ flavor invariant terms are sensitive to the precise supersymmetry multiplet and spacetime dimension and are left implicit. We end Sec. VI by demonstrating that for certain multiplets the generalized descent determines the supersymmetric Chern-Simons action responsible for the inflow mechanism in Sec. V. We have tried to keep the discussion in Sec. VI general and minimal, but for completeness we have collected some useful related notions that have appeared in the past literature in the Appendix.

\section{AN OVERVIEW OF ANOMALIES}

While gauge anomalies and anomalies of global symmetries come with very different consequences, the classification and computation of anomalies does not really distinguish between the two classes. Since these anomalies arise from the path integral of chiral fields, be they fermions or self-dual tensors, rather than of the gauge fields, we may as well compute these anomalies on an equal footing by considering the relevant gauge fields to be all external and the symmetry to be global. Only at the end of computation, do we worry about the cancellation of the anomaly if the relevant symmetry is gauged. Much of what we review here can be traced back to Ref. [6].

\section{A. Effective action and anomalous Ward identities}

For a general discussion, let us introduce the effective action $W(\mathcal{A})$ which is a result of path integrals of all chiral fields coupled to the "gauge fields," collectively denoted as $\mathcal{A}$,

$$
e^{-W(\mathcal{A})}=\int[\mathcal{D} \Psi] e^{-S(\Psi ; \mathcal{A})},
$$

where the chiral fields responsible for the anomaly are denoted collectively by $\Psi$. Suppose that the action $S(\Psi$; $\mathcal{A}=0$ ) is invariant under a global symmetry $\delta$ acting on $\Psi$.

This can be then formally elevated to a local symmetry of $S(\Psi ; \mathcal{A})$ with the gauge field $\mathcal{A}$ introduced, so that

$$
S(\Psi+\delta \Psi ; \mathcal{A}+\delta \mathcal{A})=S(\Psi ; \mathcal{A}),
$$

or equivalently

$$
\begin{aligned}
& S(\Psi+\delta \Psi ; \mathcal{A})-S(\Psi ; \mathcal{A}) \\
& =S(\Psi ; \mathcal{A}-\delta \mathcal{A})-S(\Psi ; \mathcal{A}) \\
& =-\delta \mathcal{A} \cdot \mathcal{J}
\end{aligned}
$$

at the linear order, with the sign convention

$$
\frac{\delta S}{\delta \mathcal{A}}=\mathcal{J}
$$

If the path integral measure is invariant under such transformations as well, we have

$$
\begin{aligned}
\int[\mathcal{D} \Psi] e^{-S(\Psi ; \mathcal{A})} & =\int[\mathcal{D}(\Psi+\delta \Psi)] e^{-S(\Psi+\delta \Psi ; \mathcal{A}+\delta \mathcal{A})} \\
& =\int[\mathcal{D} \Psi] e^{-S(\Psi ; \mathcal{A}+\delta \mathcal{A})}
\end{aligned}
$$

where, for the second equality, we used that $\Psi$ is a dummy variable for the integral. However, the anomaly arises precisely because the path integral measure is not invariant, so the very first step fails.

A gauge transformation $\delta_{\Phi}$, say, $\delta_{\Phi} \mathcal{A}=\mathrm{d} \Phi+\cdots$, induces

$$
\delta_{\Phi} W(\mathcal{A})=\delta_{\Phi} \mathcal{A} \cdot \frac{\delta W}{\delta \mathcal{A}}=-\Phi \cdot\left\langle\nabla_{\mu} \mathcal{J}^{\mu}\right\rangle,
$$

whose anomalous value is supposed to be captured by the so-called consistent anomaly,

$$
\Phi \cdot\left\langle\nabla_{\mu} \mathcal{J}^{\mu}\right\rangle=\int \mathbf{w}_{d}^{(1)}(\Phi ; \mathcal{A}),
$$

where $\mathbf{w}_{d}^{(1)}$ is a local functional obtained from anomaly descent. Throughout this paper we denote these standard anomalies by $G(\Phi ; \mathcal{A})$. We will give a brief review of the relevant manipulations in the following subsection.

More generally, however, external gauge fields of one symmetry might interfere with such a Ward identity for some other symmetries. The simplest example of this is diffeomorphisms $\delta_{\xi} ; \delta_{\xi} W$ can be expressed generally as

$$
\delta_{\xi} W=\delta_{\xi} \Gamma \cdot \frac{\delta W}{\delta \Gamma}+\sum_{\mathcal{A}^{\prime} \neq \Gamma} \delta_{\xi} \mathcal{A}^{\prime} \cdot \frac{\delta W}{\delta \mathcal{A}^{\prime}},
$$

where the sum is over the other external gauge fields. $\Gamma$ is the Christoffel connection; $\delta_{\xi} \Gamma$ is qualitatively different from ordinary gauge transformations since it involves translational components as well. See Sec. III A.

In terms of the path integral we have

$$
\begin{aligned}
\delta_{\xi} e^{-W}= & \int[\mathcal{D} \Psi]\left(\xi_{\nu} \cdot \nabla_{\mu} T^{\mu \nu}(\Psi)\right. \\
& \left.-\sum^{\prime} \delta_{\xi} \mathcal{A}^{\prime} \cdot \mathcal{J}^{\prime}(\Psi)\right) e^{-S\left(\Psi ; \Gamma, \mathcal{A}^{\prime}\right)}
\end{aligned}
$$

with the energy-momentum tensor $T_{\mu \nu}$. What is the path integral interpretation of this expression? Note that $\xi_{\nu}$. $\nabla_{\mu} T^{\mu \nu}(\Psi)$ downstairs is constructed out of the dynamical fields and acts on all things made up of $\Psi$. Since all currents are vectorial, the operator $\nabla_{\mu} T^{\mu \nu}$ acts on $\mathcal{J}^{\prime}$ of the $\mathcal{A}^{\prime} \cdot \mathcal{J}^{\prime}$ term as well, which means that $\xi_{\nu} \cdot \nabla_{\mu} T^{\mu \nu}(\Psi)$ by itself will not leave the effective action invariant, even in the absence of an anomaly, but a counteracting rotation of $\mathcal{A}$ must 
accompany the transformation. The second term does exactly this, so that $-\delta_{\xi} W$ is the quantity that is captured by the conventional anomaly, leading to the Ward identity

$$
\xi_{\nu} \cdot\left\langle\nabla_{\mu} T^{\mu \nu}\right\rangle-\sum^{\prime} \delta_{\xi} \mathcal{A}^{\prime} \cdot\left\langle\mathcal{J}^{\prime}\right\rangle=G_{\mathrm{diffeo}}(\xi ; \mathcal{A}) .
$$

Later we will also review the diffeomorphism anomaly $G_{\text {diffeo }}(\xi ; \mathcal{A})$. As such, it is important to keep track of the difference between the anomaly $\delta W$ and the divergence of the would-be-conserved current $\left\langle\nabla_{\mu} \mathcal{J}^{\mu}\right\rangle$.

\section{B. Anomaly descent and Bardeen-Zumino currents}

The anomaly descent solves the following functional equation for a local functional $G(\Phi ; \mathcal{A})$ :

$$
\delta_{\Phi_{1}} \delta_{\Phi_{2}} W-\delta_{\Phi_{2}} \delta_{\Phi_{1}} W=\delta_{\left[\Phi_{1}, \Phi_{2}\right]} W
$$

where $\delta_{\Phi}$ is a gauge transformation of the relevant connection $\mathcal{A}$. This comes with the sign choice

$$
\delta_{\Phi} \mathcal{A}=\mathrm{d} \Phi+[\mathcal{A}, \Phi], \quad \delta_{\Phi} \mathcal{F}=[\mathcal{F}, \Phi],
$$

and the convention that $\delta$ does not act on the gauge parameters, so that $\delta_{\Phi_{1}} \delta_{\Phi_{2}} \mathcal{F}=\left[\left[\mathcal{F}, \Phi_{1}\right], \Phi_{2}\right]$. One could have various different conventions such as $\delta$ acts on parameters, which could be more natural depending precisely on the computation at hand.

In the BRST formulation [41], we replace $\Phi$ by a Grassmann odd gauge function $v$ and recast the gauge transformation into the anticommuting version $\mathbf{s}$ which acts as

$$
\begin{aligned}
\mathbf{s} \mathcal{A} & =-\mathrm{d} v-\mathcal{A} v-v \mathcal{A}, \\
\mathbf{s} \mathcal{F} & =\mathcal{F} v-v \mathcal{F}, \\
\mathbf{s} v & =-v^{2} .
\end{aligned}
$$

We treat $\mathrm{d}$ and $\mathbf{s}$ on an equal footing, and the same with $\mathcal{A}$ and $v$, and assign BRST odd grading on all of these. One can recover, say, from $\mathbf{s} \mathcal{A}$, the usual gauge transformation $\delta_{\Phi} \mathcal{A}=\mathrm{d} \Phi+\cdots$ if we write $v=\chi \Phi$ and pull the Grassmann odd constant $\chi$ all the way to the left. The same happens with $\mathbf{s} \mathcal{F}$ since $\mathcal{F}$ is a 2 -form and thus even.

The standard anomaly descent is based on these nilpotent operators $\mathrm{d}, \mathbf{s}$, and $\mathrm{d}+\mathbf{s}$, which obey

$$
\mathrm{d}^{2}=0, \quad \mathbf{s}^{2}=0, \quad(\mathrm{~d}+\mathbf{s})^{2}=0 .
$$

Recall that the nilpotency of $\mathrm{d}$ implies that the field strength $\mathcal{F} \equiv \mathrm{d} \mathcal{A}+\mathcal{A}^{2}$ satisfies the Bianchi identity $\mathrm{d} \mathcal{F}+\mathcal{A} \mathcal{F}-\mathcal{F} \mathcal{A}=0$. The two key observations were the "Russian formula" of [39]

$$
\hat{\mathcal{F}} \equiv(\mathrm{d}+\mathbf{s}) \hat{\mathcal{A}}+\hat{\mathcal{A}}^{2}=\mathcal{F}, \quad \hat{\mathcal{A}} \equiv \mathcal{A}+v,
$$

and the generalized Bianchi identity

$$
(\mathrm{d}+\mathbf{s}) \hat{\mathcal{F}}+\hat{\mathcal{A}} \hat{\mathcal{F}}-\hat{\mathcal{F}} \hat{\mathcal{A}}=0
$$

The final ingredient is an anomaly polynomial $P_{d+2}$ such that

$$
0=\mathrm{d} P_{d+2}(\mathcal{F}),
$$

and the related Chern-Simons form $\mathbf{w}_{d+1}(\mathcal{A}, \mathcal{F})$ that satisfies $P_{d+2}(\mathcal{F})=\mathrm{d} \mathbf{w}_{d+1}(\mathcal{A}, \mathcal{F})$. Similarly, the same polynomial in $\hat{\mathcal{F}}, P_{d+2}(\hat{\mathcal{F}})$ is $(\mathrm{d}+\mathbf{s})$ closed.

One generates a solution to the WZ consistency condition by considering an expansion in $v$ of the right-hand side of the identity

$$
\begin{aligned}
P_{d+2}(\mathcal{F})= & P_{d+2}(\hat{\mathcal{F}}) \\
= & (\mathrm{d}+\mathbf{s}) \mathbf{w}_{d+1}(\mathcal{A}+v, \mathcal{F}) \\
= & P_{\mathrm{d}+2}(\mathcal{F})+\mathbf{s} \mathbf{w}_{d+1}^{(0)}(\mathcal{A}, \mathcal{F})+\mathrm{d}_{d}^{(1)}(v ; \mathcal{A}, \mathcal{F}) \\
& +O\left(v^{2}\right)
\end{aligned}
$$

where the numeral superscript keeps track of the power of $v$,

$$
\mathbf{w}_{d+1}(\mathcal{A}+v, \mathcal{F})=\sum_{k \geq 0} \mathbf{w}_{d+1-k}^{(k)}(v ; \mathcal{A}, \mathcal{F}) .
$$

As noted above, the translation back to the bosonic version requires $v=\chi \Phi$ and moving the Grassmann odd piece $\chi$ all the way to the left in all expressions. For example,

$$
\begin{aligned}
\chi \delta_{\Phi} \mathbf{w}_{d+1}(\mathcal{A}, \mathcal{F}) & =\left.\mathbf{s w}_{d+1}^{(0)}(\mathcal{A}, \mathcal{F})\right|_{v \rightarrow \chi \Phi} \\
& =-\mathrm{d}_{d}^{(1)}(\chi \Phi ; \mathcal{A}, \mathcal{F}) \\
& =\chi \mathrm{d} \mathbf{w}_{d}^{(1)}(\Phi ; \mathcal{A}, \mathcal{F}) .
\end{aligned}
$$

At order $v^{2}$ the equality in (2.18) yields

$$
\mathbf{s} \int \mathbf{w}_{d}^{(1)}(v ; \mathcal{A}, \mathcal{F})=-\int \mathrm{d}\left(\mathbf{w}_{d-1}^{(2)}(v ; \mathcal{A}, \mathcal{F})\right)=0,
$$

which implies that the integral of $\mathbf{w}_{d}^{(1)}$ gives the desired solution to the consistency condition.

To see this it suffices to take $v=\chi_{1} \Phi_{1}+\chi_{2} \Phi_{2}$ with Grassmann odd, $\chi_{1} \neq \chi_{2}$. $\delta_{\Phi}$ acts only on fields, while $\mathbf{s}$ is designed to act on $v$ as well, such that the kernel of $\mathbf{s}$ solves the WZ consistency condition, modulo exact terms. This brings us to the usual anomaly descent solving the consistency condition,

$$
-\delta_{\Phi} W=G(\Phi ; \mathcal{A}) \equiv \int \mathbf{w}_{d}^{(1)}(\Phi ; \mathcal{A}, \mathcal{F})
$$


with

$$
P_{d+2}(\mathcal{F})=\mathrm{d} \mathbf{w}_{d+1}(\mathcal{A}, \mathcal{F})
$$

$$
\delta_{\Phi} \mathbf{w}_{d+1}(\mathcal{A}, \mathcal{F})=\mathrm{d} \mathbf{w}_{d}^{(1)}(\Phi ; \mathcal{A}, \mathcal{F})
$$

via an entirely bosonic descent procedure.

A well-known ambiguity in this procedure occurs in the very first step, when a mixed term of type $P_{2 n}\left(\mathcal{F}_{1}\right) \wedge P_{d+2-2 n}\left(\mathcal{F}_{2}\right)$ is present in the anomaly polynomial. The right-hand side has the ambiguity

$$
\cdots+\alpha \mathbf{w}_{2 n-1}\left(\mathcal{A}_{1}, \mathcal{F}_{1}\right) \wedge P_{d+2-2 n}\left(\mathcal{F}_{2}\right)+\beta P_{2 n}\left(\mathcal{F}_{1}\right) \wedge \mathbf{w}_{d+1-2 n}\left(\mathcal{A}_{2}, \mathcal{F}_{2}\right)+\cdots
$$

with $\alpha+\beta=0$, so that

$$
\begin{aligned}
& \mathbf{w}_{d}^{(1)}\left(\Phi_{1} ; \mathcal{A}, \mathcal{F}\right)=\cdots+\alpha \mathbf{w}_{2 n-2}^{(1)}\left(\Phi_{1}, \mathcal{A}_{1}, \mathcal{F}_{1}\right) \wedge P_{d+2-2 n}\left(\mathcal{F}_{2}\right)+\cdots, \\
& \mathbf{w}_{d}^{(1)}\left(\Phi_{2} ; \mathcal{A}, \mathcal{F}\right)=\cdots+\beta P_{2 n}\left(\mathcal{F}_{1}\right) \wedge \mathbf{w}_{d-2 n}^{(1)}\left(\Phi_{2} ; \mathcal{A}_{2}, \mathcal{F}_{2}\right)+\cdots
\end{aligned}
$$

The ambiguity is a matter of a local counterterm in $d$ dimensions, so one can choose which in a more convenient description. What must be noted here, for later purposes, is that even with such mixed terms and this ambiguity thereof, we have the invariance

$$
\delta_{2} \int \mathbf{w}_{2 n-2}^{(1)}\left(\Phi_{1} ; \mathcal{A}, \mathcal{F}\right)=0
$$

and vice versa, between a pair of symmetries that are mutually commuting; $\mathcal{A}_{2}$ does not appear explicitly in $\mathbf{w}_{2 n-2}^{(1)}\left(\Phi_{1} ; \mathcal{A}, \mathcal{F}\right)$. Only $\mathcal{F}_{2}$ enters and only inside an appropriate trace.

Here we wish to explore a bit more how the anomaly descent reacts to arbitrary shifts of $\mathcal{A} \rightarrow \mathcal{A}+a$. It is useful to introduce a nilpotent BRST even operator $\Delta_{a}$, which acts on any of the above functions of $\mathcal{A}, \mathcal{F}$, and $v$ as

$$
\Delta_{a}=\mathrm{d} l_{a}+l_{a} \mathrm{~d}
$$

with the replacement $\mathrm{d} \mathcal{A} \rightarrow \mathcal{F}-\mathcal{A}^{2}$ understood. The socalled antiderivative $l_{a}$ acts as

$$
l_{a} \mathcal{A}=0, \quad l_{a} \mathcal{F}=a, \quad l_{a} v=0 .
$$

We must treat $l_{a}$ as a BRST-odd operator naturally, since $a$ is BRST odd just like $\mathcal{A}$. On the other hand, the antiderivative $l_{a}$ also obeys

$$
l_{a} \mathbf{s}+\mathbf{s} l_{a}=0
$$

for which we also need to remember that $a$, as a difference between two connections $\mathcal{A}$ and $\mathcal{A}+a$, is assumed to transform covariantly, $\mathbf{s} a=-a v-v a$.

The action of $\Delta_{a}$ on the following two quantities is of some interest:

$$
\begin{aligned}
\Delta_{a}\left(\mathbf{w}_{d+1}^{(0)}(\mathcal{A}, \mathcal{F})\right)= & \mathrm{d}\left(l_{a}\left[\mathbf{w}_{d+1}^{(0)}(\mathcal{A}, \mathcal{F})\right]\right)+l_{a}\left(P_{d+2}(\mathcal{F})\right) \\
\Delta_{a}\left(\mathbf{w}_{d}^{(1)}(v ; \mathcal{A}, \mathcal{F})\right)= & \mathrm{d}\left(l_{a}\left[\mathbf{w}_{d}^{(1)}(v ; \mathcal{A}, \mathcal{F})\right]\right) \\
& \left.+l_{a}\left(-\mathbf{s}\left[\mathbf{w}_{d+1}^{(0)}(\mathcal{A}, \mathcal{F})\right)\right]\right)
\end{aligned}
$$

Recall that, with

$$
a \cdot \mathcal{X} \equiv \int l_{a}\left[\mathbf{w}_{d+1}^{(0)}(\mathcal{A}, \mathcal{F})\right]
$$

$\mathcal{X}$ is the so-called Bardeen-Zumino current which can be added to $\mathcal{J}$ to turn the latter consistent current into its covariant version.

From this, we find

$$
\Delta_{a} \int \mathbf{w}_{d}^{(1)}(v ; \mathcal{A}, \mathcal{F})=\mathbf{s} \int l_{a}\left(\mathbf{w}_{d+1}^{(0)}\right)
$$

with a vanishing boundary condition at asymptotic infinity of the spacetime. Again, coming back to the original bosonic form,

$$
\Delta_{a} \int \mathbf{w}_{d}^{(1)}(\Phi ; \mathcal{A}, \mathcal{F})=\delta_{\Phi} \int l_{a}\left(\mathbf{w}_{d+1}^{(0)}(\mathcal{A}, \mathcal{F})\right)
$$

The integrand on the right-hand side is proportional to an inner product of $a$ with the so-called BZ current $\mathcal{X}$ as

$$
\Delta_{a} \int \mathbf{w}_{d}^{(1)}(\Phi ; \mathcal{A}, \mathcal{F})=\delta_{\Phi}(a \cdot \mathcal{X})
$$

Recall that this BZ current shifts the consistent current $\mathcal{J}$ additively into the covariant current $\mathcal{J}_{\text {cov }}=\mathcal{J}+\mathcal{X}$ [6]. 


\section{DIFFEOMORPHISM ANOMALY AND COVARIANT CURRENTS}

Let us now be more specific and split the gauge transformation into two classes; those associated with spacetime transformations, such as diffeomorphisms and supersymmetry, and the internal gauge/flavor symmetries. The notation we will adopt is

$$
\mathcal{A}=(\Gamma, \psi ; A), \quad \Phi=(\xi, \epsilon ; \vartheta), \quad \mathcal{J}=(T, S ; J),
$$

where the external gravitino $\psi$ and the supersymmetry current $S$ would be present if the theory is supersymmetric. For general discussion of the diffeomorphism anomaly and the Ward identity thereof, we will adopt the Christoffel connection $\Gamma$, although, for supersymmetry Ward identities in Sec. V, the spin connection $\omega$ is more natural and will be used instead.

We are now quite used to the fact that the variation of the effective action induces the consistent anomaly which solves the WZ consistency condition. Despite some confusions in the early literature, the relation between the consistent and the covariant anomaly was understood already in the 1980s [6]. The consistent current $\mathcal{J}$ transforms noncovariantly, but using the $\mathrm{BZ}$ term $\mathcal{X}$, which is composed entirely of the external gauge field, a covariant current can be found, namely $\mathcal{J}_{\text {cov }}=\mathcal{J}+\mathcal{X}$. The divergence of this combination is called the covariant anomaly. In turn, the covariant anomaly proved to be much more practical for computational purposes, eventually yielding the well-known anomaly polynomials [5]. The physical role of the covariant current has been much less prominent. The purpose of this section is to show that the covariant current must, in fact, appear in the Ward identity setting generically.

The message is actually very simple. The action of diffeomorphisms on the effective action should induce the consistent current of the gauge/flavor symmetry as well, since the former acts universally on all operators. On the other hand, the diffeomorphism Ward identity itself must be invariant under such internal symmetries. As we shall see, the shift to $J_{\text {cov }}=J+X$ occurs naturally. Another such symmetry transformation that acts on all currents universally is supersymmetry, which is the main subject of this paper. This section will provide a simpler illustration for the latter, more involved case, to which we will turn in Sec. IV.

\section{A. Diffeomorphism anomaly}

As a simple illustration, let us start with a theory with a gauge symmetry $\delta_{\vartheta}$ and consider how the diffeomorphism Ward identity is affected by the anomalous gauge sector. For this, let us start with

$$
\mathcal{A}=(\Gamma ; A), \quad \Phi=(\xi ; \vartheta), \quad \mathcal{J}=(T ; J),
$$

where the Christoffel connection can be conveniently elevated to a connection 1-form [6],

$$
(\Gamma)_{\beta}{ }^{\alpha} \equiv-\Gamma_{\mu \beta}^{\alpha} d x^{\mu}
$$

with the curvature 2-form

$$
R_{\beta}{ }^{\alpha}=(\mathrm{d} \Gamma+\Gamma \wedge \Gamma)_{\beta}{ }^{\alpha} .
$$

This translates to the usual Riemann curvature as

$$
\mathcal{R}_{\beta \mu \nu}^{\alpha}=\left(R_{\beta}^{\alpha}\right)_{\nu \mu} \text {. }
$$

The two are clearly the same componentwise once the symmetry properties of the Riemann tensor are invoked. Diffeomorphisms act on the Christoffel connection as

$$
\delta_{\xi} \Gamma=\mathcal{L}_{\xi}^{\prime} \Gamma+\mathrm{d}_{\Gamma}(-\partial \xi),
$$

where $\mathcal{L}_{\xi}^{\prime}$ is meant to be aware only of the 1 -form index of $\Gamma$. Note how the transformation is split into two pieces and how the second piece can be regarded as a $G L(d)$ gauge transformation with $\Phi_{\mu}{ }^{\nu}=-\partial_{\mu} \xi^{\nu}$ of $\Gamma^{3}{ }^{3}$

Another difference compared to gauge transformations is how the commutator of $\delta_{\xi}$ works. On any covariant tensor $V$, we have

$\left[\delta_{\zeta}, \delta_{\xi}\right] V=\mathcal{L}_{\xi}\left(\mathcal{L}_{\zeta} V\right)-\mathcal{L}_{\zeta}\left(\mathcal{L}_{\xi} V\right)=\mathcal{L}_{[\xi, \zeta]} V=-\delta_{[\zeta, \xi]} V$

where, as with the previous gauge variations, we let $\delta$ act on field variables only and not on parameters. This leads to the gravitational WZ consistency condition,

$$
\delta_{\zeta} \delta_{\xi} W-\delta_{\xi} \delta_{\zeta} W=\delta_{-[\zeta, \xi]} W,
$$

where one must note the unusual sign on the right-hand side. Bardeen and Zumino addressed how to solve this in their seminal paper [6] and found that the usual anomaly descent procedure solves this constraint,

$-\delta_{\xi} W=G_{\text {diffeo }}(\xi ; \Gamma, R ; F) \equiv \int \mathbf{w}_{d}^{(1)}(-\partial \xi ; \Gamma, R ; F)$,

${ }^{3}$ Its counterpart for the spin connection $\omega$ is

$$
\delta_{\xi} \omega=\mathcal{L}_{\xi}^{\prime \prime} \omega+\mathrm{d}_{\omega} \hat{\xi}_{K},
$$

where $\mathcal{L}_{\xi}^{\prime \prime}$ is again aware only of the 1 -form index of $\omega$. The $S O$ matrix $\hat{\xi}_{K}^{a b} \equiv-\nabla^{[a} \xi^{b]}-\xi^{\mu} \Omega_{\mu}^{a b}$ is known as the Kosman lift [42]. It has been established that the diffeomorphism anomaly is, modulo a counterterm, equivalent to the purely rotational $S O(d)$ Lorentz anomaly $[6,43]$. This is why we can usually get away with relying on and treating $\omega$ as if it is one of the internal gauge fields. However, the Ward identity in question is that of diffeomorphisms, for which $\Gamma$ proved to be more suitable. 
where we treat $\Gamma$ as a $G L(d)$ connection and perform the descent as if this $G L(d)$ is an internal gauge symmetry.

This can be seen from

$\delta_{\zeta} G_{\text {diffeo }}(\xi ; \Gamma, R ; F)=\int\left(\hat{\mathcal{L}}_{\zeta}^{\prime}+\delta_{-\partial \zeta}^{G L(d)}\right) \mathbf{w}_{d}^{(1)}(-\partial \xi ; \Gamma, R ; F)$,

where the hat and the prime on $\hat{\mathcal{L}}_{\zeta}^{\prime}$ mean that it acts only on fields and responds only to the differential form indices in $\Gamma$, $R$, and $F$. In particular, it perceives the entire $\mathbf{w}_{d}^{(1)}$ as a differential $d$-form. The second variation is a purely rotational $G L(d)$ gauge rotation, so one can invoke a $G L(d) \mathrm{WZ}$ consistency condition also obeyed by $G_{\text {diffeo }}(\xi ; \Gamma, R ; F)$,

$$
\begin{aligned}
& \delta_{-\partial \zeta}^{G L(d)} \int \mathbf{w}_{d}^{(1)}(-\partial \xi ; \Gamma, R ; F)-\delta_{-\partial \xi}^{G L(d)} \int \mathbf{w}_{d}^{(1)}(-\partial \xi ; \Gamma, R ; F) \\
& =\int \mathbf{w}_{d}^{(1)}([-\partial \zeta,-\partial \xi] ; \Gamma, R ; F) .
\end{aligned}
$$

On the other hand, the first derivative operator $\hat{\mathcal{L}}_{\zeta}^{\prime}$ can be made to act, by integration by parts and with the induced sign flip, on the $G L(d)$ parameter $-\partial \xi$ as if it is a (matrixvalued) scalar function.

Combining the two, we find

$$
\begin{gathered}
\delta_{\zeta} G_{\text {diffeo }}(\xi ; \Gamma, R ; F)-\delta_{\xi} G_{\text {diffeo }}(\zeta ; \Gamma, R ; F) \\
=\int \mathbf{w}_{d}^{(1)}\left(\partial\left(\zeta^{\mu} \partial_{\mu} \xi-\xi^{\mu} \partial_{\mu} \zeta\right) ; \Gamma, R ; F\right),
\end{gathered}
$$

from which

$$
\begin{aligned}
& \delta_{\zeta} G_{\text {diffeo }}(\xi ; \Gamma, R ; F)-\delta_{\xi} G_{\text {diffeo }}(\zeta ; \Gamma, R ; F) \\
& =G_{\text {diffeo }}(-[\zeta, \xi] ; \Gamma, R ; F)
\end{aligned}
$$

follows immediately, so that $-\delta_{\xi} W=G_{\text {diffeo }}(\xi ; \Gamma, R ; F)$ solves (3.9) with the obvious matrix multiplication rule. Note that in the above $G L(d)$ anomaly descent, $G_{\text {diffeo }}$ depends on $F$ but not on $A$, and only via symmetric traces of $F$, which implies that

$$
\delta_{\vartheta} G_{\text {diffeo }}(-\partial \xi ; \Gamma, R ; F)=0 .
$$

In Sec. VI, we will see how this diffeomorphism anomaly can be naturally embedded into a BRST algebra, but with a twist, which will lead us to a modification that is needed for the anomaly descent in the presence of supersymmetry as well.

\section{B. Covariant currents in the diffeomorphism Ward identity}

What about diffeomorphisms acting on the gauge anomaly? The entire anomaly is a local $d$-form integral which is linear in $\vartheta$ and polynomial in $A, F$, and $R$, all of which are tensors with respect to general coordinate transformations. Given that $\delta_{\xi}$ acts on $A, F$, and $R$ as $\mathcal{L}_{\xi}$, but does not act on $\vartheta$, we may integrate by parts to obtain

$$
\begin{aligned}
\delta_{\xi} \int \mathbf{w}_{d}^{(1)}(\vartheta ; A, F ; R) & =\int \mathbf{w}_{d}^{(1)}\left(-\mathcal{L}_{\xi} \vartheta ; A, F ; R\right) \\
& =-G\left(\mathcal{L}_{\xi} \vartheta ; A, F ; R\right) .
\end{aligned}
$$

As such, the anomaly descents that we have accumulated so far obey

$$
\begin{aligned}
& \delta_{\vartheta} G_{\text {diffeo }}(-\partial \xi ; \Gamma, R ; F)-\delta_{\xi} G(\vartheta ; A, F ; R) \\
& =G\left(\mathcal{L}_{\xi} \vartheta ; A, F ; R\right)
\end{aligned}
$$

and thus solve a consistency condition

$$
\delta_{\vartheta} \delta_{\xi} W-\delta_{\xi} \delta_{\vartheta} W=\delta_{\mathcal{L}_{\xi} \vartheta} W
$$

This last expression can be seen to be the correct WZ consistency condition as

$$
\begin{aligned}
\left(\delta_{\vartheta} \delta_{\xi}-\delta_{\vartheta} \delta_{\xi}\right) W & \left.=\left(\delta_{\vartheta}(\xi\lrcorner \mathrm{d} A+\mathrm{d}\left(\xi_{\lrcorner} A\right)\right)-\delta_{\xi}\left(\mathrm{d}_{A} \vartheta\right)\right) \cdot \frac{\delta W}{\delta A} \\
& =\left(\left(\mathrm{d} \mathcal{L}_{\xi} \vartheta\right)+\mathcal{L}_{\xi}([A, \vartheta])-\left[\mathcal{L}_{\xi} A, \vartheta\right]\right) \cdot \frac{\delta W}{\delta A} \\
& =\delta_{\mathcal{L}_{\xi} \vartheta} A \cdot \frac{\delta W}{\delta A}=\delta_{\mathcal{L}_{\xi \vartheta}} W
\end{aligned}
$$

if we keep in mind that $\delta$ 's do not act on parameters.

Here, we wish to address the transformation property of $\delta_{\xi} W$

$$
-\delta_{\xi} W=\xi_{\nu} \cdot\left\langle\nabla_{\mu} T^{\mu \nu}\right\rangle-\sum \delta_{\xi} A \cdot\langle J\rangle
$$

under $\delta_{\vartheta}$. We have seen earlier that the mixed WZ consistency condition

$$
\delta_{\vartheta}\left(\delta_{\xi} W\right)-\delta_{\xi}\left(\delta_{\vartheta} W\right)=\delta_{\mathcal{L}_{\xi \vartheta} \vartheta} W
$$

constrains $\delta_{\xi} W$, and furthermore, given the specific form of the gauge anomaly descent,

$$
\delta_{\vartheta}\left(\delta_{\xi} W\right)=0, \delta_{\xi}\left(\delta_{\vartheta} W\right)+\delta_{\mathcal{L}_{\xi} \vartheta} W=0
$$

have to hold separately. We have seen the former above, while the latter can be seen more explicitly as follows. Since $\delta_{\xi}$ is supposed to act only on field variables and not on the transformation parameters, such as $\vartheta$, we have 


$$
\begin{aligned}
\delta_{\xi}\left(\delta_{\vartheta} W\right)+\delta_{\mathcal{L}_{\xi \vartheta} \vartheta} W= & -\delta_{\xi} \int \mathbf{w}_{d}^{(1)}(\vartheta ; A ; F ; R) \\
& -\int \mathbf{w}_{d}^{(1)}\left(\mathcal{L}_{\xi} \vartheta ; A ; F ; R\right) \\
= & -\int \mathcal{L}_{\xi} \mathbf{w}_{d}^{(1)}(\vartheta ; A ; F ; R) \\
= & -\int \mathrm{d}\left(\xi_{\lrcorner} \mathbf{w}_{d}^{(1)}\right)+\xi_{\lrcorner} \mathrm{d} \mathbf{w}_{d}^{(1)}=0,
\end{aligned}
$$

where for the last equality we have used $\mathrm{dw}_{d}^{(1)}=0$ as well, which appears to contradict the formal procedure we used for the descent, but does hold once we come back to physical quantities and coordinates relevant to a $d$-dimensional spacetime; one simply cannot have a nonzero $(d+1)$-form, i.e., $\mathbf{d} \mathbf{w}_{d}^{(1)}$, in a $d$-dimensional spacetime. ${ }^{4}$

How does this reconcile with the appearance of the consistent gauge current in the Ward identity from $\delta_{\xi} W$, as opposed to the covariant one, in Eq. (3.20)? This is relatively easy to see for a single Abelian $A$. Note that $\mathcal{L}_{\xi}$ acts on $A$ as a vector

$$
\mathcal{L}_{\xi} A=\mathrm{d}\left(\xi_{\lrcorner} A\right)+\xi_{\lrcorner} \mathrm{d} A,
$$

so, with $F=\mathrm{d} A$,

$$
-\delta_{\xi} W=\xi_{\nu} \cdot\left\langle\nabla_{\mu} T^{\mu \nu}\right\rangle-\left(\xi_{\lrcorner} F\right) \cdot\langle J\rangle+\left(\xi_{\lrcorner} A\right) \cdot\left\langle\nabla_{\mu} J^{\mu}\right\rangle .
$$

On the other hand, the last piece is nothing but a pure gauge anomaly with $\vartheta=-\left(\xi_{\lrcorner} A\right)$, so it is a local functional involving $\xi, A$, and $F$. This last formula actually holds for non-Abelian $A$ as well, via $F=d A+A^{2}$.

After some tedious algebra, using the explicit form of the gauge anomaly, one realizes that this last term can be rewritten via the BZ current $X$ and the curvature $F$ such that $[24,44]$

$$
\begin{aligned}
-\delta_{\xi} W & =\xi_{\nu} \cdot\left\langle\nabla_{\mu} T^{\mu \nu}\right\rangle-\left(\xi_{\lrcorner} F\right) \cdot\langle J+X\rangle \\
& =\xi_{\nu} \cdot\left\langle\nabla_{\mu} T^{\mu \nu}\right\rangle-\left(\xi_{\lrcorner} F\right) \cdot\left\langle J_{\text {cov }}\right\rangle .
\end{aligned}
$$

As such, the diffeomorphism Ward identity is manifestly gauge invariant under $\delta_{\vartheta}$

$\xi_{\nu} \cdot\left\langle\nabla_{\mu} T^{\mu \nu}\right\rangle-\left(\xi_{\lrcorner} F\right) \cdot\left\langle J_{\mathrm{cov}}\right\rangle=\int \mathbf{w}_{d}^{(1)}(-\partial \xi ; \Gamma, R ; F)$.

The right-hand side is invariant under $\delta_{\vartheta}$ thanks to the general fact in (2.27), so the above covariantization of the gauge current is inevitable. Although the necessary conversion was performed explicitly for Abelian $A, \delta_{\vartheta}\left(\delta_{\xi} W\right)=0$ means that the same conversion of the anomaly term to the $\left(\xi_{\lrcorner} F\right) \cdot X$

\footnotetext{
${ }^{4}$ This obvious observation will also be quite useful when we introduce a generalized anomaly descent procedure for diffeomorphisms and supersymmetry in Sec. VI.
}

occurs for non-Abelian A's as well. The latter fact will appear again crucially when we discuss the supersymmetrized anomaly descent in Sec. VI.

\section{SUPERSYMMETRY AND BARDEEN-ZUMINO CURRENTS}

Let us now turn to the supersymmetry Ward identity and discuss the local fermionic terms induced by anomalies in other symmetries, which are therefore controlled by the same anomaly coefficient. Such terms induced by the gauge/flavor anomaly have been discussed long ago in [7-16,18,19], while analogous terms induced by the gravitational anomaly in two dimensions were first identified in [20,21], and locally supersymmetric contributions to gauge/favor or $R$-symmetry anomalies were discussed more recently in [24-32].

It was understood early on that, if a superspace description of the external gauge multiplet exists, such local fermionic terms can be understood as a consequence of the WZ gauge. In particular, if one keeps all auxiliary fields and maintains gauge symmetry at a fully supersymmetric level, one could move these terms to the left-hand side of the Ward identity. However, there are several contexts where a superspace or fully off-shell multiplet does not exist, and therefore we do not assume its existence in the present analysis.

As we will see, the local fermionic terms in the supersymmetry Ward identity induced by anomalies of other symmetries can be separated into two distinct types: gaugeinvariant ones and noninvariant ones [8]. In the analogous case of the diffeomorphism Ward identity, the latter was a simple consequence of the supersymmetry transformation of the external gauge fields and is completely determined by the BZ current. In the supersymmetry Ward identity, this noninvariant piece is again expressed via the $\mathrm{BZ}$ current but the way it emerges in the WZ gauge is rather different; it appears as an additional inhomogeneous contribution to the Ward identity, as demanded by the consistency condition that involves both gauge/flavor transformations and supersymmetry transformation.

The other gauge-invariant pieces, an analog of which does not exist in the diffeomorphism Ward identity, depends more sensitively on the supersymmetry algebra. What remains true, however, is that these are connected to the above noninvariant contribution again via the consistency condition. In this section, we discuss the general shape of these local fermion terms, with emphasis on the noninvariant BZ current contributions, and how exactly the same structure appears from the inflow mechanism, regardless of the precise details of the latter.

Since the noninvariant term involving the $\mathrm{BZ}$ current is entirely determined by the gauge/flavor anomaly, to begin with, one expects that this induced BZ term and the subsequent invariant pieces would be also canceled eventually if an anomaly inflow [40] cancels the gauge/flavor anomaly in question. In principle, therefore, the anomaly 
inflow may be viewed as a tool for determining all such fermionic terms, noninvariant and invariant, if the supersymmetry completion of the bulk action responsible for the inflow is known.

In general the latter is a tall order, however, given how the bulk action is typically of higher derivatives. This is true of the simplest inflow mechanism we use in this section for illustration which is itself somewhat limited, modeled after the simplest of the M5 brane inflow [45,46]. More generally, even more elaborate inflow mechanisms [47-51] are often unavoidable. Supersymmetry completion of such general topological terms in string theories or in $\mathrm{M}$ theory are hardly known.

On the other hand, if the inflow originates from a ChernSimons action in one dimension higher, the chances are better. In Sec. V, we will delve into several explicit examples of supersymmetric anomaly inflow of this kind and use them to determine the local fermionic terms in the supersymmetry Ward identity induced by anomalies in other symmetries. The Chern-Simons form $\mathbf{w}_{d+1}^{(0)}$ determines the contribution of the gauge/flavor anomaly to the supersymmetry Ward identity and plays an important role in both supersymmetric anomaly inflow and anomaly descent. A generalized anomaly descent mechanism that accommodates supersymmetry will be discussed in Sec. VI.

\section{A. Supersymmetry Ward identity revisited}

The external vector multiplet is specified by

$$
\mathcal{A}=(\psi ; A), \quad \Phi=(\epsilon ; \vartheta), \quad \mathcal{J}=(S ; J)
$$

with the gaugino superpartner $\lambda$ of $A$,

$$
\delta_{\epsilon} W=\delta_{\epsilon} A \cdot \frac{\delta W}{\delta A}+\delta_{\epsilon} \lambda \cdot \frac{\delta W}{\delta \lambda} .
$$

As in the case of diffeomorphisms above, we wish to explore what consequences follow from the WZ consistency condition,

$$
\delta_{\vartheta}\left(\delta_{\epsilon} W\right)-\delta_{\epsilon}\left(\delta_{\vartheta} W\right)=0 .
$$

The second term on the left is

$$
-\delta_{\epsilon}\left(\delta_{\vartheta} W\right)=\delta_{\epsilon} \int \mathbf{w}_{d}^{(1)}(\vartheta ; A, F) .
$$

Recalling the algebra that leads to the BZ current $X$,

$$
\Delta_{\delta_{\epsilon} A} \int \mathbf{w}_{d}^{(1)}(\vartheta ; A, F)=\delta_{\vartheta} \int l_{\delta_{\epsilon} A}\left(\mathbf{w}_{d+1}^{(0)}(A, F)\right),
$$

where $l_{\delta_{\epsilon} A}$ is the antiderivative we reviewed earlier, we realize that

$$
-\delta_{\epsilon}\left(\delta_{\vartheta} W\right)=\delta_{\vartheta}\left(\delta_{\epsilon} A \cdot X\right),
$$

again with the BZ current $X$ of the gauge symmetry. This means that, even in the absence of true anomaly term $G(\epsilon ; \psi ; A, \lambda), \delta_{\epsilon} W$ cannot vanish, but rather must obey

$$
-\delta_{\epsilon} W=\delta_{\epsilon} A \cdot X+\cdots
$$

with the ellipsis denoting terms that are invariant under $\delta_{\vartheta}$ and are determined by the consistency condition following from two successive supersymmetry transformations, namely

$$
\left.\left(\delta_{\epsilon} \delta_{\epsilon^{\prime}}-\delta_{\epsilon^{\prime}} \delta_{\epsilon}\right) W=\left(\delta_{\xi}+\delta_{\Lambda}\right) W, \quad \xi^{\mu} \sim \bar{\epsilon}^{\prime} \gamma^{\mu} \epsilon, \Lambda \sim \xi\right\lrcorner A .
$$

Shortly we will offer an alternate method for this supersymmetry completion via anomaly inflow, so here we mostly focus on the term involving the BZ current.

An interesting fact is that we can rewrite this Ward identity as [24]

$$
\epsilon \cdot\left\langle\nabla_{\mu} S^{\mu}\right\rangle-\delta_{\epsilon} A \cdot(\langle J\rangle+X)-\cdots=0,
$$

where we have moved the BZ current and its supersymmetry completion, meaning additional gauge-invariant terms whose presence is demanded by the WZ consistency condition and the very first BZ piece, to the left-hand side. Once this shift of $J$ to $J_{\text {cov }}=J+X$ is done, each component of this Ward identity is individually $\delta_{\vartheta}$ invariant. Again we see that $J_{\text {cov }}$ appears naturally but the way this happens here is a little different from the diffeomorphisms case, where it happened via a rearrangement of the consistent current term.

Here, the main message is that this shift is something to be expected once we realize that in the Ward identities of spacetime symmetries the gauge/flavor current appears always in its covariant form. This also means that the shift is immediate and explicitly known once the gauge anomaly is known, without having to compute an entirely new set of diagrams; the shift by the BZ current $X$ is determined entirely by the $\delta_{\vartheta}$ anomaly.

The local fermionic terms, $\delta_{\epsilon} A \cdot X+\cdots$, in the supersymmetry Ward identity should be distinguished from what one might consider an inherent anomaly of supersymmetry. Nevertheless, this shift of the Ward identity is not devoid of physical consequences and must be kept track of carefully; for example, it has known consequences in various supersymmetric partition function computations that rely on curved spacetime background and various external fluxes.

In superspace, or in terms of a fully off-shell external vector multiplet, if such a description exists, the covariant current and its accompanying supersymmetry partners would have appeared naturally on the left-hand side, on par with the diffeomorphism example of the previous section. This would allow one to say that the effective 
action is invariant under supersymmetry transformations [8]. However, one should be mindful that such a fully off-shell extension is not always available. Even if one is available, as would be the case with a smaller supersymmetry content, it is not clear what one would gain in practice by avoiding the WZ gauge choice. We might as well concentrate on the nature of these anomalous contributions to the Ward identity; they would not disappear even in the superspace version, but merely be attributed differently, and, as has been seen recently $[24,36]$, can produce a tangible difference in some localization computations.

\section{B. Symmetry restoration via gauge/flavor anomaly inflow}

The simplest form of anomaly inflow can be found from a topological coupling of the type

$$
S_{\text {inflow }}=(-1)^{p} \int_{X_{D}} H_{p+1} \wedge \mathbf{w}_{d+1}^{(0)}(\mathcal{A})
$$

in an ambient spacetime $X_{D}$ with $p=D-d-2$. A $d$-dimensional world volume enters the picture as a magnetic defect to the spacetime gauge field strength $H_{p+1}=\mathrm{d} A_{p}+\cdots$ such that

$$
\mathrm{d} H_{p+1}=\delta_{M_{d}}
$$

and supports an anomalous field content. Suppose that we have an effective action $W(\mathcal{A})$ on $M_{d}$ whose anomaly polynomial is $P_{d+2}=\mathrm{d} \mathbf{w}_{d+1}^{(0)}(\mathcal{A})$.

A gauge variation of $S_{\text {inflow }}$ leads to

$(-1)^{p} \int_{X_{D}} H_{p+1} \wedge \delta_{\Phi} \mathbf{w}_{d+1}^{(0)}(\mathcal{A})=\int_{M_{d}} \mathbf{w}_{d}^{(1)}(\Phi ; \mathcal{A})$,

so that the combined effective action

$$
\mathcal{W}(\mathcal{A})=W(\mathcal{A})+S_{\text {inflow }}
$$

is gauge invariant, $\delta_{\Phi} \mathcal{W}=0$. While one must consider more involved versions of this to cover all known types of anomaly inflow, the essence of the inflow mechanism is well represented by the example above.

Here, let us ask a slightly different question, namely whether $\delta_{\epsilon} \mathcal{W}$ is gauge invariant. Recall that the $\mathrm{WZ}$ consistency condition demands that we have

$$
-\delta_{\epsilon} W=\delta_{\epsilon} A \cdot X+\cdots,
$$

where the BZ current $X$ can be traced back to the gauge anomaly $\delta_{\vartheta} W \neq 0$. If the latter is canceled by the anomaly inflow from $S_{\text {inflow }}$, it is only natural to expect that $\delta_{\epsilon} A \cdot X$ on the right-hand side of the supersymmetry variation is also canceled as well, so that the quantity $\delta_{\epsilon} \mathcal{W}$ is gauge invariant.
The relevant quantity to compute is ${ }^{5}$

$$
\left((-1)^{p} \int_{X_{D}} H_{p+1} \wedge \Delta_{\delta_{\epsilon} A} \mathbf{w}_{d+1}^{(0)}(\mathcal{A})\right)
$$

Using the descent mechanism we have learned in the previous sections, this becomes

$$
\begin{aligned}
& (-1)^{p} \int_{X_{D}} H_{p+1} \wedge\left(\mathrm{d} l_{\delta_{\epsilon} A}+l_{\delta_{\epsilon} A} \mathrm{~d}\right)\left(\mathbf{w}_{d+1}^{(0)}(\mathcal{A})\right) \\
& =(-1)^{p} \int_{X_{D}} H_{p+1} \wedge\left(\mathrm{d}\left[\delta_{\epsilon} A \wedge X\right]+l_{\delta_{\epsilon} A} P_{d+2}(\mathcal{F})\right) .
\end{aligned}
$$

The first term, when integrated by parts, reduces to

$$
\delta_{\epsilon} A \cdot X
$$

Combining the two contributions, we find

$$
\delta_{\epsilon} \mathcal{W}=\delta_{\epsilon} W+\delta_{\epsilon} S_{\text {inflow }}=0+\cdots,
$$

where the ellipsis denotes all terms we have neglected so far, namely the gauge-invariant part of $\delta_{\epsilon} W$ and the second piece of (4.16), which does not naturally reduce to the world volume. Therefore, we learn that, modulo the bulk piece in $\delta_{\epsilon} S_{\text {inflow }}$, the gauge invariance of $\delta \mathcal{W}$ is restored through the gauge anomaly inflow, such that

$$
\delta_{\vartheta}\left(\delta_{\epsilon} \mathcal{W}\right)=0
$$

on the world volume.

So, what about the bulk pieces in the variation of $S_{\text {inflow }}$ ? Once we embed these discussions to supersymmetric theories in the bulk, we should expect any leftover bulk terms, such as the second piece in (4.16) to be canceled by the transformation of the superpartners. As such, in order to complete the study, we need to start from the fully supersymmetrized form of $\delta_{\epsilon} W$ and $S_{\text {inflow }}$. For minimal rigid supersymmetry the former is known from, e.g., [8-10] for up to $d=6$, off shell and in the WZ gauge, while the latter could prove more involved. After all, such topological terms, $S_{\text {inflow }}$, involve generically higher derivative terms and were often discovered only via the anomaly cancellation of the entire string theory or M theory.

On the other hand, there are simpler subclasses where this kind of question can be asked. One is when the inflow is achieved by a Green-Schwarz mechanism with $D=d$ and another is where

\footnotetext{
${ }^{5}$ We think of the world volume spinors as a chiral projection of spacetime spinors on $X_{D}$. Some specific examples of such embedding are discussed in Sec. V.
} 


$$
S_{\text {inflow }}=\int_{X_{d+1}} \mathbf{w}_{d+1}^{(0)}(A)+\cdots
$$

on a bulk $X_{d+1}$ whose boundary is the world volume $M_{d}$. In fact, the entire class of anti-de Sitter (AdS) $)_{d+1}$ theories coupled to boundary conformal field theory belong to such a class. With the proper supersymmetry instituted in these $S_{\text {inflow }}$, one could ask what happens to the supersymmetry variation of the combined action $\delta_{\epsilon} \mathcal{W}$.

We will see in the next section, example by example, that once all these terms are delineated, we find that not only gauge invariance is restored

$$
\delta_{\Phi}\left(\delta_{\epsilon} \mathcal{W}\right)=0
$$

with $\mathcal{W}=W+S_{\text {inflow }}$, but the combined action is fully supersymmetric,

$$
\delta_{\epsilon} \mathcal{W}=0
$$

Thus, let us call this mechanism of anomaly cancellation a "supersymmetric anomaly inflow."

\section{SUPERSYMMETRIC ANOMALY INFLOW}

In the previous section we considered supersymmetric theories with an anomaly

$$
-\delta_{\vartheta} W=\int \mathbf{w}_{d}^{(1)}(\Phi ; \mathcal{A}, \mathcal{F}),
$$

for which the supersymmetry Ward identity acquires unexpected terms on the right-hand side,

$$
-\delta_{\epsilon} W=\int l_{\delta_{\epsilon} \mathcal{A}} \mathbf{w}_{d+1}^{(0)}(\mathcal{A}, \mathcal{F})+\cdots
$$

with the usual anomaly descent relating the two right-hand sides as $\delta_{\vartheta} \mathbf{w}_{d+1}^{(0)}=\mathrm{d} \mathbf{w}_{d}^{(1)}$. Although we have discussed this explicitly for gauge/flavor symmetry here, a similar structure arises for $R$-symmetry and diffeomorphism anomalies, which we will revisit in later sections.

On the other hand, given a canceling anomaly inflow term $S_{\text {inflow }}$, we saw that the combined effective action $\mathcal{W}=W+S_{\text {inflow }}$ satisfies

$$
\delta_{\Phi} \mathcal{W}=0, \quad \delta_{\Phi}\left(\delta_{\epsilon} \mathcal{W}\right)=0 .
$$

Given that the two right-hand sides of $\delta W$ above originate from a common gauge anomaly, it is quite natural to expect that

$$
\delta_{\epsilon} \mathcal{W}=0,
$$

as long as the inflow action is properly supersymmetrized. In this section, we demonstrate this mechanism for several examples where a supersymmetric $S_{\text {inflow }}$ is available.

Note that this means that there are alternate ways to compute the right-hand side of (5.2), without having to solve directly the WZ consistency conditions. In a sense, such an alternate computation via $S_{\text {inflow }}$ can be regarded as a supersymmetrized anomaly descent especially when $S_{\text {inflow }}=S_{\mathrm{CS}}$. This is not to say that one can always achieve the same kind of universal procedure as in the usual anomaly inflow, since the crucial supersymmetrization of $S_{\mathrm{CS}}$ is often cumbersome. One complication is that, with a physical inflow mechanism, given the typically higher dimensional nature of $S_{\text {inflow }}$, both supersymmetry and the field content thereof tends to be enlarged, beyond what is strictly necessary for $W$. In the next section we explore a more streamlined and mathematically compact version of the supersymmetric anomaly descent, inspired by these inflow phenomena. As we show at the end of that section, however, the two approaches coincide for certain multiplets, with the descent procedure determining the supersymmetric Chern-Simons form implementing the codimension-one inflow.

In the concrete examples of supersymmetric anomaly inflow that we present in the remaining of this section, we wish to emphasize the utility of such a mechanism as an efficient way to determine the form of supersymmetrized anomalies. To this end, we flip the sign of the ChernSimons terms relative to the discussion above, $S_{\text {inflow }}=$ $-S_{\mathrm{CS}}$, in the following such that

$$
\delta_{\epsilon} W=\delta_{\epsilon} S_{\mathrm{CS}}
$$

\section{A. Gauge/flavor anomaly inflow}

The first example of a supersymmetric anomaly inflow we consider is in the context of flavor anomalies in $d$ dimensions. The anomaly inflow mechanism we focus on relies on the existence of a suitable supersymmetric ChernSimons action in $d+1$ dimensions. Higher codimension inflow may also be possible in certain cases, such as on the world volume of D-branes, but we will not consider this mechanism here.

A supersymmetric Chern-Simons action for a given gauge multiplet in $d+1$ dimensions determines the supersymmetric flavor anomaly in $d$ dimensions for any theory that can consistently couple to the background gauge multiplet obtained from that in $d+1$ dimensions by dimensional reduction. The flavor anomalies for all multiplets with less supersymmetry can be obtained by consistently truncating the resulting $d$-dimensional multiplet.

However, given a theory with $\mathcal{N}$-extended supersymmetry in $d$ dimensions, there may not exist a corresponding Chern-Simons action with the same amount of supersymmetry in $d+1$ dimensions. In such cases, the $d$-dimensional 
gauge multiplet must first be embedded in one with a larger amount of supersymmetry, for which a supersymmetric Chern-Simons action does exist. This situation arises, for example, for theories with $(p, q)$ supersymmetry in two dimensions when $p \neq q$. In order to cancel the anomaly through anomaly inflow in such cases, the $(p, q)$ multiplet must be embedded in the closest nonchiral one, namely the flavor multiplet with $\left(p^{\prime}, p^{\prime}\right)$ supersymmetry, where $p^{\prime}=\max (p, q)$. As long as $p^{\prime} \leq 3$, there exists an $\mathcal{N}=p^{\prime}$ supersymmetric Chern-Simons action that provides an anomaly inflow mechanism for the $\left(p^{\prime}, p^{\prime}\right)$ anomaly in two dimensions. The original $(p, q)$ anomaly is obtained by multiplet truncation.

\section{1. $3 D$ to $2 D$ anomaly inflow}

The simplest examples of supersymmetric anomaly inflow arise for $d=2$, since the relevant Chern-Simons actions in three dimensions are typically known. The most general 3D Chern-Simons action without matter multiplets involves the $\mathcal{N}=3$ vector multiplet [52,53], which consists of a gauge field $A_{\mu}$, three Majorana gauginos $\lambda^{I}, I=1$, 2,3 , three real scalars $\sigma^{I}$, and real auxiliary scalars $D^{I}$, as well as a Majorana fermion $\chi$. All fields are in the adjoint representation of the gauge group. ${ }^{6}$ As we now show, the $\mathcal{N}=3$ supersymmetric Chern-Simons action provides a supersymmetric inflow mechanism for the flavor anomaly of the $\mathcal{N}=(3,3)$ vector multiplet in two dimensions and hence for any $\mathcal{N}=(p, q)$ vector multiplet with $p, q \leq 3$.

We should clarify at this point that the flavor anomalies for nonchiral theories, such as $\mathcal{N}=(3,3)$ theories in $2 \mathrm{D}$ or $\mathcal{N}=2$ ones in $4 \mathrm{D}$ that we consider below, are somewhat formal and discussed only as nontrivial solutions of the WZ consistency conditions. Their coefficients vanish in all Lagrangian theories, as well as in non-Lagrangian theories obtained through renormalization group flows from Lagrangian ones, due to 't Hooft anomaly matching. More relevant to the present discussion, however, is the fact that such anomalies for nonchiral multiplets appear as an intermediate step for the computation of supersymmetrized flavor anomalies in chiral theories via anomaly inflow.

The supersymmetry transformations of the $\mathcal{N}=3$ vector multiplet are parametrized by three real Majorana spinors $\epsilon^{I}$ and are given by

$$
\begin{aligned}
\delta_{Q}(\epsilon) \sigma^{I} & =\varepsilon^{I}{ }_{J K} \bar{\epsilon}^{J} \lambda^{K}-\bar{\epsilon}^{I} \chi, \\
\delta_{Q}(\epsilon) A_{\mu} & =\bar{\epsilon}^{I} \gamma_{\mu} \lambda^{I}, \\
\delta_{Q}(\epsilon) \lambda_{\alpha}^{I} & =\frac{1}{2}\left(\gamma^{\mu \nu} \epsilon^{I}\right)_{\alpha} F_{\mu \nu}+\varepsilon^{I}{ }_{J K} \epsilon_{\alpha}^{J} D^{K}+\varepsilon^{I}{ }_{J K}\left(\gamma^{\mu} \epsilon^{J}\right)_{\alpha} \mathcal{D}_{\mu} \sigma^{K}-i\left[\sigma^{I}, \sigma_{J}\right] \epsilon_{\alpha}^{J}, \\
\delta_{Q}(\epsilon) \chi_{\alpha} & =-\epsilon_{\alpha}^{I} D_{I}+\left(\gamma^{\mu} \epsilon^{I}\right)_{\alpha} \mathcal{D}_{\mu} \sigma_{I}-\frac{i}{2} \varepsilon_{I J K}\left[\sigma^{J}, \sigma^{K}\right] \epsilon_{\alpha}^{I}, \\
\delta_{Q}(\epsilon) D^{I} & =\varepsilon^{I}{ }_{J K} \bar{\epsilon}^{J} \gamma^{\mu} \mathcal{D}_{\mu} \lambda^{K}+\bar{\epsilon}^{I} \gamma^{\mu} \mathcal{D}_{\mu} \chi-i\left[\bar{\epsilon}^{J} \lambda_{J}, \sigma^{I}\right]+i\left[\bar{\epsilon}^{I} \lambda^{J}+\bar{\epsilon}^{J} \lambda^{I}, \sigma_{J}\right]-i \varepsilon^{I}{ }_{J K}\left[\bar{\epsilon}^{J} \chi, \sigma^{K}\right],
\end{aligned}
$$

where we follow the spinor conventions of [54] and we have suppressed the gauge group indices. The gaugecovariant derivative and field strength are respectively

$$
\begin{aligned}
\mathcal{D}_{\mu} & =\partial_{\mu}-i\left[A_{\mu},\right], \\
F_{\mu \nu} & =\partial_{\mu} A_{\nu}-\partial_{\nu} A_{\mu}-i\left[A_{\mu}, A_{\nu}\right] .
\end{aligned}
$$

Moreover, the indices $I, J, K$ are raised and lowered with the Kronecker delta $\delta^{I J}, \delta_{I J}$. Notice that $\varepsilon^{I J K}=\varepsilon_{I J K}$ denotes the Levi-Civita symbol in $\mathbb{R}^{3}$ spanned by the $I, J, K$ indices, while $\varepsilon^{\mu \nu \rho}$ is the Levi-Civita symbol in $\mathbb{R}^{1,2}$.

The $\mathcal{N}=2$ and $\mathcal{N}=1$ vector multiplets can be obtained from the $\mathcal{N}=3$ one by setting specific

\footnotetext{
${ }^{6}$ In this section we use Hermitian generators for the gauge group, while we find it convenient to formulate the anomaly descent in terms of anti-Hermitian generators. The two choices are related as $t_{\mathrm{H} .}^{a}=i t_{\mathrm{a} . \mathrm{H} .}^{a}$.
}

components to zero. The resulting nonzero components for these multiplets are

\begin{tabular}{ccc} 
& SUSY parameters & Nonzero components \\
\hline $\mathcal{N}=2$ & $\epsilon^{1}, \epsilon^{2}$ & $A_{\mu}, \sigma_{3}, D_{3}, \lambda_{1}, \lambda_{2}$ \\
$\mathcal{N}=1$ & $\epsilon^{1}$ & $A_{\mu}, \lambda_{1}$ \\
\hline
\end{tabular}

It is straightforward to check that these are consistent truncations of the off-shell supersymmetry (SUSY) transformations (5.6).

Using the $3 \mathrm{D}$ identities

$$
\begin{array}{r}
\delta_{\left[K^{I} \varepsilon_{L]}{ }^{P Q}+\delta^{I[P} \varepsilon^{Q]}{ }_{K L}\right.}=0, \\
\delta_{[K}^{I} \varepsilon_{L]}^{P Q}+\delta_{[K}^{P} \varepsilon_{L]}^{Q I}+\delta_{[K}^{Q} \varepsilon_{L]}^{I P}=0,
\end{array}
$$

one can show that, together with rigid translations $\delta_{D}(\xi)=$ $\xi^{\mu} \partial_{\mu}$ and gauge transformations, which act on the vector multiplet fields as $\delta_{G}(\vartheta) A_{\mu}=\mathcal{D}_{\mu} \vartheta$, and $\delta_{G}(\vartheta)=i[\vartheta$, for all other fields, the transformations (5.6) close off shell and obey the algebra 


$$
\left[\delta_{Q}\left(\epsilon_{1}\right), \delta_{Q}\left(\epsilon_{2}\right)\right]=\delta_{D}(\xi)+\delta_{G}(\vartheta)
$$

with the composite translation and gauge parameters given respectively by

$$
\xi^{\mu}=2 \bar{\epsilon}_{2}^{I} \gamma^{\mu} \epsilon_{1 I}, \quad \vartheta=-\xi^{\mu} A_{\mu}-2 \varepsilon_{I J K}\left(\bar{\epsilon}_{2}^{I} \epsilon_{1}^{J}\right) \sigma^{K} .
$$

Notice that $\xi^{\mu}$ is constant (as required) while $\vartheta$ is not, due to the explicit field dependence.

The $\mathcal{N}=3$ supersymmetric Chern-Simons Lagrangian is [53]

$$
\begin{aligned}
\mathcal{L}_{\mathrm{CS}}= & \frac{k}{4 \pi} \operatorname{tr}\left(\kappa \varepsilon^{\mu \nu \rho}\left(A_{\mu} \partial_{\nu} A_{\rho}-\frac{2 i}{3} A_{\mu} A_{\nu} A_{\rho}\right)-\bar{\lambda}^{I} \lambda_{I}\right. \\
& \left.+\bar{\chi} \chi-2 \sigma^{I} D_{I}+\frac{i}{3} \varepsilon_{I J K} \sigma^{I}\left[\sigma^{J}, \sigma^{K}\right]\right)
\end{aligned}
$$

where $k$ is the Chern-Simons level, while $\kappa= \pm 1$ parametrizes a choice in the 3D Clifford algebra through the identity

$$
\gamma^{\mu \nu}=-\kappa \varepsilon^{\mu \nu \rho} \gamma_{\rho}, \quad \kappa= \pm 1 .
$$

A generic variation of the Chern-Simons Lagrangian (5.12) takes the form

$$
\begin{aligned}
\delta \mathcal{L}_{\mathrm{CS}}= & \frac{k}{4 \pi} \operatorname{tr}\left(\kappa \varepsilon^{\mu \nu \rho}\left(\partial_{\rho}\left(A_{\nu} \delta A_{\mu}\right)+\delta A_{\mu} F_{\nu \rho}\right)-2 \bar{\lambda}^{I} \delta \lambda_{I}\right. \\
& \left.+2 \bar{\chi} \delta \chi-2 \sigma^{I} \delta D_{I}-\delta \sigma^{I}\left(2 D_{I}-i \varepsilon_{I J K}\left[\sigma^{J}, \sigma^{K}\right]\right)\right) .
\end{aligned}
$$

Specializing this variation to gauge and $\mathcal{N}=3$ supersymmetry transformations, we find that in both cases (5.12) is invariant up to a total derivative term, namely

$$
\begin{aligned}
\delta_{G}(\vartheta) \mathcal{L}_{\mathrm{CS}}= & \frac{k}{4 \pi} \kappa \varepsilon^{\mu \nu \rho} \partial_{\mu} \operatorname{tr}\left(\vartheta \partial_{\nu} A_{\rho}\right), \\
\delta_{Q}(\epsilon) \mathcal{L}_{\mathrm{CS}}= & \frac{k}{4 \pi} \partial_{\mu} \operatorname{tr}\left(\kappa \varepsilon^{\mu \nu \rho} \delta_{Q}(\epsilon) A_{\nu} A_{\rho}\right. \\
& \left.-2 \sigma^{I}\left(\varepsilon_{I J K} \bar{\epsilon}^{J} \gamma^{\mu} \lambda^{K}+\bar{\epsilon}_{I} \gamma^{\mu} \chi\right)\right) .
\end{aligned}
$$

The boundary term resulting from a gauge transformation matches the usual non-Abelian anomaly, i.e., the bosonic part of the 2D flavor anomaly. As we now discuss, the boundary term arising from supersymmetry transformations corresponds to its $(3,3)$ supersymmetric completion, i.e., the $(3,3)$ supersymmetry completion of the anomaly in the WZ gauge, which follows from the WZ consistency conditions.

Upon dimensional reduction to two dimensions, the 3D $\mathcal{N}=3$ vector multiplet reduces to the $2 \mathrm{D} \mathcal{N}=(3,3)$ vector multiplet (see, e.g., [55] for the Abelian case). This has the same field content as the $3 \mathrm{D} \mathcal{N}=3$ vector multiplet, except that the $3 \mathrm{D}$ gauge field gives rise to an extra scalar: $A_{\mu}=\left(A_{\hat{\mu}}, \phi\right)$, where $\hat{\mu}=0,1$. The supersymmetry transformations of the $2 \mathrm{D} \mathcal{N}=(3,3)$ vector multiplet follow from the $3 \mathrm{D} \mathcal{N}=3$ transformations in (5.6), namely

$$
\begin{aligned}
\delta_{Q}(\epsilon) \sigma^{I}= & \varepsilon^{I}{ }_{J K} \bar{\epsilon}^{J} \lambda^{K}-\bar{\epsilon}^{I} \chi \\
\delta_{Q}(\epsilon) A_{\hat{\mu}}= & \bar{\epsilon}^{I} \gamma_{\hat{\mu}} \lambda^{I}, \\
\delta_{Q}(\epsilon) \phi= & \kappa \bar{\epsilon}^{I} \gamma_{*} \lambda^{I}, \\
\delta_{Q}(\epsilon) \lambda_{\alpha}^{I}= & \frac{1}{2}\left(\gamma^{\hat{\mu} \hat{\omega}} \epsilon^{I}\right)_{\alpha} F_{\hat{\mu} \hat{\nu}}+\kappa\left(\gamma^{\hat{\mu}} \gamma_{*} \epsilon^{I}\right)_{\alpha} \mathcal{D}_{\hat{\mu}} \phi+\varepsilon_{J K}^{I} \epsilon_{\alpha}^{J} D^{K}+\varepsilon_{J K}^{I}\left(\gamma^{\hat{\mu}} \epsilon^{J}\right)_{\alpha} \mathcal{D}_{\hat{\mu}} \sigma^{K} \\
& -i \kappa \varepsilon_{J K}^{I}\left(\gamma_{*} \epsilon^{J}\right)_{\alpha}\left[\phi, \sigma^{K}\right]-i\left[\sigma^{I}, \sigma_{J}\right] \epsilon_{\alpha}^{J}, \\
\delta_{Q}(\epsilon) \chi_{\alpha}= & -\epsilon_{\alpha}^{I} D_{I}+\left(\gamma^{\hat{\mu}} \epsilon^{I}\right)_{\alpha} \mathcal{D}_{\hat{\mu}} \sigma_{I}-i \kappa\left(\gamma_{*} \epsilon^{I}\right)_{\alpha}\left[\phi, \sigma_{I}\right]-\frac{i}{2} \varepsilon_{I J K}\left[\sigma^{J}, \sigma^{K}\right] \epsilon_{\alpha}^{I}, \\
\delta_{Q}(\epsilon) D^{I}= & \varepsilon^{I}{ }_{J K} \bar{\epsilon}^{J} \gamma^{\hat{\mu}} \mathcal{D}_{\hat{\mu}} \lambda^{K}-i \kappa \varepsilon_{J K}^{I} \bar{\epsilon}^{J} \gamma_{*}\left[\phi, \lambda^{K}\right]+\bar{\epsilon}^{I} \gamma^{\hat{\mu}} \mathcal{D}_{\hat{\mu}} \chi-i \kappa \bar{\epsilon}^{I} \gamma_{*}[\phi, \chi] \\
& -i\left[\bar{\epsilon}^{J} \lambda_{J}, \sigma^{I}\right]+i\left[\bar{\epsilon}^{I} \lambda^{J}+\bar{\epsilon}^{J} \lambda^{I}, \sigma_{J}\right]-i \varepsilon_{J K}^{I}\left[\bar{\epsilon}^{J} \chi, \sigma^{K}\right],
\end{aligned}
$$

where $\gamma_{*} \equiv \kappa \gamma^{2}=\kappa \gamma_{2}$ is the chirality matrix in two dimensions (also denoted by $\gamma_{3}$; see [54]). These satisfy the algebra (5.10) with $2 \mathrm{D}$ parameters

$\xi^{\hat{\mu}}=2 \bar{\epsilon}_{2}^{I} \gamma^{\hat{\mu}} \epsilon_{1 I}, \quad \vartheta=-\xi^{\hat{\mu}} A_{\hat{\mu}}-2 \kappa \bar{\epsilon}_{2}^{I} \gamma_{3} \epsilon_{1 I} \phi-2 \varepsilon_{I J K}\left(\bar{\epsilon}_{2}^{I} \epsilon_{1}^{J}\right) \sigma^{K}$.
When expressed in terms of the $2 \mathrm{D}$ vector multiplet, the symmetry variations (5.15) of the $\mathcal{N}=3$ Chern-Simons action provide - by construction - a solution of the WZ consistency conditions for the 2D $(3,3)$ symmetry algebra. We therefore conclude that the $(3,3)$ flavor anomaly in two dimensions takes the form 


$$
\begin{aligned}
\delta_{G}(\vartheta) W= & \frac{k}{4 \pi} \varepsilon^{\hat{\nu} \hat{\rho}} \int d^{2} x \operatorname{tr}\left(\vartheta \partial_{\hat{\nu}} A_{\hat{\rho}}\right), \\
\delta_{Q}(\epsilon) W= & \frac{k}{4 \pi} \int d^{2} x \operatorname{tr}\left(\varepsilon^{\hat{\nu} \hat{\rho}} \delta_{Q}(\epsilon) A_{\hat{\nu}} A_{\hat{\rho}}\right. \\
& \left.-2 \kappa \sigma^{I}\left(\varepsilon_{I J K} \bar{\epsilon}^{J} \gamma_{*} \lambda^{K}+\bar{\epsilon}_{I} \gamma_{*} \chi\right)\right),
\end{aligned}
$$

where $\varepsilon_{(2)}^{\hat{\mu} \hat{\nu}} \equiv \kappa \varepsilon_{(3)}^{2 \hat{\mu} \hat{\nu}}$. This generalizes known results for $2 \mathrm{D}$ gauge anomalies for theories with less supersymmetry $[10,12,14]$. The $(p, q)$ flavor anomaly for any $p, q \leq 3$ can be obtained from the $(3,3)$ anomaly in $(5.18)$ by a suitable truncation of the vector multiplet.

\section{5D to $4 D$ anomaly inflow}

$\mathcal{N} \leq 2$ flavor symmetry in four dimensions presents another example of supersymmetric anomaly inflow. The $\mathcal{N}=1$ off-shell gauge multiplet in five dimensions $[54,56-59]^{7}$ comprises a gauge field $A_{\mu}$, a symplectic Majorana spinor $\lambda^{i}$ that transforms as a doublet of the $S U$ (2) $R$-symmetry group, a real scalar $\sigma$, and an auxiliary real symmetric tensor $Y^{i j}=Y^{j i}$, all in the adjoint representation of the gauge group. Following again the conventions of [54], the supersymmetry transformations are

$$
\begin{aligned}
\delta_{Q}(\epsilon) A_{\mu}^{a} & =\frac{1}{2} \bar{\epsilon}^{i} \gamma_{\mu} \lambda_{i}^{a}, \quad \delta_{Q}(\epsilon) \sigma^{a}=\frac{i}{2} \bar{\epsilon}^{i} \lambda_{i}^{a}, \\
\delta_{Q}(\epsilon) Y^{i j a} & =-\frac{1}{2} \bar{\epsilon}^{(i}\left(\mathcal{D} \lambda^{j) a}+i f_{b c}{ }^{a} \sigma^{b} \lambda^{j) c}\right), \\
\delta_{Q}(\epsilon) \lambda^{i a} & =-\frac{1}{4} \gamma^{\mu \nu} F_{\mu \nu}^{a} \epsilon^{i}-\frac{i}{2} \mathcal{D} \sigma^{a} \epsilon^{i}-Y^{i j a} \epsilon_{j},
\end{aligned}
$$

where the covariant derivative and field strength of the gauge field are given by

$$
\mathcal{D}_{\mu} \phi^{a} \equiv\left(\mathcal{D}_{\mu} \phi\right)^{a}=\partial_{\mu} \phi^{a}+f_{b c}{ }^{a} A_{\mu}^{b} \phi^{c}, \quad \phi^{a}=\text { any field },
$$

$$
F_{\mu \nu}^{a}=\partial_{\mu} A_{\nu}^{a}-\partial_{\nu} A_{\mu}^{a}+f_{b c}{ }^{a} A_{\mu}^{b} A_{\nu}^{c} .
$$

Together with rigid translations, $\delta_{D}(\xi)=\xi^{\mu} \partial_{\mu}$ and gauge transformations that act as $\delta_{G}(\vartheta) A_{\mu}^{a}=\left(\mathcal{D}_{\mu} \vartheta\right)^{a}=\partial_{\mu} \vartheta^{a}+$ $f_{b c}{ }^{a} A_{\mu}^{b} \vartheta^{c}$ and $\delta_{G}(\vartheta) \phi^{a}=f_{b c}{ }^{a} \phi^{b} \vartheta^{c}$ on all other components of the multiplet, the supersymmetry transformations (5.19) satisfy the algebra

$$
\begin{aligned}
{\left[\delta_{Q}\left(\epsilon_{1}\right), \delta_{Q}\left(\epsilon_{2}\right)\right] } & =\delta_{D}(\xi)+\delta_{G}(\vartheta), \quad \xi^{\mu}=\frac{1}{2} \bar{\epsilon}_{2}^{i} \gamma^{\mu} \epsilon_{1 i}, \\
\vartheta^{a} & =-\xi^{\mu} A_{\mu}^{a}-\frac{i}{2} \bar{\epsilon}_{2}^{i} \epsilon_{1 i} \sigma^{a} .
\end{aligned}
$$

An important subtlety in five dimensions is that there exists no pure Chern-Simons action for the vector multiplet. $\mathcal{N}=2$ supersymmetric Lagrangians are specified by a prepotential $\mathcal{F}(\sigma)$, which couples the Chern-Simons and Yang-Mills parts of the action. Although a pure Yang-Mills Lagrangian is obtained from a quadratic prepotential, a supersymmetric Chern-Simons Lagrangian requires a cubic prepotential and necessarily contains a Yang-Mills part. In particular, the supersymmetric Chern-Simons Lagrangian takes the form

$$
\begin{aligned}
\mathcal{L}_{\mathrm{CS}}= & \left(-\frac{1}{4} F_{\mu \nu}^{a} F^{b \mu \nu}-\frac{1}{2} \bar{\lambda}^{i a} \mathcal{D} \lambda_{i}^{b}-\frac{1}{2} \mathcal{D}_{\mu} \sigma^{a} \mathcal{D}^{\mu} \sigma^{b}+Y_{i j}^{a} Y^{i j b}\right) \mathcal{F}_{a b} \\
& +\left(\frac{\kappa}{24} \varepsilon^{\mu \nu \rho \sigma \tau} A_{\mu}^{a}\left(F_{\nu \rho}^{b} F_{\sigma \tau}^{c}+f_{d e}{ }^{b} A_{\nu}^{d} A_{\rho}^{e}\left(-\frac{1}{2} F_{\sigma \tau}^{c}+\frac{1}{10} f_{f g}^{c} A_{\sigma}^{f} A_{\tau}^{g}\right)\right)\right. \\
& \left.-\frac{i}{8} \bar{\lambda}^{i a} \gamma^{\mu \nu} F_{\mu \nu}^{b} \lambda_{i}^{c}-\frac{i}{2} \bar{\lambda}^{i a} \lambda^{j b} Y_{i j}^{c}+\frac{i}{4} f_{d e}^{c} \sigma^{a} \sigma^{b} \bar{\lambda}^{i d} \lambda_{i}^{e}\right) \mathcal{F}_{a b c},
\end{aligned}
$$

where again $\kappa= \pm 1$ parametrizes a choice in the representation of the Clifford algebra in five dimensions through the relation $\gamma^{\mu \nu \rho \sigma \tau}=-i \kappa \varepsilon^{\mu \nu \rho \sigma \tau}$. Moreover, $\mathcal{F}_{a b}, \mathcal{F}_{a b c}$ denote respectively the second and third derivatives of the prepotential $\mathcal{F}$, which we take to be

\footnotetext{
${ }^{7}$ To avoid cluttering the notation we use $\mu, \nu, \rho, \ldots$ to denote both $4 \mathrm{D}$ and $5 \mathrm{D}$ spacetime indices, since the distinction should be clear from the context.
}

$$
\mathcal{F}(\sigma)=\frac{k}{48 \pi^{2}} d_{a b c} \sigma^{a} \sigma^{b} \sigma^{c},
$$

where $d_{a b c}=\operatorname{tr}\left(t_{a}\left\{t_{b}, t_{c}\right\}\right)$ is the completely symmetric rank-3 invariant tensor on the Lie algebra of the gauge group and $k$ is the gauge/flavor anomaly coefficient that depends on the microscopic theory.

The prepotential (5.24) is chosen such that the gauge transformation of the Chern-Simons action (5.23) coincides with the bosonic part of the consistent gauge/flavor anomaly in four dimensions upon the identification $\varepsilon_{(4)}^{\mu \nu \rho \sigma} \equiv \kappa \varepsilon_{(5)}^{4 \mu \nu \rho \sigma}$, namely 


$$
\begin{aligned}
\delta_{G}(\vartheta) \mathcal{L}_{\mathrm{CS}}= & \frac{k}{48 \pi^{2}} \int d^{4} x d_{a b c} \vartheta^{a} \varepsilon^{\mu \nu \rho \sigma} \partial_{\mu} \\
& \times\left(A_{\nu}^{b} \partial_{\rho} A_{\sigma}^{c}+\frac{1}{4} f_{d e}^{c} A_{\nu}^{b} A_{\rho}^{d} A_{\sigma}^{e}\right) .
\end{aligned}
$$

The supersymmetry transformation of the Chern-Simons Lagrangian (5.23) is also a total derivative, which we determine next. By construction, the two symmetry transformations of the 5D supersymmetric Chern-Simons action provide a solution of the WZ consistency conditions for the
$\mathcal{N}=2$ symmetry algebra in four dimensions and therefore determine the $\mathcal{N}=2$ supersymmetric flavor/anomaly.

A lengthy computation using several identities for $5 \mathrm{D}$ symplectic Majorana spinors (see Appendix A of [57]) and the Lie algebra relation

$$
d_{c(a b} f_{e) d^{c}}=0
$$

determines that the supersymmetry variation of (5.23) takes the form

$$
\begin{aligned}
\delta_{Q}(\epsilon) \mathcal{L}_{\mathrm{CS}}= & \partial_{\mu}\left[\left(-\frac{1}{4}\left(\bar{\epsilon}^{i} \gamma_{\nu} \lambda_{i}^{a}\right) F^{b \mu \nu}-\frac{i}{4}\left(\bar{\epsilon}^{i} \lambda_{i}^{a}\right) \mathcal{D}^{\mu} \sigma^{b}-\frac{1}{2}\left(\bar{\epsilon}_{i} \gamma^{\mu} \lambda_{j}^{a}\right) Y^{i j b}\right.\right. \\
& \left.-\frac{1}{8}\left(\bar{\epsilon}^{i} \gamma^{\mu \rho \sigma} \lambda_{i}^{a}\right) F_{\rho \sigma}^{b}-\frac{i}{4}\left(\bar{\epsilon}^{i} \gamma^{\mu \nu} \lambda_{i}^{a}\right) \mathcal{D}_{\nu} \sigma^{b}\right) \mathcal{F}_{a b} \\
& +\frac{\kappa}{6} \varepsilon^{\mu \nu \rho \sigma \tau}\left(\delta_{Q}(\epsilon) A_{\nu}^{a}\left(2 A_{\rho}^{b} \partial_{\sigma} A_{\tau}^{c}+\frac{3}{4} f_{d e}^{b} A_{\rho}^{c} A_{\sigma}^{d} A_{\tau}^{e}+\frac{1}{8} \bar{\lambda}^{i b} \gamma_{\rho \sigma \tau} \lambda_{i}^{c}\right)\right. \\
& \left.\left.+\frac{1}{32}\left(\bar{\epsilon}^{i} \gamma_{\nu \rho} \lambda_{i}^{a}\right)\left(\bar{\lambda}^{j b} \gamma_{\sigma \tau} \lambda_{j}^{c}\right)\right) \mathcal{F}_{a b c}\right] .
\end{aligned}
$$

Once expressed in terms of the $4 \mathrm{D} \mathcal{N}=2$ multiplet fields arising from the dimensional reduction of the $5 \mathrm{D} \mathcal{N}=1$ vector multiplet, this variation coincides with the supersymmetric completion of the $\mathcal{N}=2$ gauge/flavor anomaly in four dimensions.

The $4 \mathrm{D} \mathcal{N}=2$ vector multiplet possesses the same field content as the corresponding 5D multiplet, except that the components $A_{4}^{a}$ of the 5D gauge field combine with the scalars $\sigma^{a}$ into a complex scalar: $X^{a}=\frac{1}{2}\left(A_{4}^{a}-i \sigma^{a}\right)$. Moreover, the 5D symplectic Majorana gaugino reduces to an $S U(2)$ doublet of either chiral or Majorana gauginos in four dimensions. Following [54], we decompose the 5D gaugino and supersymmetry parameter in terms of chiral spinors in four dimensions as (see Appendix 20.B in [54])

$$
\begin{gathered}
\lambda_{i}^{a}=-\lambda_{(4)}^{j a} \varepsilon_{j i}+\lambda_{(4) i}^{a}, \quad \bar{\lambda}_{i}^{a}=\bar{\lambda}_{(4)}^{j a} \varepsilon_{j i}+\bar{\lambda}_{(4) i}^{a}, \quad \lambda_{(4) i}^{a}=P_{L} \lambda_{(4) i}^{a}, \quad \lambda_{(4)}^{i a}=P_{R} \lambda_{(4)}^{i a}, \\
\epsilon_{i}=\epsilon_{(4)}^{j} \varepsilon_{j i}+\epsilon_{(4) i}, \quad \bar{\epsilon}_{i}=\bar{\epsilon}_{(4)}^{j} \varepsilon_{j i}-\bar{\epsilon}_{(4) i}, \quad \epsilon_{(4) i}=P_{R} \epsilon_{(4) i}, \quad \epsilon_{(4)}^{i}=P_{L} \epsilon_{(4)}^{i},
\end{gathered}
$$

where $P_{L}=\frac{1}{2}\left(1-\kappa \gamma_{*}\right)$ and $P_{R}=\frac{1}{2}\left(1+\kappa \gamma_{*}\right)$ are the $4 \mathrm{D}$ chirality projectors and $\gamma_{*}=-\kappa \gamma^{4}$. In particular, $\epsilon_{(4)}^{i}, \epsilon_{(4) i}$ and $\lambda_{(4)}^{i a}$, $\lambda_{(4) i}^{a}$ are charge conjugate pairs so that $\epsilon_{(4)}^{i}+\epsilon_{(4) i}$ and $\lambda_{(4)}^{i a}+\lambda_{(4) i}^{a}$ are Majorana.

Inserting this decomposition in (5.19) and dropping the subscript (4) leads to the $4 \mathrm{D} \mathcal{N}=2$ supersymmetry transformations

$$
\begin{aligned}
\delta_{Q}(\epsilon) X^{a} & =\frac{1}{2} \bar{\epsilon}^{i} \lambda_{i}^{a}, \\
\delta_{Q}(\epsilon) \lambda_{i}^{a} & =\mathcal{D} X^{a} \epsilon_{i}+\frac{1}{4} \gamma^{\mu \nu} F_{\mu \nu}^{a} \varepsilon_{i j} \epsilon^{j}+Y_{i j}^{a} \epsilon^{j}+X^{b} \bar{X}^{c} f_{b c}{ }^{a} \varepsilon_{i j} \epsilon^{j}, \\
\delta_{Q}(\epsilon) A_{\mu}^{a} & =\frac{1}{2} \varepsilon^{i j} \bar{\epsilon}_{i} \gamma_{\mu} \lambda_{j}^{a}+\frac{1}{2} \varepsilon_{i j} \bar{\epsilon}^{i} \gamma_{\mu} \lambda^{j a}, \\
\delta_{Q}(\epsilon) Y^{i j a} & =\frac{1}{2} \bar{\epsilon}^{(i} \mathcal{D} \lambda^{j) a}+f_{b c}{ }^{a} X^{b} \varepsilon^{(i k} \bar{\epsilon}_{k} \lambda^{j) c}+\frac{1}{2} \varepsilon^{(i k} \varepsilon^{j) l} \bar{\epsilon}_{k} \mathcal{D} \lambda_{l}^{a}-f_{b c}{ }^{a} \bar{X}^{b} \bar{\epsilon}^{(i} \lambda_{l}^{c} \varepsilon^{j) l},
\end{aligned}
$$

where now $\mu, \nu=0,1,2,3$. Together with gauge transformations and translations, these satisfy the same algebra as in five dimensions, but with composite parameters 


$$
\xi^{\mu}=\frac{1}{2} \bar{\epsilon}_{2}^{i} \gamma^{\mu} \epsilon_{1 i}+\text { H.c., } \quad \vartheta^{a}=-\xi^{\mu} A_{\mu}^{a}+X^{a} \varepsilon^{i j} \bar{\epsilon}_{2 i} \epsilon_{1 j}+\bar{X}^{a} \varepsilon_{i j} \bar{\epsilon}_{2}^{i} \epsilon_{1}^{j} .
$$

We can now evaluate the symmetry transformations (5.25) and (5.27) of the 5D Chern-Simons Lagrangian in terms of the 4D $\mathcal{N}=2$ vector multiplet components in order to obtain the $\mathcal{N}=2$ supersymmetric gauge/flavor anomaly in four dimensions,

$$
\begin{aligned}
\delta_{G}(\vartheta) W= & \frac{k}{48 \pi^{2}} \int d^{4} x d_{a b c} \vartheta^{a} \varepsilon^{\mu \nu \rho \sigma} \partial_{\mu}\left(A_{\nu}^{b} \partial_{\rho} A_{\sigma}^{c}+\frac{1}{4} f_{d e}{ }^{c} A_{\nu}^{b} A_{\rho}^{d} A_{\sigma}^{e}\right), \\
\delta_{Q}(\epsilon) W= & \frac{k}{16 \pi^{2}} \int d^{4} x d_{a b c}\left[i ( X ^ { c } - \overline { X } ^ { c } ) \left(\varepsilon_{i j}\left(\bar{\epsilon}^{i} \gamma^{\nu} \lambda^{j a}\right) \mathcal{D}_{\nu} X^{b}+\varepsilon^{i j}\left(\bar{\epsilon}_{i} \gamma^{\nu} \lambda_{j}^{a}\right) \mathcal{D}_{\nu} \bar{X}^{b}\right.\right. \\
& \left.-\left(\bar{\epsilon}^{i} \lambda_{i}^{a}-\bar{\epsilon}_{i} \lambda^{i a}\right) f_{d e}{ }^{b} X^{d} \bar{X}^{e}+\left(\bar{\epsilon}^{j} \lambda_{i}^{a}+\bar{\epsilon}_{i} \lambda^{j a}\right) Y_{j}^{i b}-\frac{1}{4}\left(\bar{\epsilon}^{i} \gamma^{\rho \sigma} \lambda_{i}^{a}+\bar{\epsilon}_{i} \gamma^{\rho \sigma} \lambda^{i a}\right) F_{\rho \sigma}^{b}\right) \\
& +\frac{1}{3} \varepsilon^{\nu \rho \sigma \tau}\left(\delta_{Q}(\epsilon) A_{\nu}^{a}\left(2 A_{\rho}^{b} \partial_{\sigma} A_{\tau}^{c}+\frac{3}{4} f_{d e}^{b} A_{\rho}^{c} A_{\sigma}^{d} A_{\tau}^{e}+\frac{1}{8} \bar{\lambda}^{i b} \gamma_{\rho \sigma \tau} \lambda_{i}^{c}+\frac{1}{8} \bar{\lambda}_{i}^{b} \gamma_{\rho \sigma \tau} \lambda^{i c}\right)\right. \\
& \left.\left.+\frac{1}{32}\left(\bar{\epsilon}^{i} \gamma_{\nu \rho} \lambda_{i}^{a}-\bar{\epsilon}_{i} \gamma_{\nu \rho} \lambda^{i a}\right)\left(\varepsilon_{k l} \bar{\lambda}^{k b} \gamma_{\sigma \tau} \lambda^{l c}+\varepsilon^{k l} \bar{\lambda}_{k}^{b} \gamma_{\sigma \tau} \lambda_{l}^{c}\right)\right)\right] .
\end{aligned}
$$

This generalizes well-known results for the $\mathcal{N}=1$ gauge/flavor anomaly, which we can easily recover from (5.31) through a truncation of the $\mathcal{N}=2$ multiplet.

The $4 \mathrm{D} \mathcal{N}=1$ vector multiplet with a Majorana gaugino and the corresponding supersymmetry transformations can be obtained from the $\mathcal{N}=2$ multiplet by setting

$$
\begin{aligned}
\epsilon_{1} & =\epsilon^{1}=0, & \lambda_{2}^{a}=\lambda^{2 a}=0, & X^{a}=0, \quad Y^{11 a}=Y^{22 a}=0, \\
\epsilon & \equiv \epsilon_{2}+\epsilon^{2}, & \lambda^{a} \equiv \lambda_{1}^{a}+\lambda^{1 a}, & D^{a} \equiv-2 i \kappa Y^{12 a} .
\end{aligned}
$$

Inserting these in (5.29) results in the $\mathcal{N}=1$ supersymmetry transformations

$$
\delta_{Q}(\epsilon) A_{\mu}^{a}=-\frac{1}{2} \bar{\epsilon} \gamma_{\mu} \lambda^{a}, \quad \delta_{Q}(\epsilon) \lambda^{a}=\left(\frac{1}{4} \gamma^{\mu \nu} F_{\mu \nu}^{a}+\frac{i}{2} \gamma_{*} D^{a}\right) \epsilon, \quad \delta_{Q}(\epsilon) D^{a}=\frac{i}{2} \bar{\epsilon} \gamma_{*} \gamma^{\mu} \mathcal{D}_{\mu} \lambda^{a},
$$

which satisfy the algebra (the subscripts 1 and 2 here should not to be confused with the $S U(2)$ indices of the $\mathcal{N}=2$ multiplet)

$$
\left[\delta_{Q}\left(\epsilon_{1}\right), \delta_{Q}\left(\epsilon_{2}\right)\right]=\delta_{D}\left(\frac{1}{2} \bar{\epsilon}_{2} \gamma^{\mu} \epsilon_{1}\right)+\delta_{G}\left(-\frac{1}{2} \bar{\epsilon}_{2} \gamma^{\mu} \epsilon_{1} A_{\mu}\right)
$$

Evaluating the transformations (5.31) on the truncated multiplet (5.32) results in the $\mathcal{N}=1$ supersymmetric gauge/flavor anomaly in four dimensions [8-10]

$$
\begin{aligned}
& \delta_{G}(\vartheta) W=\frac{k}{48 \pi^{2}} \int d^{4} x d_{a b c} \vartheta^{a} \varepsilon^{\mu \nu \rho \sigma} \partial_{\mu}\left(A_{\nu}^{b} \partial_{\rho} A_{\sigma}^{c}+\frac{1}{4} f_{d e}^{c} A_{\nu}^{b} A_{\rho}^{d} A_{\sigma}^{e}\right), \\
& \delta_{Q}(\epsilon) W=\frac{k}{48 \pi^{2}} \int d^{4} x d_{a b c} \varepsilon^{\mu \nu \rho \sigma} \delta_{Q}(\epsilon) A_{\mu}^{a}\left(2 A_{\nu}^{b} \partial_{\rho} A_{\sigma}^{c}+\frac{3}{4} f_{d e}^{b} A_{\nu}^{c} A_{\rho}^{d} A_{\sigma}^{e}+\frac{1}{8} \bar{\lambda}^{b} \gamma_{\nu \rho \sigma} \lambda^{c}\right) .
\end{aligned}
$$

\section{B. Current multiplet anomaly inflow}

We now turn to anomaly inflow for local supersymmetry, which forms an algebra with diffeomorphisms, local Lorentz transformations, and $R$ symmetry. The gauge fields corresponding to these local symmetries comprise an off-shell supergravity multiplet, which couples minimally to the current multiplet containing the stress tensor, supercurrent, and $\mathrm{R}$ current. 
TABLE I. The field content of the off-shell $\mathcal{N}=6$ conformal supergravity field content. All $S O(6)$ indices $I, J, K=1, \ldots, 6$ are totally antisymmetrized. Notice that all components are neutral under the $U(1)$ factor of the $R$-symmetry group.

\begin{tabular}{lcccccccc}
\hline \hline Field & $e_{\mu}{ }^{a}$ & $\psi_{\mu}^{I}$ & $B_{\mu}^{I J}$ & $B_{\mu}$ & $\lambda^{I J K}$ & $\lambda^{I}$ & $E^{I J}$ & $D^{I J}$ \\
\hline Weyl weight & 1 & $\frac{1}{2}$ & 0 & 0 & $-\frac{3}{2}$ & $-\frac{3}{2}$ & -1 & -2 \\
$S U(4)$ representation & 1 & 6 & 15 & 1 & 20 & 6 & 15 & 15 \\
$U(1)$ charge & 0 & 0 & 0 & 0 & 0 & 0 & 0 & 0 \\
\hline \hline
\end{tabular}

For a fixed amount of supersymmetry in a given spacetime dimension there exist different off-shell supergravity multiplets that differ in auxiliary field content and the symmetries they gauge. The gravity multiplet with the minimal auxiliary field content is off-shell conformal supergravity that gauges the entire superconformal group and includes Weyl and S-supersymmetry transformations. Any other off-shell supergravity multiplet with the same amount of supersymmetry can be obtained by coupling specific matter multiplets to conformal supergravity and imposing suitable gauge-fixing conditions. This results in a larger field content relative to conformal supergravity, but less symmetries due to the partial gauge-fixing conditions. For example, old minimal [60-62], new minimal [63-66], and $16+16$ [67-69] supergravities in four dimensions can all be obtained in this way from $\mathcal{N}=1$ conformal supergravity [70-73]. These couple respectively to the Ferrara-Zumino, R-, and S-current multiplets [74], while conformal supergravity couples to the conformal current multiplet.

Gravitational and Lorentz anomalies exist in $d=4 k+2$ dimensions and can be obtained from a Chern-Simons action in $d=4 k+3$ dimensions [5,6]. Like flavor anomalies, gravitational and Lorentz anomalies result in an associated Q-supersymmetrized anomaly, which contributes a local term to the divergence of the supercurrent $[10,20,21]$. For the minimal $\mathcal{N}=(1,0)$ supergravity in two dimensions, the supersymmetric completion of the gravitational anomaly has also been shown to follow from a supersymmetric gravitational Chern-Simons action in three dimensions [20]. Another local contribution to the divergence of the supercurrent arises in all even dimensions in the presence of an $R$-symmetry anomaly [24,26-28,30,31]. The $R$-symmetry anomaly can be obtained from a Chern-Simons action in $d+1$ dimensions too, and one expects that its supersymmetric completion should follow similarly from a supersymmetric Chern-Simons action.

In this subsection we discuss the gravitational/Lorentz anomaly of the $(p, q)$ conformal current multiplet in two dimensions and, in particular, all mixed anomalies it generates. As we will see, there are two effects that control the structure of these anomalies. First, local supersymmetry requires that the gravitational/Lorentz and $R$-symmetry anomalies be considered in tandem, since the underlying algebra mixes the corresponding symmetries. Second, both
TABLE II. Consistent truncations of the $\mathcal{N}=6$ Weyl multiplet.

\begin{tabular}{ll}
\hline \hline & Nonzero components \\
\hline $\mathcal{N}=5$ & $e_{\mu}{ }^{a}, \psi_{\mu}^{I}, B_{\mu}^{I J}, \lambda^{I J K}, \lambda^{6}, E^{I 6}, D^{I 6}$ \\
$\mathcal{N}=4$ & $e_{\mu}{ }^{a}, \psi_{\mu}^{I}, B_{\mu}^{I J}, \lambda^{I J K}, E^{56}, D^{56}$ \\
$\mathcal{N}=3$ & $e_{\mu}{ }^{a}, \psi_{\mu}^{I}, B_{\mu}^{I J}, \lambda^{123}$ \\
$\mathcal{N}=2$ & $e_{\mu}^{a}, \psi_{\mu}^{I}, B_{\mu}^{12}$ \\
$\mathcal{N}=1$ & $e_{\mu}{ }^{a}, \psi_{\mu}^{1}$ \\
\hline \hline
\end{tabular}

the gravitational/Lorentz and $R$-symmetry anomalies produce mixed anomalies for all other symmetries of the multiplet. Our goal here is to obtain all these anomalies through an inflow mechanism from an off-shell supergravity Chern-Simons action in three dimensions [75-83].

As a side comment, we note that all anomalies of the $(p, q)$ conformal current multiplet in two dimensions may alternatively be obtained holographically from the ChernSimons action of the (on-shell) $(p, q) \mathrm{AdS}_{3}$ supergravity of Achucarro and Townsend $[84,85]$, which gauges the supergroup $\operatorname{OSp}(p \mid 2 ; \mathbb{R}) \times \operatorname{OSp}(q \mid 2 ; \mathbb{R}){ }^{8}$. Such a holographic calculation would reproduce not only the gravitational/ Lorentz anomaly and the resulting mixed anomalies $[87,88]$, but also the Weyl anomaly [89] and its supersymmetric completion [24,25]. However, here we are interested specifically in obtaining the current multiplet anomalies through anomaly inflow, and so we focus on the gravitational/Lorentz anomaly.

\section{3D to $2 D$ anomaly inflow}

The maximal off-shell conformal supergravity (Weyl) multiplet is the $\mathcal{N}=8$ multiplet [77,78], but a ChernSimons action is known only for up to $\mathcal{N}=6[82,83]$. The $R$-symmetry group of these multiplets is $S O(\mathcal{N})$ with the exception of the $\mathcal{N}=6$ multiplet, in which case it is enhanced to $S O(6) \times U(1) \cong U(4)$. Upon dimensional reduction to two dimensions, the $3 \mathrm{D}$ gravity multiplets reduce to $(\mathcal{N}, \mathcal{N})$ off-shell conformal supergravity in two dimensions, which gauges the $\operatorname{OSp}(\mathcal{N} \mid 2 ; \mathbb{R}) \times$ $O S p(\mathcal{N} \mid 2 ; \mathbb{R})$ conformal group. A peculiarity of $2 \mathrm{D}$ supergravity is that a single $S O(\mathcal{N})$ gauge field gauges both left and right copies of the $S O(\mathcal{N})_{L} \times S O(\mathcal{N})_{R} R$ symmetry [90].

We follow the component formulation of [82] and focus on the $\mathcal{N}=6$ Weyl multiplet since it is the maximal one for which a Chern-Simons action is known. All multiplets with less supersymmetry can be obtained by suitable truncations, as shown in Table II. The field content of the off-shell $\mathcal{N}=6$ Weyl multiplet consists of the dreibein $e_{\mu}{ }^{a}$, six Majorana gravitinos $\psi_{\mu}^{I}, \quad I=1, \ldots, 6$, and the $S O(6) \times U(1)$ gauge fields, respectively $B_{\mu}^{I J}$ and $B_{\mu}$, as well as two sets of auxiliary Majorana spinors $\lambda^{I J K}$ and $\lambda^{I}$, and two sets of auxiliary scalars $E^{I J}$ and $D^{I J}$. The $S O(6)$ indices

\footnotetext{
${ }^{8}$ See [86] for an early special case of such a derivation.
} 
$I, J, K=1, \ldots, 6$ in all fields are totally antisymmetrized. The Weyl weight and $R$-symmetry representation of all components are given in Table I.

The local symmetries of the $\mathcal{N}=6$ Weyl multiplet comprise diffeomorphisms, parametrized by the infinitesimal vector field $\xi^{\mu}(x)$, local Lorentz transformations, $\lambda^{a b}(x)=-\lambda^{b a}(x)$, Weyl rescalings, $\sigma(x), S O(6) \times U(1) R$-symmetry transformations parametrized by $\theta^{I J}(x)=-\theta^{J I}(x)$ and $\theta(x)$, as well as Q- and S-supersymmetry transformations, parametrized respectively by the local Grassmann-valued Majorana parameters $\epsilon^{I}(x)$ and $\eta^{I}(x)$. The infinitesimal transformations of all fields under the bosonic symmetries take the form

$$
\begin{aligned}
\delta_{B} e_{\mu}{ }^{a} & =\xi^{\nu} \partial_{\nu} e_{\mu}{ }^{a}+\partial_{\mu} \xi^{\nu} e_{\nu}{ }^{a}-\lambda^{a}{ }_{b} e_{\mu}{ }^{b}+\sigma e_{\mu}{ }^{a}, \\
\delta_{B} \psi_{\mu}^{I} & =\xi^{\nu} \partial_{\nu} \psi_{\mu}^{I}+\partial_{\mu} \xi^{\nu} \psi_{\nu}^{I}-\frac{1}{4} \lambda_{a b} \gamma^{a b} \psi_{\mu}^{I}+\frac{1}{2} \sigma \psi_{\mu}^{I}-\theta^{I J} \psi_{\mu}^{J}, \\
\delta_{B} B_{\mu}^{I J} & =\xi^{\nu} \partial_{\nu} B_{\mu}^{I J}+\partial_{\mu} \xi^{\nu} B_{\nu}^{I J}+\partial_{\mu} \theta^{I J}+B^{I K} \theta_{\mu}^{K J}+B^{J K} \theta_{\mu}^{I K}, \\
\delta_{B} B_{\mu} & =\xi^{\nu} \partial_{\nu} B_{\mu}+\partial_{\mu} \xi^{\nu} B_{\nu}+\partial_{\mu} \theta, \\
\delta_{B} \lambda^{I J K} & =\xi^{\nu} \partial_{\nu} \lambda^{I J K}-\frac{1}{4} \lambda_{a b} \gamma^{a b} \lambda^{I J K}-\frac{3}{2} \sigma \lambda^{I J K}-\theta^{I L} \lambda^{L J K}-\theta^{J L} \lambda^{I L K}-\theta^{K L} \lambda^{I J L}, \\
\delta_{B} \lambda^{I} & =\xi^{\nu} \partial_{\nu} \lambda^{I}-\frac{1}{4} \lambda_{a b} \gamma^{a b} \lambda^{I}-\frac{3}{2} \sigma \lambda^{I}-\theta^{I L} \lambda^{L J K}, \\
\delta_{B} E^{I J} & =\xi^{\nu} \partial_{\nu} E^{I J}-\sigma E^{I J}-\theta^{I K} E^{K J}-\theta^{J K} E^{I K}, \\
\delta_{B} D^{I J} & =\xi^{\nu} \partial_{\nu} D^{I J}-2 \sigma D^{I J}-\theta^{I K} D^{K J}-\theta^{J K} D^{I K},
\end{aligned}
$$

where we have used the shorthand notation $\delta_{B} \equiv \delta_{D}(\xi)+\delta_{L}(\lambda)+\delta_{W}(\sigma)+\delta_{R}(\theta)$.

Under Q supersymmetry the components of the Weyl multiplet transform as

$$
\begin{aligned}
\delta_{Q}(\epsilon) e_{\mu}{ }^{a} & =\frac{1}{4} \bar{\epsilon}^{I} \gamma^{a} \psi_{\mu}^{I}, \quad \delta_{Q}(\epsilon) \psi_{\mu}^{I}=\mathcal{D}_{\mu} \epsilon^{I}, \\
\delta_{Q}(\epsilon) B_{\mu}^{I J} & =-\bar{\epsilon}^{[I} \psi_{\mu+}^{J]}+\frac{1}{2 \sqrt{2}} \bar{\epsilon}^{K} \gamma_{\mu} \lambda^{I J K}+\frac{1}{4 \sqrt{2}} \varepsilon^{I J K L M N} \bar{\epsilon}^{K} \psi_{\mu}^{L} E^{M N}, \\
\delta_{Q}(\epsilon) B_{\mu} & =\frac{1}{2 \sqrt{2}} \bar{\epsilon}^{I} \gamma_{\mu} \lambda^{I}-\frac{1}{2 \sqrt{2}} \bar{\epsilon}^{I} \psi_{\mu}^{J} E^{I J}, \\
\delta_{Q}(\epsilon) \lambda^{I J K} & =-\frac{3}{4 \sqrt{2}} \gamma^{\mu \nu} \epsilon^{[I} \hat{G}_{\mu \nu}^{J K]}+\frac{1}{2} \varepsilon^{I J K L M N} \epsilon^{L} D^{M N}+\frac{1}{4} \varepsilon^{I J K L M N} \gamma^{\mu} \epsilon^{L} \hat{\mathcal{D}}_{\mu} E^{M N}-\frac{3}{\sqrt{2}} \epsilon^{L} E^{[I J} E^{K L]}, \\
\delta_{Q}(\epsilon) \lambda^{I} & =-\frac{1}{4 \sqrt{2}} \gamma^{\mu \nu} \epsilon^{I} \hat{G}_{\mu \nu}+\epsilon^{J} D^{I J}-\frac{1}{2} \gamma^{\mu} \epsilon^{J} \hat{\mathcal{D}}_{\mu} E^{I J}+\frac{1}{8 \sqrt{2}} \varepsilon^{I J K L M N} \epsilon^{J} E^{K L} E^{M N}, \\
\delta_{Q}(\epsilon) E^{I J} & =\frac{1}{2} \bar{\epsilon}^{[I} \lambda^{J]}-\frac{1}{24} \varepsilon^{I J K L M N} \bar{\epsilon}^{K} \lambda^{L M N}, \\
\delta_{Q}(\epsilon) D^{I J} & =\frac{1}{2} \bar{\epsilon}^{[I} \lambda_{+}^{J]}+\frac{1}{24} \varepsilon^{I J K L M N} \bar{\epsilon}^{K} \lambda_{+}^{L M N},
\end{aligned}
$$

while S supersymmetry acts as

$$
\begin{array}{rlrl}
\delta_{S}(\eta) e_{\mu}{ }^{a} & =0, & \delta_{S}(\eta) \psi_{\mu}^{I}=\gamma_{\mu} \eta^{I}, & \delta_{S}(\eta) B_{\mu}^{I J}=\frac{1}{2} \bar{\eta}^{[I} \psi_{\mu}^{J]}, \\
\delta_{S}(\eta) B_{\mu} & =0, & \delta_{S}(\eta) \lambda^{I J K}=-\frac{1}{2} \varepsilon^{I J K L M N} \eta^{L} E^{M N}, & \delta_{S}(\eta) \lambda^{I}=\eta^{J} E^{I J}, \\
\delta_{S}(\eta) E^{I J}=0, & \delta_{S}(\eta) D^{I J}=-\frac{1}{4} \bar{\eta}^{[I} \lambda^{J]}-\frac{1}{48} \varepsilon^{I J K L M N} \bar{\eta}^{K} \lambda^{L M N} .
\end{array}
$$

Following [82], we have introduced the abbreviations 


$$
\begin{aligned}
\psi_{\mu+}^{I} & =\frac{1}{4} \gamma^{\rho \sigma} \gamma_{\mu} \psi_{\rho \sigma}^{I}, \quad \psi_{\mu \nu}^{I}=\mathcal{D}_{[\mu} \psi_{\nu]}^{I}, \\
\lambda_{+}^{I J K} & =-\frac{1}{2} \gamma^{\mu} \hat{\mathcal{D}}_{\mu} \lambda^{I J K}+\frac{1}{2} \varepsilon^{I J K L M N} \gamma^{\mu} \psi_{\mu+}^{L} E^{M N}-\frac{3}{4 \sqrt{2}} \varepsilon^{M N P Q[I J} \lambda^{K] M N} E^{P Q}+\frac{3}{\sqrt{2}} \lambda^{[I} E^{J K]}, \\
\lambda_{+}^{I} & =-\frac{1}{2} \gamma^{\mu} \hat{\mathcal{D}}_{\mu} \lambda^{I}-\gamma^{\mu} \psi_{\mu+}^{J} E^{I J}+\frac{1}{2 \sqrt{2}} \lambda^{I J K} E^{J K}, \\
\hat{G}_{\mu \nu}^{I J} & =G_{\mu \nu}^{I J}+2 \bar{\psi}_{[\mu}^{[I} \psi_{\nu]+}^{J]}-\frac{1}{\sqrt{2}} \bar{\psi}_{[\mu}^{K} \gamma_{\nu} \lambda^{I J K}-\frac{1}{4 \sqrt{2}} \varepsilon^{I J K L M N} \bar{\psi}_{\mu}^{K} \psi_{\nu}^{L} E^{M N}, \\
\hat{G}_{\mu \nu} & =G_{\mu \nu}-\frac{1}{\sqrt{2}} \bar{\psi}_{[\mu}^{I} \gamma_{\nu]} \lambda^{I}+\frac{1}{2 \sqrt{2}} \bar{\psi}_{\mu}^{I} \psi_{\nu}^{J} E^{I J}, \\
\hat{\mathcal{D}}_{\mu} E^{I J} & =\mathcal{D}_{\mu} E^{I J}-\frac{1}{2} \bar{\psi}_{\mu}^{[I} \lambda^{J]}+\frac{1}{24} \varepsilon^{I J K L M N} \bar{\psi}_{\mu}^{K} \lambda^{L M N}, \\
\hat{\mathcal{D}}_{\mu} \lambda^{I J K} & =\mathcal{D}_{\mu} \lambda^{I J K}+\frac{3}{4 \sqrt{2}} \gamma^{\rho \sigma} \psi_{\mu}^{[I} \hat{G}_{\rho \sigma}^{I K]}-\frac{1}{2} \varepsilon^{I J K L M N} \psi_{\mu}^{L} D^{M N}-\frac{1}{4} \varepsilon^{I J K L M N} \gamma^{\rho} \psi_{\mu}^{L} \hat{\mathcal{D}}_{\rho} E^{M N}+\frac{3}{\sqrt{2}} \psi_{\mu}^{L} E^{[I J} E^{K L]}, \\
\hat{\mathcal{D}}_{\mu} \lambda^{I} & =\mathcal{D}_{\mu} \lambda^{I}+\frac{1}{4 \sqrt{2}} \gamma^{\rho \sigma} \psi_{\mu}^{I} \hat{G}_{\rho \sigma}-\psi_{\mu}^{J} D^{I J}+\frac{1}{2} \gamma^{\rho} \psi_{\mu}^{J} \hat{\mathcal{D}}_{\rho} E^{I J}-\frac{1}{8 \sqrt{2}} \varepsilon^{I J K L M N} \psi_{\mu}^{J} E^{K L} E^{M N},
\end{aligned}
$$

where $G_{\mu \nu}^{I J}$ and $G_{\mu \nu}$ denote the field strengths of the $R$-symmetry gauge fields

$$
\begin{aligned}
G_{\mu \nu}^{I J} & =\partial_{\mu} B_{\nu}^{I J}-\partial_{\nu} B_{\mu}^{I J}+B_{\mu}^{I K} B_{\nu}^{K J}-B_{\nu}^{I K} B_{\mu}^{K J}, \\
G_{\mu \nu} & =\partial_{\mu} B_{\nu}-\partial_{\nu} B_{\mu},
\end{aligned}
$$

and the covariant derivative $\mathcal{D}_{\mu}$ includes the $R$-symmetry transformation of the fields, e.g.,

$$
\mathcal{D}_{\mu} \epsilon^{I}=\left(\partial_{\mu}+\frac{1}{4} \hat{\omega}_{\mu a b} \gamma^{a b}\right) \epsilon^{I}+B_{\mu}^{I J} \epsilon^{J} .
$$

The quantity $\hat{\omega}_{\mu a b}(e, \psi)$ is the torsionful spin connection $\hat{\omega}_{\mu a b}(e, \psi)=\omega_{\mu a b}(e)+\frac{1}{8}\left(\bar{\psi}_{a}^{I} \gamma_{\mu} \psi_{b}^{I}+\bar{\psi}_{\mu}^{I} \gamma_{a} \psi_{b}^{I}-\bar{\psi}_{\mu}^{I} \gamma_{b} \psi_{a}^{I}\right)$,

with $\omega_{\mu a b}(e)$ denoting the torsion-free connection. In particular,

$$
\mathcal{D}_{\mu} e_{\nu}{ }^{a}-\mathcal{D}_{\nu} e_{\mu}{ }^{a}=\frac{1}{4} \bar{\psi}_{\mu}^{I} \gamma^{a} \psi_{\nu}^{I}
$$

Moreover, the Riemann curvature of $\hat{\omega}_{\mu a b}(e, \psi)$

$$
\hat{R}_{\mu \nu}^{a}{ }_{b} \equiv 2\left(\partial_{[\mu} \hat{\omega}_{\nu]_{b}}^{a}+\hat{\omega}_{[\mu}{ }^{a}{ }_{c} \hat{\omega}_{\nu]}^{c}{ }_{b}\right)
$$

satisfies the Bianchi identities

$$
\begin{aligned}
\hat{R}_{\mu \nu \rho \sigma}+\hat{R}_{\rho \mu \nu \sigma}+\hat{R}_{\nu \rho \mu \sigma} & =\frac{3}{2}\left(\Upsilon_{\mu \nu \rho \sigma}+\Upsilon_{\rho \mu \nu \sigma}+\Upsilon_{\nu \rho \mu \sigma}\right), \\
\hat{R}_{\mu \nu]} & =-\frac{3}{4} \Upsilon^{\rho} \rho_{\rho \mu \nu},
\end{aligned}
$$

where

$$
\Upsilon_{\nu \rho \sigma}^{\mu} \equiv \bar{\psi}_{[\nu}^{I} \gamma^{\mu} \psi_{\rho \sigma]}^{I} .
$$

The key reason for introducing the torsionful spin connection is that it transforms nicely under both Q and S supersymmetry, namely

$\begin{aligned} \delta_{Q}(\epsilon) \hat{\omega}_{\mu a b} & =-\frac{1}{4} \bar{\epsilon}^{I}\left(\gamma_{\mu} \psi_{a b}^{I}+\gamma_{a} \psi_{\mu b}^{I}-\gamma_{b} \psi_{\mu a}^{I}\right), \\ \delta_{S}(\eta) \hat{\omega}_{\mu a b} & =-\frac{1}{4} \bar{\eta}^{I}\left(\gamma_{a b} \psi_{\mu}^{I}+e_{\mu a} \psi_{b}^{I}-e_{\mu b} \psi_{a}^{I}\right) .\end{aligned}$

The local transformations of the $\mathcal{N}=6$ Weyl multiplet close off shell. In particular, the commutators between the fermionic transformations satisfy [78]

$$
\begin{aligned}
{\left[\delta_{Q}\left(\epsilon_{1}\right), \delta_{Q}\left(\epsilon_{2}\right)\right] } & =\delta_{D}(\xi)+\delta_{L}(\lambda)+\delta_{R}(\theta)+\delta_{Q}\left(\epsilon^{\prime}\right)+\delta_{S}\left(\eta^{\prime}\right), \\
{\left[\delta_{Q}(\epsilon), \delta_{S}(\eta)\right] } & =\delta_{W}(\sigma)+\delta_{L}\left(\lambda^{\prime}\right)+\delta_{R}\left(\theta^{\prime}\right)+\delta_{S}\left(\eta^{\prime \prime}\right) \\
{\left[\delta_{S}\left(\eta_{1}\right), \delta_{S}\left(\eta_{2}\right)\right] } & =0
\end{aligned}
$$

where the composite transformation parameters on the right-hand side are given by 


$$
\begin{aligned}
\xi^{\mu} & =\frac{1}{4} \bar{\epsilon}_{2}^{I} \gamma^{\mu} \epsilon_{1}^{I}, \quad \lambda_{a b}=-\xi^{\mu} \hat{\omega}_{\mu a b}, \\
\theta^{I J} & =-\xi^{\mu} B_{\mu}^{I J}+\frac{1}{4 \sqrt{2}} \varepsilon^{I J K L M N} \bar{\epsilon}_{2}^{K} \epsilon_{1}^{L} E^{M N}, \\
\theta & =-\xi^{\mu} B_{\mu}-\frac{1}{2 \sqrt{2}} \bar{\epsilon}_{2}^{K} \epsilon_{1}^{L} E^{K L}, \quad \epsilon^{\prime I}=-\xi^{\mu} \psi_{\mu}^{I}, \\
\eta^{\prime I} & =\frac{1}{2} \xi^{\mu} \gamma^{\rho \sigma} \gamma_{\mu} \psi_{\rho \sigma}^{I}-\frac{1}{16} \bar{\epsilon}_{2}^{[I} \epsilon_{1}^{J]} \gamma^{\rho \sigma} \psi_{\rho \sigma}^{J}-\frac{1}{2 \sqrt{2}} \bar{\epsilon}_{2}^{K} \epsilon_{1}^{L} \lambda^{I K L}-\frac{1}{16} \bar{\epsilon}_{2}^{(I} \gamma^{\mu} \epsilon_{1}^{J)}\left(2 \gamma^{\rho \sigma} \gamma_{\mu}+\gamma_{\mu} \gamma^{\rho \sigma}\right) \psi_{\rho \sigma}, \\
\sigma & =-\frac{1}{4} \bar{\epsilon}^{I} \eta^{I}, \quad \lambda_{a b}^{\prime}=\frac{1}{4} \bar{\epsilon}^{I} \gamma_{a b} \eta^{I}, \quad \theta^{I J}=-\frac{1}{2} \bar{\epsilon}^{[I} \eta^{J]}, \\
\theta^{\prime} & =0, \quad \eta^{\prime I I}=\frac{1}{8} \gamma^{\mu} \epsilon^{I}\left(\bar{\eta}^{J} \psi_{\mu}^{J}\right) .
\end{aligned}
$$

A supersymmetric Chern-Simons action for the $\mathcal{N}=6$ Weyl multiplet was found in [82,83]. In the component formulation of [82] it takes the form

$$
\begin{aligned}
\mathcal{L}_{\mathrm{CSG}}= & \frac{k}{4 \pi}\left[\frac{1}{2} \varepsilon^{\mu \nu \rho}\left(\hat{\omega}_{\mu}{ }_{b}{ }_{b} \partial_{\nu} \hat{\omega}_{\rho}{ }_{a}{ }_{a}+\frac{2}{3} \hat{\omega}_{\mu}{ }_{b}{ }_{b} \hat{\omega}_{\nu}{ }_{c}{ }_{c} \hat{\omega}_{\rho}{ }^{c}{ }_{a}\right)+\frac{1}{4} e \bar{\psi}_{\mu \nu} \gamma^{\rho \sigma} \gamma^{\mu \nu} \psi_{\rho \sigma}\right. \\
& -\varepsilon^{\mu \nu \rho}\left(B_{\mu}^{I J} \partial_{\nu} B_{\rho}^{J I}+\frac{2}{3} B_{\mu}^{I J} B_{\nu}^{J K} B_{\rho}^{K I}\right)-2 \varepsilon^{\mu \nu \rho} B_{\mu} \partial_{\nu} B_{\rho} \\
& +\frac{1}{3} e \bar{\lambda}^{I J K} \lambda^{I J K}-2 e \bar{\lambda}^{I} \lambda^{I}-8 e D^{I J} E^{I J}+\frac{1}{3 \sqrt{2}} e \varepsilon^{I J K L M N} E^{I J} E^{K L} E^{M N} \\
& +\frac{1}{6} e \varepsilon^{I J K L M N} \bar{\lambda}^{I J K} \gamma^{\mu} \psi_{\mu}^{L} E^{M N}+2 e \bar{\lambda}^{I} \gamma^{\mu} \psi_{\mu}^{J} E^{I J} \\
& \left.+e \bar{\psi}_{\mu}^{I} \gamma^{\mu \nu} \psi_{\nu}^{J}\left(E^{I K} E^{J K}-\frac{1}{4} \delta^{I J} E^{K L} E^{K L}\right)\right],
\end{aligned}
$$

where $e \equiv \operatorname{det}\left(e_{\mu}{ }^{a}\right)$ and we have chosen the convention $\kappa=1$ in (5.13). All off-shell Chern-Simons actions for Weyl multiplets with less supersymmetry found earlier [75,76,79-81] can be obtained by consistently truncating the $\mathcal{N}=6$ multiplet as indicated in Table II.

The Chern-Simons Lagrangian (5.50) is invariant under diffeomorphisms tangent to the boundary, but all other symmetries, namely Weyl, Lorentz, $R$ symmetry, and Q and S supersymmetry, result in a nonvanishing total derivative term. In particular, we find

$$
\begin{aligned}
\delta_{W}(\sigma) \mathcal{L}_{\mathrm{CSG}}= & \frac{k}{4 \pi} \varepsilon^{\mu \nu \rho} \partial_{\mu}\left(\frac{1}{2} \delta_{W}(\sigma) \hat{\omega}_{\nu}{ }^{a}{ }_{b} \hat{\omega}_{\rho}{ }^{b}{ }_{a}\right)=\frac{k}{4 \pi} \varepsilon^{\mu \nu \rho} \partial_{\mu}\left(\hat{\omega}_{\nu \rho}{ }^{\sigma} \partial_{\sigma} \sigma\right), \\
\delta_{L}(\lambda) \mathcal{L}_{\mathrm{CSG}}= & \frac{k}{8 \pi} \varepsilon^{\mu \nu \rho} \partial_{\mu}\left(\lambda^{a}{ }_{b} \partial_{\nu} \hat{\omega}_{\rho}{ }^{b}{ }_{a}\right), \\
\delta_{R}(\theta) \mathcal{L}_{\mathrm{CSG}}= & \frac{k}{4 \pi} \varepsilon^{\mu \nu \rho} \partial_{\mu}\left(\theta^{I J} \partial_{\nu} B_{\rho}^{I J}-2 \theta \partial_{\nu} B_{\rho}\right), \\
\delta_{Q}(\epsilon) \mathcal{L}_{\mathrm{CSG}}= & \frac{k}{4 \pi} \varepsilon^{\mu \nu \rho} \partial_{\mu}\left(\frac{1}{2} \delta_{Q}(\epsilon) \hat{\omega}_{\nu}{ }^{a}{ }_{b} \hat{\omega}_{\rho}{ }^{b}{ }_{a}+\delta_{Q}(\epsilon) B_{\nu}^{I J} B_{\rho}^{I J}-2 \delta_{Q}(\epsilon) B_{\nu} B_{\rho}\right) \\
& +\frac{k}{4 \pi} \partial_{\mu}\left(-\frac{1}{6} e \varepsilon^{I J K L M N} \bar{\epsilon}^{N} \gamma^{\mu} \lambda^{K L M} E^{I J}+2 e \bar{\epsilon}^{I} \gamma^{\mu} \lambda^{J} E^{I J}\right. \\
& \left.+2 e \bar{\epsilon}^{I} \gamma^{\mu \nu} \psi_{\nu}^{J}\left(E^{I K} E^{J K}-\frac{1}{4} \delta^{I J} E^{K L} E^{K L}\right)\right), \\
\delta_{S}(\eta) \mathcal{L}_{\mathrm{CSG}}= & \frac{k}{4 \pi} \varepsilon^{\mu \nu \rho} \partial_{\mu}\left(\frac{1}{2} \delta_{S}(\eta) \hat{\omega}_{\nu}{ }^{a}{ }_{b} \hat{\omega}_{\rho}{ }^{b}{ }_{a}+\delta_{S}(\eta) B_{\nu}^{I J} B_{\rho}^{I J}+\bar{\eta}^{I} \psi_{\nu \rho}^{I}\right) .
\end{aligned}
$$


Upon dimensional reduction to two dimensions these boundary terms produce the Lorentz anomaly of the $2 \mathrm{D}$ $\mathcal{N}=(6,6)$ multiplet and all associated mixed anomalies, demonstrating that they are obtainable through an inflow mechanism. The corresponding anomalies for any $\mathcal{N}=(p, p)$ multiplet with $p \leq 6$ can be obtained by a suitable truncation of the $3 \mathrm{D} \mathcal{N}=6$ multiplet as indicated in Table II. For chiral multiplets with $\mathcal{N}=(p, q), q<p$, one must first dimensionally reduce the boundary terms obtained from the $\mathcal{N}=p$ Chern-Simons action to two dimensions to obtain the anomalies for the $\mathcal{N}=(p, p)$ multiplet, and then further truncate the $2 \mathrm{D}$ multiplet to $\mathcal{N}=(p, q)$.

We should stress that the Weyl anomaly in (5.51) is not the usual 2D Weyl anomaly, but rather a supersymmetric version of the mixed Lorentz-Weyl anomaly [91]. Moreover, once truncated to $\mathcal{N}=1$ supersymmetry, (5.51) reproduces the result of [20] for minimal Poincaré supergravity. To see this, one must first truncate the $\mathcal{N}=6$ conformal supergravity multiplet to the $\mathcal{N}=1$ one as indicated in Table II, and then construct the corresponding $\mathcal{N}=1$ Poincaré supergravity by coupling a compensating chiral multiplet. This is directly analogous to the construction of old minimal supergravity in four dimensions from $\mathcal{N}=1$ conformal supergravity (see [31] for a recent review of this construction). The supersymmetry transformation of the Poincaré multiplet is a field-dependent linear combination of the Qand S-supersymmetry transformations of conformal supergravity, namely $\delta_{Q}^{\text {Poincaré }(\epsilon)=\delta_{Q}(\epsilon)+\delta_{S}(\eta=S \epsilon)}$, where $S$ is an auxiliary real scalar field, a component of the compensating chiral multiplet. As a result, the supersymmetric completion of the Lorentz anomaly for minimal Poincaré supergravity is the sum of the Q and S anomalies in (5.51), with all R-symmetry gauge fields and (conformal supergravity) auxiliary fields set to zero.

\section{ANOMALY DESCENT WITH MISMATCHING GHOST}

We have seen earlier that, for diffeomorphisms, the standard anomaly descent mechanism does not quite reflect the relevant WZ consistency condition: the latter should hold for full diffeomorphisms, while the usual descent procedure relies only on the $G L(d)$ rotational part of diffeomorphisms. Even though the resulting $G L(d)$ anomaly descent yields the correct diffeomorphism anomaly [6], it is not entirely transparent how this can be packaged into the BRST algebra. For the case of the supersymmetry Ward identity and anomalous terms thereof, we find another deviation from the standard anomaly descent, simply because there seems to be no place for the supersymmetry parameters in the usual BRST algebra for anomaly descent.

The two share a common need for generalizing the anomaly descent procedure. We should comment here that such a generalization of the BRST algebra and the descent procedure thereof has been studied in the context of supersymmetrized anomaly in the past. The most notable work is Refs. $[14,15]$ which inspired the bulk of what we do here. Another such attempt was given later in Refs. [17,18], although their choice of the BRST operator and of the ghost differ from ours. We should clarify that we focus on rigid supersymmetry and gauge/flavor anomalies here. See Refs. [92,93], e.g., for recent related discussions in the supergravity context.

Recall that the usual anomaly descent arises from a BRST algebra where we replace

$$
\mathrm{d} \rightarrow \mathrm{d}+\mathbf{s}, \quad \mathcal{A} \rightarrow \hat{\mathcal{A}} \equiv \mathcal{A}+v,
$$

with $\mathbf{s}^{2}=0=(\mathrm{d}+\mathbf{s})^{2}$, so that

$$
\mathcal{F}=\hat{\mathcal{F}} \equiv(\mathrm{d}+\mathbf{s}) \hat{\mathcal{A}}+\hat{\mathcal{A}}^{2} .
$$

Together they lead to

$$
\begin{aligned}
P_{d+2}(\mathcal{F}) & =P_{d+2}(\hat{\mathcal{F}})=(\mathrm{d}+\mathbf{s}) \mathbf{w}_{d+1}(\hat{\mathcal{A}}, \hat{\mathcal{F}}) \\
& =(\mathrm{d}+\mathbf{s}) \mathbf{w}_{d+1}(\hat{\mathcal{A}}, \mathcal{F})
\end{aligned}
$$

for any given anomaly polynomial $P_{d+2}$. As discussed in Sec. II, the rightmost expression is now expanded in the ghost number and equated to the leftmost expression with no ghost dependence, resulting in the standard descent formulas.

The generalized descent structure we are interested in arises when, in addition to $\mathbf{s}$, there exists an additional BRST odd operator $\mathbf{c}$ such that

$$
(\mathrm{d}+\mathbf{s}+\mathbf{c})^{2}=0,
$$

as well. Of course, $\mathbf{s}$ was meant to represent multiple types of gauge transformation so the point of this additional operator $\mathbf{c}$ is that the action of $\mathbf{c}$ on the connections and the ghosts is not standard, i.e., as in $\mathbf{s} v=-v^{2}$. In our actual examples below, c corresponds either to diffeomorphisms or to rigid supersymmetry. Note that we do not necessarily demand that $\mathbf{c}^{2}=0$ or $(\mathbf{s}+\mathbf{c})^{2}=0$ holds either, although they do hold when $\mathbf{c}$ represents diffeomorphisms.

In order to generalize the BRST algebra in the presence of $\mathbf{c}$, we also add a new ghost $u$ and extend the BRST gauge field further to $\hat{\mathcal{A}}+u$. However, the relation between the $u$ ghost and the operator $\mathbf{c}$ would be rather different from that between $v$ and $\mathbf{s}$. We need an additional ghost to define $\mathbf{c}$ but these do not necessarily appear as $u$. In fact, for our two classes of examples in this section, we will take $u=0$, while $\mathbf{c}$ and the ghost parameters thereof remain nontrivial. Defining the BRST field strength

$$
\hat{\mathcal{G}} \equiv(\mathrm{d}+\mathbf{s}+\mathbf{c})(\hat{\mathcal{A}}+u)+(\hat{\mathcal{A}}+u)^{2},
$$


where $\hat{\mathcal{A}}=\mathcal{A}+v$ as before, this field strength satisfies the generalized Bianchi identity

$$
(\mathrm{d}+\mathbf{s}+\mathbf{c}) \hat{\mathcal{G}}+(\hat{\mathcal{A}}+u) \hat{\mathcal{G}}-\hat{\mathcal{G}}(\hat{\mathcal{A}}+u)=0,
$$

by virtue of the nilpotency of $d+\mathbf{s}+\mathbf{c}$. We must emphasize that we no longer have $\hat{\mathcal{G}}$ equal to $\mathcal{F}$, in view of how the $u$ ghost, or the absence thereof in examples below, mismatches the BRST operator $\mathrm{d}+\mathbf{s}+\mathbf{c}$.

It follows that

$$
(\mathrm{d}+\mathbf{s}+\mathbf{c}) P_{d+2}(\hat{\mathcal{G}})=0
$$

and hence, locally

$$
P_{d+2}(\hat{\mathcal{G}})=(\mathrm{d}+\mathbf{s}+\mathbf{c}) \mathbf{w}_{d+1}(\hat{\mathcal{A}}+u, \hat{\mathcal{G}})
$$

Subtracting either of the two relations in (6.3) leads to the identity

$$
\begin{aligned}
& (\mathrm{d}+\mathbf{s}+\mathbf{c}) \mathbf{w}_{d+1}(\hat{\mathcal{A}}+u, \hat{\mathcal{G}})-\mathrm{d} \mathbf{w}_{d+1}(\mathcal{A}, \mathcal{F}) \\
& =P_{d+2}(\hat{\mathcal{G}})-P_{d+2}(\mathcal{F}) .
\end{aligned}
$$

The right-hand side does not vanish, since $\hat{\mathcal{G}} \neq \mathcal{F}$ in general. Instead, we find that

$$
\begin{aligned}
\hat{\mathcal{G}} & =(\mathrm{d}+\mathbf{s}+\mathbf{c})(\hat{\mathcal{A}}+u)+(\hat{\mathcal{A}}+u)^{2} \\
& =\hat{\mathcal{F}}+\mathrm{d} u+(\mathbf{s}+\mathbf{c})(\hat{\mathcal{A}}+u)+\hat{\mathcal{A}} u+u \hat{\mathcal{A}}+u^{2} \\
& =\mathcal{F}+\mathbf{c} \mathcal{A}+\mathbf{s} u+\{v, u\}+\mathbf{c} v+\mathbf{c} u+\mathrm{d}_{\hat{\mathcal{A}}} u+u^{2} .
\end{aligned}
$$

The difference $\hat{\mathcal{G}}-\mathcal{F}$ depends on the operator $\mathbf{c}$ and the choice of $u$. Conversely, $u$ may be fixed by requiring the difference $\hat{\mathcal{G}}-\mathcal{F}$ to be of a specific form. ${ }^{9}$

Given that $\hat{\mathcal{G}}-\mathcal{F}$ is nonzero in general, we proceed by defining

$$
P_{d+2}(\hat{\mathcal{G}})-P_{d+2}(\mathcal{F}) \equiv \sum_{k \geq 1} X_{d+2-k}^{(k)},
$$

where the integer $k$ again indicates the (generalized) ghost number. The nonzero $X$ 's can be viewed as obstructions to the standard anomaly descent procedure.

As we shall see, in relevant examples, these $X$ 's can be themselves reconstructed by the action of $(\mathbf{s}+\mathbf{c})$ and $\mathrm{d}$ on more elemental quantities, to be denoted as $Y$ 's and $Z$ 's respectively, provided that we remember that we want a local functional on $d$-dimensional spacetime. This way, we once again obtain a generalized anomaly annihilated by

\footnotetext{
${ }^{9}$ As mentioned earlier, we shall take $u=0$ below, as it allows a single modification of the descent procedure to cover both diffeomorphisms and supersymmetry. Another natural choice for $u$ is discussed in the Appendix.
}

$(\mathbf{s}+\mathbf{c})$. The resulting anomaly would receive contributions from both the left- and right-hand sides of (6.9).

A simplest example of this, it turns out, is the familiar diffeomorphism anomaly. Although we are accustomed to computing the diffeomorphism anomaly via $G L(d)$ descent, we have reviewed in Sec. III how the result actually obeys the consistency condition of full diffeomorphisms. Next, we will illustrate the above generalized anomaly descent for this example and move on to supersymmetry later. In fact, our generalized descent procedure is inspired by an early attempt of constructing a supersymmetric descent procedure [14-16].

\section{A. Diffeomorphism anomaly revisited}

Let us start by considering how the diffeomorphism WZ consistency condition can be elevated to a BRST form. For this we elevate $\mathcal{L}_{\xi}$ to an operator $\mathbf{c}$, with the unit ghost number, such that

$$
0=\mathrm{d}^{2}=\left(\mathrm{d}+\mathbf{s}_{g}\right)^{2}=\left(\mathrm{d}+\mathbf{s}_{g}+\mathbf{c}\right)^{2},
$$

with $\mathbf{s}_{g}$, for now, restricted to the internal gauge transformation. The entire diffeomorphisms are carried by c.

Given how $\mathcal{L}_{\xi}=\mathcal{L}_{\xi}^{\prime}+\delta_{-\partial \xi}^{G L(d)}$ in general, more care is needed to define the action of $\mathbf{c}$ on the Christoffel connections and the accompanying ghost. On the connections

$$
\mathbf{c} A=\mathcal{L}_{\mathbf{x}} A=\mathcal{L}_{\mathbf{x}}^{\prime} A, \quad \mathbf{c} \Gamma=\mathcal{L}_{\mathbf{x}}^{\prime} \Gamma+\mathrm{d}_{\Gamma}(-\partial \mathbf{x})
$$

while the action of $\mathbf{c}$ on the ghosts is

$$
\mathbf{c} v_{g}=\mathcal{L}_{\mathbf{x}} v_{g}, \quad \mathbf{c} \mathbf{x}^{\mu}=\mathbf{x}^{\alpha} \partial_{\alpha} \mathbf{x}^{\mu}, \quad \mathbf{s}_{g} \mathbf{x}=0
$$

where $v_{g}$ is the gauge part of the ghost. It follows that

$$
\mathbf{c}(-\partial \mathbf{x})=-(-\partial \mathbf{x})^{2}+\mathcal{L}_{\mathbf{x}}^{\prime}(-\partial \mathbf{x})
$$

where again $\mathcal{L}^{\prime}$ treats $(-\partial \mathbf{x})$ as if the latter is a matrixvalued function. One can see that $\mathbf{c}$ in part plays the role of $\mathbf{s}$ on the diffeomorphism sector but incorporates a full diffeomorphism rather than $G L(d)$ only.

With this, $\mathbf{c}^{2}=0$ by itself. For instance,

$$
\begin{aligned}
\mathbf{c}^{2} v & =\mathbf{c}\left(\mathbf{x}^{\alpha} \partial_{\alpha} v\right)=\left(\mathbf{x}^{\alpha} \partial_{\alpha} \mathbf{x}^{\mu}\right) \partial_{\mu} v-\left(\mathbf{x}^{\alpha} \partial_{\alpha}\left(\mathbf{x}^{\mu} \partial_{\mu} v\right)\right) \\
& =-\mathbf{x}^{\alpha} \mathbf{x}^{\mu} \partial_{\alpha} \partial_{\mu} v=0
\end{aligned}
$$

due to the Grassmannian properties of $\mathbf{x}^{\mu}$. On tensors (as well as on the Christoffel connection), it suffices to consider $\mathbf{x}=\chi \zeta+\chi^{\prime} \xi$ with a pair of Grassmannian coefficients $\chi$ and $\chi^{\prime}$, and a pair of arbitrary vectors $\zeta$ and $\xi$, whereby $\mathbf{c}\left(\mathbf{x}^{\mu}\right)=\chi \chi^{\prime}[\zeta, \xi]^{\mu}$ holds so that 


$$
\begin{aligned}
\mathbf{c c} V & =\mathcal{L}_{\mathbf{c}(\mathbf{x})} V-\mathcal{L}_{\chi \zeta+\chi^{\prime} \xi}\left(\mathcal{L}_{\chi \zeta+\chi^{\prime} \xi} V\right) \\
& =\chi \chi^{\prime}\left(\mathcal{L}_{[\zeta, \xi]} V-\left[\mathcal{L}_{\zeta}, \mathcal{L}_{\xi}\right] V\right)=0 .
\end{aligned}
$$

With $\mathbf{s}_{g}$ being restricted solely to gauge transformations and c representing diffeomorphisms, $\mathbf{s}_{g}$ and $\mathbf{c}$ anticommute. Together with $\mathbf{c}^{2}=0$, this suffices to verify the nilpotency of the BRST operator (6.12) d $+\mathbf{s}_{g}+\mathbf{c}$.

On the other hand, different from $v_{g}$, the $\mathbf{x}$ ghost cannot be naturally added to $\mathcal{A}$, since it is really a vector, hence a directional derivative. Instead we can define $v=v_{g}+(-\partial \mathbf{x})$ which includes all gauge rotations plus the $G L(d)$ rotation generated by diffeomorphisms. Concurrently it is natural to split

$$
\mathbf{c}=\mathbf{s}_{G L(d)}+\mathbf{c}^{\prime}
$$

so that $\mathbf{c}^{\prime}$ retains the purely translational part, $\mathcal{L}_{\mathbf{x}}^{\prime}$. Its rotational part can be treated on an equal footing with $\mathbf{s}_{g}$ leading to the redefinition of the BRST operator

$$
\mathrm{d}+\mathbf{s}_{g}+\mathbf{c}=\mathrm{d}+\mathbf{s}+\mathbf{c}^{\prime}
$$

with $\mathbf{s}=\mathbf{s}_{g}+\mathbf{s}_{G L(d)}$. One may now take $v=v_{g}+(-\partial \mathbf{x})$ and since there is no other ghost that can be added naturally, $u=0$. With such a mismatching ghost, we construct the BRST field strength

$\hat{\mathcal{G}} \equiv\left(\mathrm{d}+\mathbf{s}+\mathbf{c}^{\prime}\right) \hat{\mathcal{A}}+\hat{\mathcal{A}}^{2}=\hat{\mathcal{F}}+\mathbf{c}^{\prime} \hat{\mathcal{A}}=\mathcal{F}+\mathbf{c}^{\prime}(\mathcal{A}+v)$

which nevertheless obeys the same Bianchi identity as above

$$
\begin{aligned}
\left(\mathrm{d}+\mathbf{s}+\mathbf{c}^{\prime}\right) \hat{\mathcal{G}}+\hat{\mathcal{A}} \hat{\mathcal{G}}-\hat{\mathcal{G}} \hat{\mathcal{A}}=\mathbf{c}^{\prime}[\mathcal{F}] \\
-\mathbf{c}^{\prime}\left[(\mathrm{d}+\mathbf{s})(\hat{\mathcal{A}})+(\hat{\mathcal{A}})^{2}\right]=0 .
\end{aligned}
$$

We have invoked here $\left(\mathbf{c}^{\prime}\right)^{2}+\left\{d+\mathbf{s}, \mathbf{c}^{\prime}\right\}=0$ that follows from (6.12).

An immediate consequence of the Bianchi identity is that an invariant polynomial $P_{d+2}(\hat{\mathcal{G}})$ satisfies

$$
\begin{aligned}
\left(\mathrm{d}+\mathbf{s}+\mathbf{c}^{\prime}\right) P_{d+2}(\hat{\mathcal{G}}) & =0, \\
P_{d+2}(\hat{\mathcal{G}}) & =\left(d+\mathbf{s}+\mathbf{c}^{\prime}\right) \mathbf{w}_{d+1}(\hat{\mathcal{A}}, \hat{\mathcal{G}}) .
\end{aligned}
$$

The two sides of the second equation can be expanded respectively as

$$
\begin{gathered}
P_{d+2}(\hat{\mathcal{G}})=P_{d+2}(\mathcal{F})+\sum_{k \geq 1} X_{d+2-k}^{(k)}(\mathbf{x}, v, \mathcal{A}, \mathcal{F}) \\
\mathbf{w}_{d+1}(\hat{\mathcal{A}}, \hat{\mathcal{G}})=\mathbf{w}_{d+1}(\mathcal{A}, \mathcal{F})+\sum_{k \geq 1} W_{d+1-k}^{(k)}(\mathbf{x}, v, \mathcal{A}, \mathcal{F})
\end{gathered}
$$

where $k$ keeps track of the net number of ghosts. A pair of descent towers now follows:

$$
\begin{array}{r}
\left(\mathbf{s}+\mathbf{c}^{\prime}\right) X_{d+2-k}^{(k)}+\mathrm{d} X_{d+1-k}^{(k+1)}=0, \\
X_{d+1-k}^{(k+1)}=\left(\mathbf{s}+\mathbf{c}^{\prime}\right) W_{d+1-k}^{(k)}+\mathrm{d} W_{d-k}^{(k+1)}
\end{array}
$$

with $X_{d+2}^{(0)} \equiv P_{d+2}(\mathcal{F})$ and $W_{d+1}^{(0)} \equiv \mathbf{w}_{d+1}(\mathcal{A}, \mathcal{F})$.

Given $\left(\mathbf{s}_{g}+\mathbf{c}\right)^{2}=0$, the first equation in (6.24) implies that

$\left(\mathbf{s}+\mathbf{c}^{\prime}\right) \int X_{d+2-k}^{(k)}=0 \rightarrow \int X_{d+2-k}^{(k)}=\left(\mathbf{s}+\mathbf{c}^{\prime}\right) \int Y_{d+2-k}^{(k-1)}$

for some $Y$ 's that are not d exact. Taking $k=1$ in (6.24) we obtain

$$
0=\left(\mathbf{s}+\mathbf{c}^{\prime}\right) \int_{M_{d}}\left(W_{d}^{(1)}-Y_{d}^{(1)}\right)
$$

a solution to the WZ consistency conditions, which now due to $\mathbf{s}+\mathbf{c}^{\prime}=\mathbf{s}_{g}+\mathbf{c}$ are extended to include general diffeomorphisms $\delta_{\xi}$.

This may appear to be a tautology, since $Y^{(1)}=W^{(1)}$ is seemingly also acceptable, leading to a trivial solution to the BRST version of the WZ consistency conditions. However, the point is that a different nontrivial solution for $Y^{(1)}$ can be found from

$$
\begin{aligned}
\sum_{k \geq 0} X_{d+1-k}^{(k+1)}= & P_{d+2}(\hat{\mathcal{G}})-P_{d+2}(\mathcal{F}) \\
= & \left(\mathrm{d}+\mathbf{s}+\mathbf{c}^{\prime}\right) \sum_{l \geq 0}\left(W_{d+1-l}^{(l)}-\mathbf{w}_{d+1-l}^{(l)}\right) \\
& +\mathbf{c}^{\prime} \sum_{l \geq 0} \mathbf{w}_{d+1-l}^{(l)} .
\end{aligned}
$$

In particular, the action of $\mathbf{c}^{\prime}$ on the $d$-form $\mathbf{w}_{d}^{(1)}$ is nothing but $\mathcal{L}_{\mathbf{x}}^{\prime}$, given that all $G L(d)$ indices are summed over. Using the identity $\mathcal{L}_{\mathbf{x}}^{\prime}(\cdots)=\mathbf{x}_{\lrcorner} \mathrm{d}(\cdots)-\mathrm{d}\left(\mathbf{x}_{\lrcorner} \cdots\right)$ we find

$$
\begin{aligned}
& X_{d}^{(2)}=\left(\mathbf{s}+\mathbf{c}^{\prime}\right)\left(W_{d}^{(1)}-\mathbf{w}_{d}^{(1)}\right)+\mathrm{d}\left(W_{d-1}^{(2)}-\mathbf{w}_{d-1}^{(2)}-\mathbf{x} \mathbf{w}_{d}^{(1)}\right) \\
& +\mathbf{x}\lrcorner \mathrm{d} \mathbf{w}_{d}^{(1)}
\end{aligned}
$$

which gives a different, nontrivial solution for $Y_{d}^{(1)}$.

We may recall once again that $\mathrm{d} \mathbf{w}_{d}^{(1)}$ actually vanishes identically once we restrict to the physical $d$ dimension and coordinates thereof, and the equation (6.28) yields

$0=\left(\mathbf{s}+\mathbf{c}^{\prime}\right) \int_{M_{d}}\left(W_{d}^{(1)}-Y_{d}^{(1)}\right)=\left(\mathbf{s}+\mathbf{c}^{\prime}\right) \int_{M_{d}} \mathbf{w}_{d}^{(1)}(v ; \mathcal{A}, \mathcal{F})$

or more concretely 
$0=\left(\mathbf{s}_{g}+\mathbf{c}\right)\left(\int \mathbf{w}_{d}^{(1)}(-\partial \mathbf{x} ; \Gamma, R ; F)+\int \mathbf{w}_{d}^{(1)}\left(v_{g} ; A, F ; R\right)\right)$,

bringing us to the known solution, but via a simpler BRST route with diffeomorphisms now honestly incorporated.

\section{B. Anomaly descent with supersymmetry}

Now we can apply the same procedure to include supersymmetry in the presence of gauge/flavor anomalies. For this, we use the same $\mathbf{s}=\mathbf{s}_{g}, v=v_{g}$ and add

$$
\mathbf{c}=\mathbf{q}+\mathbf{a}
$$

where a acts universally as a translation by a constant vector-valued ghost $a^{\mu}$,

$$
\mathbf{a}(\cdots)=a^{\mu} \partial_{\mu}(\cdots)=\mathcal{L}_{a}(\cdots)
$$

while $\mathbf{q}$ is the BRST version of the rigid supersymmetry. Let us now use these in the generalized anomaly descent.

Unlike with the diffeomorphism case above, however, the descent process remains universal only down to the first term in the supersymmetry completion of the anomaly, which as we have seen earlier can be expressed in terms of the BZ current. The rest, which is manifestly gauge invariant, depends very much on the spacetime dimension and amount of supersymmetry. We will therefore concentrate on outlining the general procedure only up to this universal supersymmetry completion. This way we can distill the previous attempts of constructing a supersymmetric descent formalism. The full form of the supersymmetry completion of the gauge/flavor anomaly in different cases can be found in Sec. V or in the literature. See, for example, Refs. [8-10,14-16].

Let us consider an arbitrary even dimension $d$ and the minimal supersymmetry thereof. For off-shell multiplets, the smallest possible field content arises for $d=6 \mathcal{N}=(1,0)$, while for one-shell multiplets, one can go up to $d=10$, $\mathcal{N}=1$. As such the fermions would obey a Majorana condition of some kind. The action of $\mathbf{q}$ involves the cnumber-valued Majorana spinor ghost $\alpha$ and takes the form

$$
\begin{aligned}
\mathbf{q} A_{\mu} & =-\frac{1}{2} \bar{\alpha} \gamma_{\mu} \lambda, \\
\mathbf{q} \lambda & =\frac{1}{4} \gamma^{\mu \nu} F_{\mu \nu}^{a}+\cdots \\
\mathbf{q} v & =\frac{1}{4} \bar{\alpha} \gamma^{\mu} \alpha A_{\mu}, \\
\mathbf{q} \alpha & =0, \\
& \cdots \\
\mathbf{q} a^{\mu} & =-\frac{1}{4} \bar{\alpha} \gamma^{\mu} \alpha
\end{aligned}
$$

where the ellipsis denotes transformations involving auxiliary fields for off-shell transformations, e.g., as in (5.29). Let us also note that the translation operator a acting on $a$ and on $\alpha$ vanishes as these ghosts are taken to be constant. ${ }^{10}$

Choosing $u=0$, the generalized descent for $\mathbf{c}$ works precisely the same way as already outlined. In particular, for rigid supersymmetry

$$
X_{d}^{(2)}=(\mathbf{s}+\mathbf{c})\left(W_{d}^{(1)}-\mathbf{w}_{d}^{(1)}\right)+\mathrm{d}\left(W_{d-1}^{(2)}-\mathbf{w}_{d-1}^{(2)}\right)+\mathbf{c} \mathbf{w}_{d}^{(1)}
$$

where in the last term $\mathbf{w}_{d}^{(1)}$ depends neither on $\mathbf{c}$ ghost nor on the superpartner. The $\mathbf{c}$ operation on it can be decomposed into three parts: one is the SUSY transformation $\mathbf{q}$ acting on $A$ and $F$, the other is also $\mathbf{q}$ acting on $v$, and finally the action of a as $a$ gradient on $v, A$, and $F$. In particular the last a acting on the differential $d$-form produces total derivative terms, which will eventually vanish upon $M_{d}$ integration.

The first part can be written, using the same antiderivative $l$, as

$$
\delta_{\mathbf{q} A}=-\mathrm{d} l_{\mathbf{q} A}+l_{\mathbf{q} A} \mathrm{~d}
$$

The sign flip [cf. Eq. (2.28)] is due to the fact that $l_{\mathbf{q} A}$ not only carries a single ghost number but also reduces the rank of the differential form and hence is BRST even. Modulo terms that drop out upon $M_{d}$ integration,

$$
\begin{aligned}
\mathbf{c w}_{d}^{(1)}= & -l_{\mathbf{q} A}\left(\left(\mathbf{s} \mathbf{w}_{d+1}^{(0)}(A, F)\right)+\mathbf{w}_{d}^{(1)}(\mathbf{q} v ; A, F)+\cdots\right. \\
= & (\mathbf{s}+\mathbf{c})\left(-l_{\mathbf{q} A} \mathbf{w}_{d+1}^{(0)}(A, F)\right) \\
& +\mathbf{c}\left(l_{\mathbf{q} A} \mathbf{w}_{d+1}^{(0)}(A, F)\right)+\mathbf{w}_{d}^{(1)}(\mathbf{q} v ; A, F)+\cdots
\end{aligned}
$$

since $\mathbf{w}_{d}^{(1)}$ is linear in $v$. Thus, we arrive at

$$
\begin{aligned}
X_{d}^{(2)}= & (\mathbf{s}+\mathbf{c})\left(W_{d}^{(1)}-\mathbf{w}_{d}^{(1)}-l_{\mathbf{q} A} \mathbf{w}_{d+1}^{(0)}\right) \\
& +\mathbf{q}\left(l_{\mathbf{q} A} \mathbf{w}_{d+1}^{(0)}(A, F)\right)+\mathbf{w}_{d}^{(1)}(\mathbf{q} v ; A, F)+\cdots
\end{aligned}
$$

where we again used the fact that a produces a total derivative term at most. Note that the last term in the first line is

$$
\int l_{\mathbf{q} A} \mathbf{w}_{d+1}^{(0)}=\mathbf{q} A \cdot X(A, F)
$$

\footnotetext{
${ }^{10}$ Elevating these to position-dependent quantities must entail couplings to external supergravity and is needed if we wish to extend this to the cases with a diffeomorphism anomaly. We believe that the same supersymmetric descent mechanism can be extended to that case as well.
} 
where $X$ is the BZ current. This is exactly the $\lambda$-linear term that we would have found by imposing the mixed SUSYgauge WZ consistency condition as in Refs. [8-10].

The question is then, does the second line produce a term which has the form $(\mathbf{s}+\mathbf{c})$ acting on something else? A useful middle step is to separate out those terms with no gaugino attached by rewriting

$$
(\mathbf{q} A) \cdot \mathbf{q} X(A, F)+\left((\mathbf{q q} A) \cdot X(A, F)+\mathbf{w}_{d}^{(1)}(\mathbf{q} v ; A, F)\right) .
$$

Now, we know from the above that

$$
\mathbf{q} v=\xi_{\lrcorner} A, \quad \xi^{\mu} \equiv \frac{1}{4} \bar{\alpha} \gamma^{\mu} \alpha
$$

while

$\mathbf{q q} A_{\rho}=\mathbf{q}\left(-\frac{1}{2} \bar{\alpha} \gamma_{\rho} \lambda\right)=\frac{1}{16} \bar{\alpha}\left[\gamma_{\rho}, \gamma^{\mu \nu}\right] \alpha F_{\mu \nu}=-\left(\xi_{\lrcorner} F\right)_{\rho}$

where we use $\bar{\alpha} \gamma_{\rho \mu \nu} \alpha=0$ for c-valued Majorana $\alpha .^{11}$ Combining the two, we find

$$
\begin{aligned}
& \left((\mathbf{q q} A) \cdot X(A, F)+\mathbf{w}_{d}^{(1)}(\mathbf{q} v ; A, F)\right) \\
& =-\left(\xi_{\lrcorner} F\right) \cdot X-\left(\xi_{\lrcorner} A\right) \cdot\langle\nabla \cdot J\rangle=0
\end{aligned}
$$

for exactly the same reason how the covariant gauge current, rather than the consistent current, had to enter in the diffeomorphism Ward identity as in Sec. II.

As such, we are left with a single term that can further contribute to $Y^{(1)}$. Its form from Sec. III suggests that the answer must be gauge invariant so we hope to find $\Delta Y_{\text {inv }}^{(1)}$ such that

$(\mathbf{q} A) \cdot \mathbf{q} X(A, F)=\int(\mathbf{s}+\mathbf{c}) \Delta Y_{\mathrm{inv}}^{(1)}=\int \mathbf{q} \Delta Y_{\mathrm{inv}}^{(1)}$

where again, a acting on $\Delta Y_{\text {inv }}^{(1)}$ turns into a total derivative. This, together with (6.42), would precisely elevate the solutions of [8-10] to the SUSY-SUSY WZ consistency condition to the BRST level in our generalized descent context.

For this to work, one necessary condition is that the lefthand side is itself invariant under gauge transformations. In fact, we claim that this pattern of $\mathbf{q} A \cdot \mathbf{q} X$ being gauge invariant is universal, even though the BZ current $X$ is not gauge covariant. One can see this formally via the very definition of the BZ current,

$$
X=J_{\mathrm{cov}}-J=J_{\mathrm{cov}}-\frac{\delta W}{\delta A}
$$

whereby

$$
\mathbf{q} A \cdot \mathbf{q} X=\mathbf{q} A \cdot \mathbf{q} J_{\mathrm{cov}}-(\mathbf{q} A)(\mathbf{q} A) \cdot \frac{\delta^{2} W}{\delta A \delta A} .
$$

The first piece is manifestly invariant with $\mathbf{q} A$ being covariant, while the second, potentially noninvariant piece vanishes since, componentwise, $\mathbf{q} A_{\mu}$ is BRST odd while, $A_{\mu}$ is BRST even.

Given the anomaly polynomial $P_{d+2}(F)$, a sum of products of symmetrized traces of its $d / 2+1$ arguments as usual, what would be the explicit expression for $\mathbf{q} A \cdot \mathbf{q} X$ ? We start from the usual transgression formula for $\mathbf{w}_{d+1}^{(0)}$ and arrive at

$$
\mathbf{q} A \cdot X=(d / 2)(d / 2+1) \int_{0}^{1} d t \int_{M_{d}} P_{d+2}\left(A, t \mathbf{q} A, F_{t}, F_{t}, \ldots, F_{t}\right)
$$

with $A_{t} \equiv t A$ and $F_{t} \equiv d A_{t}+A_{t}^{2}=t F+\left(t^{2}-t\right) A^{2}$. The integrand of $\mathbf{q} A \cdot \mathbf{q} X$ becomes

$$
\begin{aligned}
& P_{d+2}\left(\mathbf{q} A, t \mathbf{q} A, F_{t}, \ldots, F_{t}\right)+(d / 2-1) P_{d+2}\left(A, t \mathbf{q} A, \mathrm{~d}_{t A}(t \mathbf{q} A), F_{t}, \ldots, F_{t}\right) \\
& =\frac{1}{2} \partial_{t} P_{d+2}\left(t \mathbf{q} A, t \mathbf{q} A, F_{t}, F_{t}, \ldots, F_{t}\right)+\cdots
\end{aligned}
$$

\footnotetext{
${ }^{11}$ The transformation of $\lambda$ into the auxiliary field can potentially spoil this; however, one can see that the Majorana nature of $\alpha$ is such that this piece vanishes identically, for the same reason that $\bar{\alpha} \gamma_{\rho \mu \nu} \alpha=0$.
} 
which gives, dropping the d-exact term in the ellipsis,

$$
\mathbf{q} A \cdot \mathbf{q} X=\frac{(d / 2)(d / 2+1)}{2} \int_{M_{d}} P_{d+2}(\mathbf{q} A, \mathbf{q} A, F, F, \ldots, F)
$$

with $d / 2-1 F$ 's inside.

In short, we have shown that the supersymmetry completion of the anomaly has the universal decomposition

$$
\int\left(\mathbf{w}_{d}^{(1)}+l_{\mathbf{q} A} \mathbf{w}_{d+1}^{(0)}-\Delta Y_{\text {inv }}^{(1)}\right)
$$

that is, the standard anomaly, the BZ current contracted against $\mathbf{q} A$, and the last, invariant part of the supersymmetry completion that solves

$$
\mathbf{q} \int \Delta Y_{\mathrm{inv}}^{(1)}=\frac{(d / 2)(d / 2+1)}{2} \int_{M_{d}} P_{d+2}(\mathbf{q} A, \mathbf{q} A, F, F, \ldots, F)
$$

where $P_{d+2}$ is the anomaly polynomial responsible for $\mathbf{w}_{d+1-k}^{(k)}$ 's via the standard anomaly descent.

As we warned earlier, the precise solution for $\Delta Y_{\text {inv }}^{(1)}$ strongly depends on spacetime dimensions, number of supersymmetries, and on shell vs off shell. For $d \leq 4$, we have already given several answers via Chern-Simons anomaly inflow. For examples in higher dimensions, such as $d=10$ and $\mathcal{N}=1$ or $d=6$ and $\mathcal{N}=(1,0)$, we refer the readers to Refs. [14-16], which in fact inspired the general descent procedure we gave in the section.

Even though we started with minimal supersymmetry in even $d$, the actual spacetime dimension is flexible. In other words, the same descent mechanism works as long as the theory comes from dimensional reduction and truncation of some higher $d^{\prime}>d$ theory with minimal supersymmetry; we only need to make sure that the $\gamma$ matrices above are those of the original $d^{\prime}$-dimensional Majorana spinor. For example, one can make use of the $d=6 \mathcal{N}=(1,0)$ example for $d=4 \mathcal{N}=2$, although in this latter case, the chirality is lost and gauge/flavor anomaly would be absent. A more interesting example would come about by a further truncation of supersymmetry as we reduce $d^{\prime} \rightarrow d$. In fact the same sort of idea was employed in the previous section where, for example, we start with anomaly inflow from a single $d=3 \mathcal{N}=3$ Chern-Simons to generate the anomaly of $d=2 \mathcal{N}=(p, q)$ for various $p, q \leq 3$.

Finally, we should mention again that the supersymmetry completion of anomalies has been the subject of past studies, and much of what we outlined in this section has already appeared in bits and pieces. For instance, an equivalent form of (6.48) has appeared as early as in Refs. [8,10], albeit as an on-shell statement and without a BRST formulation. The present discussion combines and organizes these earlier works in a single coherent framework, where the central role is played by the Bianchi identity (6.6) and the generalized Russian formula (6.10).

\section{Supersymmetric anomaly inflow from anomaly descent}

A direct connection between the supersymmetric anomaly inflow mechanism discussed in Sec. V and the generalized anomaly descent exists if and only if $X_{d+1}^{(1)}$ satisfies the normality condition (A8), i.e.,

$$
X_{d+1}^{(1)}=(\mathbf{s}+\mathbf{c}) Y_{d+1}^{(0)}+\mathrm{d} Z_{d}^{(1)},
$$

for some nontrivial $Y_{d+1}^{(0)} \neq W_{d+1}^{(0)}$. If $X_{d+1}^{(1)}$ can be expressed in this form, then the second descent equation in (A1) determines that

$$
(\mathbf{s}+\mathbf{c})\left(W_{d+1}^{(0)}-Y_{d+1}^{(0)}\right)=\mathrm{d}\left(Z_{d}^{(1)}-W_{d}^{(1)}\right),
$$

which allows us to identify $W_{d+1}^{(0)}-Y_{d+1}^{(0)}$ with a supersymmetric Chern-Simons form and $Z_{d}^{(1)}-W_{d}^{(1)}$ with the nontrivial solution of the WZ consistency conditions.

In order to verify that $Z_{d}^{(1)}-W_{d}^{(1)}$ coincides [up to $(\mathbf{s}+\mathbf{c})$-exact terms, i.e., local counterterms] with the solution of the WZ conditions obtained earlier in this section, we observe that the first descent equation in (A1) implies that

$$
\mathrm{d}\left((\mathbf{s}+\mathbf{c}) Z_{d}^{(1)}-X_{d}^{(2)}\right)=0,
$$

and hence,

$$
X_{d}^{(2)}=(\mathbf{s}+\mathbf{c}) Z_{d}^{(1)}+\mathrm{d} Z_{d-1}^{(2)} .
$$

We recognize this as the normality condition on $X_{d}^{(2)}$ we saw above. In particular, we identify

$$
Z_{d}^{(1)}=Y_{d}^{(1)}
$$

up to $(\mathbf{s}+\mathbf{c})$-exact terms. This confirms that the nontrivial solution of the WZ consistency conditions can be obtained from the Chern-Simons form $W_{d+1}^{(0)}-Y_{d+1}^{(0)}$ and takes the form

$$
\begin{aligned}
\text { anomaly }= & Z_{d}^{(1)}-W_{d}^{(1)}=Y_{d}^{(1)}-W_{d}^{(1)} \\
& +(\mathbf{s}+\mathbf{c}) \text {-exact terms. }
\end{aligned}
$$

To illustrate this connection between supersymmetric anomaly inflow and generalized descent, let us consider a simple example from Sec. V. From the supersymmetric anomaly inflow analysis in Sec. V we expect that $X_{d+1}^{(1)}$ can be expressed in normal form for all multiplets in $d$ dimensions that can be obtained by dimensional reduction from $d+1$ dimensions, without any further truncation. 
The 4D $\mathcal{N}=1$ flavor anomaly is therefore not a suitable example, since it must first be embedded in the $\mathcal{N}=2$ flavor multiplet in order to be obtainable from a $5 \mathrm{D}$ supersymmetric Chern-Simons action. The simplest example where $X_{d+1}^{(1)}$ can be expressed in normal form is the $\mathcal{N}=(1,1)$ flavor anomaly in $2 \mathrm{D}$ and the corresponding $\mathcal{N}=1$ supersymmetric Chern-Simons action in three dimensions.

The $\mathcal{N}=1$ vector multiplet in three dimensions can be obtained by consistent truncation of the $\mathcal{N}=3$ multiplet, as we discussed in Sec. V. Changing to anti-Hermitian generators, $t_{\mathrm{H} .}^{a} \rightarrow i t_{\mathrm{a} . \mathrm{H}}^{a}$, and scaling the gaugino and the supersymmetry parameter as

$$
\lambda^{a} \rightarrow i \lambda^{a}, \quad \epsilon \rightarrow \frac{i}{2} \epsilon,
$$

in order to match the conventions of the present section, the (canonically normalized) $\mathcal{N}=1$ supersymmetric ChernSimons form is given by

$$
\Omega_{\mathrm{CS}}=\operatorname{tr}\left(A \mathrm{~d} A+\frac{2}{3} A^{3}-\bar{\lambda} \lambda * \mathbb{1}\right)
$$

where we have fixed the choice of convention (5.13) by setting $\kappa=-1$.

The $\mathcal{N}=1$ supersymmetry transformations become

$$
\begin{aligned}
\delta_{Q}(\epsilon) A_{\mu} & =-\frac{1}{2} \bar{\epsilon} \gamma_{\mu} \lambda, \\
\delta_{Q}(\epsilon) \lambda_{\mu} & =\frac{1}{4}\left(\gamma^{\rho \sigma} \epsilon\right)_{\mu} F_{\rho \sigma},
\end{aligned}
$$

while the complete form of the corresponding BRST transformations is

$$
\begin{aligned}
\mathbf{s} A_{\mu} & =\mathcal{D}_{\mu} v, & \mathbf{c} A_{\mu} & =-\frac{1}{2} \bar{\alpha} \gamma_{\mu} \lambda^{a}+a^{\nu} \partial_{\nu} A_{\mu}, \\
\mathbf{s} \lambda & =\{\lambda, v\}, & \mathbf{c} \lambda & =\frac{1}{4} \gamma^{\mu \nu} F_{\mu \nu}^{a} \alpha+a^{\nu} \partial_{\nu} \lambda, \\
\mathbf{s} v & =-v^{2}, & \mathbf{c} v & =\frac{1}{4} \bar{\alpha} \gamma^{\mu} \alpha A_{\mu}+a^{\nu} \partial_{\nu} v, \\
\mathbf{s} \alpha & =0, & \mathbf{c} a^{\mu} & =-\frac{1}{4} \bar{\alpha} \gamma^{\mu} \alpha .
\end{aligned}
$$

From the definition of the generalized descent variables $X_{d+2-k}^{(k)}$ in (6.11) it follows that

$$
X_{d+1}^{(1)}=\frac{d+2}{2} P_{d+2}\left(\mathbf{c} A, F^{\frac{d}{2}}\right)
$$

In particular, for $d=2$ we have

$$
\begin{aligned}
X_{3}^{(1)} & =2 \operatorname{tr}(\mathbf{c} A F) \\
& =2 \operatorname{tr}\left(\left(-\frac{1}{2} \bar{\alpha} \gamma \lambda+\mathrm{d} i_{a} A+i_{a} \mathrm{~d} A\right) F\right) \\
& =2 \operatorname{tr}\left(-\frac{1}{2} \bar{\alpha} \gamma \lambda F+\mathrm{d}\left(i_{a} A\right) F+i_{a}\left(F-A^{2}\right) F\right) \\
& =\operatorname{tr}\left(-\bar{\alpha} \gamma \lambda F+2 \mathrm{~d}_{A}\left(i_{a} A\right) F+i_{a} F^{2}\right) \\
& =\operatorname{tr}(-\bar{\alpha} \gamma \lambda F)+\operatorname{dtr}\left(2 i_{a} A F\right),
\end{aligned}
$$

where in the last line we have used the fact that $F^{2}=0$ in three dimensions.

Moreover, using the BRST transformation of $\lambda$ we obtain

$$
\mathbf{c} \operatorname{tr}(\bar{\lambda} \lambda)=\frac{1}{2} \varepsilon^{\mu \nu \rho} \operatorname{tr}\left(\bar{\alpha} \gamma_{\rho} \lambda F_{\mu \nu}\right)+\partial_{\mu} \operatorname{tr}\left(a^{\mu} \bar{\lambda} \lambda\right),
$$

or in form notation

$$
\mathbf{c} \operatorname{tr}(\bar{\lambda} \lambda * \mathbb{1})=-\operatorname{tr}(\bar{\alpha} \gamma \lambda F)-\operatorname{dtr}(* a \bar{\lambda} \lambda) .
$$

We therefore conclude that $X_{3}^{(1)}$ can be expressed in normal form as

$$
X_{3}^{(1)}=(\mathbf{s}+\mathbf{c}) \operatorname{tr}(\bar{\lambda} \lambda * \mathbb{1})+\mathrm{d} \operatorname{tr}\left(2 i_{a} A F-* a \bar{\lambda} \lambda\right),
$$

from which we read off

$$
Y_{3}^{(0)}=\operatorname{tr}(\bar{\lambda} \lambda * \mathbb{1}), \quad Z_{2}^{(1)}=\operatorname{tr}\left(2 i_{a} A F-* a \bar{\lambda} \lambda\right) .
$$

Hence, the supersymmetric Chern-Simons form (6.58) is given by

$$
\Omega_{\mathrm{CS}}=W_{3}^{(0)}-Y_{3}^{(0)},
$$

in agreement with the general argument provided above.

\section{ACKNOWLEDGMENTS}

We thank H. Itoyama for communication on his pioneering work. We also acknowledge use of the symbolic computation package FieldsX [94] and I. P. would like to thank Markus Fröb for helpful correspondence and early access to an updated version of the package. The work of R. M. is supported in part by ERC Grants No. 772408Stringlandscape and No. 787320-QBH Structure. The work of I.P. and P. Y. is supported, respectively, by the KIAS Individual Grants No. PG064402 and No. PG005704 at the Korea Institute for Advanced Study.

\section{APPENDIX: ADDITIONAL NOTIONS}

In Sec. VI we have tried to simplify the discussion and avoid introducing new notions and definitions. However, as mentioned, our generalized descent procedure is inspired by earlier work on supersymmetric descent [14-18]. 
We collect here some definitions and ideas that have appeared in some form in the earlier literature and have been kept implicit in our presentation.

Without splitting the c part of the BRST operator $\mathrm{d}+\mathbf{s}+\mathbf{c}$ into parts and reserving $\mathbf{s}$ for the gauge transformations only, we can still recover the general pair of the descent towers for $X$ and $W$ outlined in Sec. VI,

$$
\begin{aligned}
& (\mathbf{s}+\mathbf{c}) X_{d+2-k}^{(k)}+\mathrm{d} X_{d+1-k}^{(k+1)}=0 \\
& X_{d+1-k}^{(k+1)}=(\mathbf{s}+\mathbf{c}) W_{d+1-k}^{(k)}+\mathrm{d} W_{d-k}^{(k+1)},
\end{aligned}
$$

which can be subject to further (nonuniversal) refinements.

\section{Filtration}

The descent relations we have discussed so far hold for any $\mathbf{c}$, as long as $(\mathrm{d}+\mathbf{s}+\mathbf{c})^{2}=0$. However, this structure is enriched when $\mathbf{c}$ possesses certain additional properties. One such case is when $\mathbf{s}+\mathbf{c}$ is itself nilpotent, i.e.,

$$
\mathbf{s}^{2}=0, \quad(\mathbf{s}+\mathbf{c})^{2}=0,
$$

where generically $\mathbf{c}^{2} \neq 0$, and both $\mathbf{s}$ and $\mathbf{c}$ anticommute with the exterior derivative d. This structure is formally known as a filtration of the BRST cohomology $[95,96]$ and it is practically very useful for computing the cohomology of $\mathbf{s}+\mathbf{c}$, given that of the simpler BRST operator $\mathbf{s}$. For example, as we discuss in Sec. III (see also [43] and references therein), the gravitational anomaly descent can be formulated in terms of the simpler descent for a $G L(d)$ gauge symmetry. More generally, the structure (A2) ensures that the cohomology of $\mathbf{s}+\mathbf{c}$ is a subspace of the cohomology of $\mathbf{s}$ (see Proposition 5.6 in [96]). The same holds for the cohomologies modulo d.

\section{Grading refinement}

It is often useful to further decompose the coefficients $X_{d+2-k}^{(k)}$ and $W_{d+1-k}^{(k)}$ in (A1), for example, according to different types of ghosts. In particular, we may distinguish between $\mathbf{s}$ and $\mathbf{c}$ ghosts by writing

$W_{d+1-k}^{(k)}=\sum_{l=0}^{k} W_{d+1-k}^{(l, k-l)}, \quad X_{d+2-k}^{(k)}=\sum_{l=0}^{k} X_{d+2-k}^{(l, k-l)}$,

where the first superscript corresponds to $\mathbf{s}$ ghosts and the second to $\mathbf{c}$ ghosts. However, such a decomposition is not always possible. This is the case, e.g., when $\mathbf{s}$ and $\mathbf{c}$ possess the nilpotency properties (A2), which imply that

$$
\mathbf{s c}+\mathbf{c s}+\mathbf{c}^{2}=0
$$

It follows that, in this case, unless the stronger conditions

$$
\mathbf{s c}+\mathbf{c s}=0, \quad \mathbf{c}^{2}=0,
$$

hold, the $\mathbf{s}$ and $\mathbf{c}$ ghost numbers are not separately conserved.

When a grading refinement of the form (A3) is possible, each descent relation in (A1) splits accordingly into a set of equations with definite $(p, q)$ ghost number. For example, for $k=1$ the first equation in (A1) splits into the three relations

$$
\begin{aligned}
\mathrm{d} X_{d}^{(2,0)}+\mathbf{s} X_{d+1}^{(1,0)} & =0, \quad \mathrm{~d} X_{d}^{(1,1)}+\mathbf{c} X_{d+1}^{(1,0)}=0, \\
\mathrm{~d} X_{d}^{(0,2)} & =0,
\end{aligned}
$$

since $X_{d+1}^{(0,1)}=0$. Similarly, the second equation in (A1) for $k=1$ splits into the relations

$$
\begin{aligned}
\mathbf{s} W_{d}^{(1,0)}+\mathrm{d} W_{d-1}^{(2,0)} & =0, \\
\mathbf{s} W_{d}^{(0,1)}+\mathbf{c} W_{d}^{(1,0)}+\mathrm{d} W_{d-1}^{(1,1)} & =-X_{d}^{(1,1)}, \\
\mathbf{c} W_{d}^{(0,1)}+\mathrm{d} W_{d-1}^{(0,2)} & =-X_{d}^{(0,2)} .
\end{aligned}
$$

As we discuss next, a grading refinement of the form (A3) is particularly useful when the coefficients $X_{d+2-k}^{(k)}$ satisfy two additional properties, namely "normality" and "orthogonality," which we now define.

\section{Normality}

Following [14], we say that $X_{d+2-k}^{(k)}$ is "normal" if it takes the form

$$
X_{d+2-k}^{(k)}=(\mathbf{s}+\mathbf{c}) Y_{d+2-k}^{(k-1)}+\mathrm{d} Z_{d+1-k}^{(k)},
$$

for some $Y_{d+2-k}^{(k-1)}$ and $Z_{d+1-k}^{(k)}$. Since the coefficients $X_{d+2-k}^{(k)}$ satisfy the descent relations (A1), this may seem like a tautology at first sight. However, the statement is not trivial in that one demands that $Y_{d+2-k}^{(k-1)}$ and $Z_{d+1-k}^{(k)}$ have different ghost content than respectively $W_{d+2-k}^{(k-1)}$ and $W_{d+1-k}^{(k)}$. This distinction becomes manifest when a grading refinement of the form (A3) is possible.

It is useful to notice that if the normality condition (A8) holds for $X_{d+2-k}^{(k)}$, then it also holds for all $X_{d+2-l}^{(l)}$ with $l>k$. This can be proven by induction, showing first that it holds for $l=k+1$. From (A1) follows that

$$
\begin{aligned}
0 & =\mathrm{d} X_{d+1-k}^{(k+1)}+(\mathbf{s}+\mathbf{c}) X_{d+2-k}^{(k)} \\
& =\mathrm{d}\left(X_{d+1-k}^{(k+1)}-(\mathbf{s}+\mathbf{c}) Z_{d+1-k}^{(k)}\right),
\end{aligned}
$$

and so

$$
X_{d+1-k}^{(k+1)}=(\mathbf{s}+\mathbf{c}) Y_{d+1-k}^{(k)}+\mathrm{d} Z_{d-k}^{(k+1)},
$$

with $Y_{d+1-k}^{(k)}=Z_{d+1-k}^{(k)}$. Hence, $X_{d+1-k}^{(k+1)}$ is also normal. This completes the proof. 


\section{Orthogonality}

We have seen that the Russian formula (2.15) ensures that the coefficient $X_{d+1}^{(1)}$ contains only ghosts associated with the operator c. If this property extends to all higher order coefficients, we say that $X_{d+1-k}^{(k+1)}$ are "orthogonal." Clearly, a prerequisite for this property is that a grading refinement of the form (A3) exists. The orthogonality condition can be stated as

$$
X_{d+2-k}^{(l, k-l)}=0, \quad \forall l \neq 0,
$$

or equivalently

$$
X_{d+2-k}^{(k)}=X_{d+2-k}^{(0, k)} .
$$

From the definition (6.11) of the coefficients $X_{d+2-k}^{(k)}$ follows that this property is guaranteed provided the difference $\hat{\mathcal{G}}-\mathcal{F}$ between the field strengths does not depend on the gauge ghost $v$. This condition can be used as a possible criterion for the choice of the shift $u$ in the generalized field strength (6.5). Notice that if the coefficients $X_{d+2-k}^{(k)}$ are also normal, then the definition (A8) requires that

$$
Y_{d+2-k}^{(k-1)}=Y_{d+2-k}^{(0, k-1)}, \quad \mathbf{s} Y_{d+2-k}^{(k-1)}=0, \quad Z_{d+1-k}^{(k)}=Z_{d+1-k}^{(0, k)}
$$

In the main part of the paper we have opted for the choice $u=0$ in order to ensure the uniform description of diffeomorphisms and supersymmetry.
[1] S. L. Adler, Axial vector vertex in spinor electrodynamics, Phys. Rev. 177, 2426 (1969).

[2] W. A. Bardeen, Anomalous Ward identities in spinor field theories, Phys. Rev. 184, 1848 (1969).

[3] J. S. Bell and R. Jackiw, A PCAC puzzle: $\pi^{0} \rightarrow \gamma \gamma$ in the $\sigma$ model, Nuovo Cimento A 60, 47 (1969).

[4] K. Fujikawa, Path Integral Measure for Gauge Invariant Fermion Theories, Phys. Rev. Lett. 42, 1195 (1979).

[5] L. Alvarez-Gaume and E. Witten, Gravitational anomalies, Nucl. Phys. B234, 269 (1984).

[6] W. A. Bardeen and B. Zumino, Consistent and covariant anomalies in gauge and gravitational theories, Nucl. Phys. B244, 421 (1984).

[7] O. Piguet, K. Sibold, and M. Schweda, General solution of the supersymmetry consistency conditions, Nucl. Phys. B174, 183 (1980).

[8] H. Itoyama, V. P. Nair, and H.-c. Ren, Supersymmetry anomalies and some aspects of renormalization, Nucl. Phys. B262, 317 (1985).

[9] E. Guadagnini and M. Mintchev, Chiral anomalies and supersymmetry, Nucl. Phys. B269, 543 (1986).

[10] H. Itoyama, V. P. Nair, and H.-c. Ren, Supersymmetry anomalies: Further results, Phys. Lett. 168B, 78 (1986).

[11] G. Girardi, R. Grimm, and R. Stora, Chiral anomalies in $N=1$ supersymmetric Yang-Mills theories, Phys. Lett. 156B, 203 (1985).

[12] D. S. Hwang, Supersymmetry anomaly in two-dimensions, Nucl. Phys. B267, 349 (1986).

[13] P. Altevogt and B. Rieger, Chiral anomalies in $N=1$ supersymmetric Yang-Mills theories in two-dimensions, Nuovo Cimento A 97, 451 (1987).

[14] R. Kaiser, Supersymmetric gauge anomalies in twodimensions and four-dimensions from generalized descent equations, Z. Phys. C 39, 585 (1988).

[15] P. Altevogt and R. Kaiser, Supersymmetric gauge anomalies in two-dimensions, four-dimensions, six-dimensions and ten-dimensions, Z. Phys. C 43, 455 (1989).
[16] P. Altevogt and R. Kaiser, The ten-dimensional supersymmetric gauge anomaly, Phys. Lett. B 219, 464 (1989).

[17] L. Baulieu, G. Bossard, and S. P. Sorella, Shadow fields and local supersymmetric gauges, Nucl. Phys. B753, 273 (2006).

[18] L. Baulieu and A. Martin, Supersymmetric Adler-Bardeen anomaly in $N=1$ super-Yang-Mills theories, Phys. Lett. B 670, 165 (2008).

[19] A. Bzowski, G. Festuccia, and V. Procházka, Consistency of supersymmetric 't Hooft anomalies, J. High Energy Phys. 02 (2021) 225.

[20] Y. Tanii, Local supersymmetry anomaly in two-dimensions, Nucl. Phys. B259, 677 (1985).

[21] P. S. Howe and P.C. West, Gravitational anomalies in supersymmetric theories, Phys. Lett. 156B, 335 (1985).

[22] S. J. Gates, Jr., M. T. Grisaru, and W. Siegel, Auxiliary field anomalies, Nucl. Phys. B203, 189 (1982).

[23] B. de Wit and M. T. Grisaru, Compensating fields and anomalies, in Quantum Field Theory and Quantum Statistics, edited by I. A. Batalin et al. (Adam Hilger, Bristol, UK, 1987), Vol. 2, pp. 411-432.

[24] I. Papadimitriou, Supercurrent anomalies in 4d SCFTs, J. High Energy Phys. 07 (2017) 038.

[25] O. S. An, Anomaly-corrected supersymmetry algebra and supersymmetric holographic renormalization, J. High Energy Phys. 12 (2017) 107.

[26] I. Papadimitriou, Supersymmetry anomalies in $\mathcal{N}=1$ conformal supergravity, J. High Energy Phys. 04 (2019) 040.

[27] I. Papadimitriou, Supersymmetry anomalies in new minimal supergravity, J. High Energy Phys. 09 (2019) 039.

[28] O. S. An, J. U. Kang, J. C. Kim, and Y. H. Ko, Quantum consistency in supersymmetric theories with $R$-symmetry in curved space, J. High Energy Phys. 05 (2019) 146.

[29] S. M. Kuzenko, A. Schwimmer, and S. Theisen, Comments on anomalies in supersymmetric theories, J. Phys. A 53, 064003 (2020). 
[30] G. Katsianis, I. Papadimitriou, K. Skenderis, and M. Taylor, Anomalous Supersymmetry, Phys. Rev. Lett. 122, 231602 (2019).

[31] G. Katsianis, I. Papadimitriou, K. Skenderis, and M. Taylor, Supersymmetry anomaly in the superconformal Wess-Zumino model, J. High Energy Phys. 02 (2021) 209.

[32] K. Nakagawa and Y. Nakayama, $C P$-violating supersymmetry anomaly, Phys. Rev. D 104, 025018 (2021).

[33] L. Bonora, P. Pasti, and M. Tonin, The chiral anomaly in supersymmetric gauge theories coupled to supergravity, Phys. Lett. 167B, 191 (1986).

[34] F. Brandt, Anomaly candidates and invariants of $D=4$, $N=1$ supergravity theories, Classical Quantum Gravity 11, 849 (1994).

[35] F. Brandt, Local BRST cohomology in minimal $D=4$, $N=1$ supergravity, Ann. Phys. (N.Y.) 259, 357 (1997).

[36] C. Closset, L. Di Pietro, and H. Kim, 't Hooft anomalies and the holomorphy of supersymmetric partition functions, J. High Energy Phys. 08 (2019) 035.

[37] M. F. Atiyah and I. M. Singer, Dirac operators coupled to vector potentials, Proc. Natl. Acad. Sci. U.S.A. 81, 2597 (1984).

[38] L. D. Faddeev and S. L. Shatashvili, Algebraic and hamiltonian methods in the theory of non-Abelian anomalies, Teor. Mat. Fiz. 60, 206 (1984).

[39] J. Manes, R. Stora, and B. Zumino, Algebraic study of chiral anomalies, Commun. Math. Phys. 102, 157 (1985).

[40] C. G. Callan, Jr. and J. A. Harvey, Anomalies and fermion zero modes on strings and domain walls, Nucl. Phys. B250, 427 (1985).

[41] C. Becchi, A. Rouet, and R. Stora, Renormalization of gauge theories, Ann. Phys. (N.Y.) 98, 287 (1976).

[42] Y. Kosman, Dérivées de Lie des spineurs, Ann. Mat. 91, 317 (1971).

[43] R. Bertlmann, Anomalies in Quantum Field Theory (Oxford University Press, New York, 1996).

[44] K. Jensen, R. Loganayagam, and A. Yarom, Thermodynamics, gravitational anomalies and cones, J. High Energy Phys. 02 (2013) 088.

[45] M. J. Duff, J. T. Liu, and R. Minasian, Eleven-dimensional origin of string-string duality: A one loop test, Nucl. Phys. B452, 261 (1995).

[46] E. Witten, Five-branes and M theory on an orbifold, Nucl. Phys. B463, 383 (1996).

[47] M. B. Green, J. A. Harvey, and G. W. Moore, I-brane inflow and anomalous couplings on d-branes, Classical Quantum Gravity 14, 47 (1997).

[48] Y.-K. E. Cheung and Z. Yin, Anomalies, branes, and currents, Nucl. Phys. B517, 69 (1998).

[49] R. Minasian and G. W. Moore, K theory and RamondRamond charge, J. High Energy Phys. 11 (1997) 002.

[50] D. Freed, J. A. Harvey, R. Minasian, and G. W. Moore, Gravitational anomaly cancellation for $\mathrm{M}$ theory fivebranes, Adv. Theor. Math. Phys. 2, 601 (1998).

[51] H. Kim and P. Yi, D-brane anomaly inflow revisited, J. High Energy Phys. 02 (2012) 012.

[52] H.-C. Kao and K.-M. Lee, Selfdual Chern-Simons systems with an $N=3$ extended supersymmetry, Phys. Rev. D 46, 4691 (1992).
[53] H.-C. Kao, K.-M. Lee, and T. Lee, The Chern-Simons coefficient in supersymmetric Yang-Mills Chern-Simons theories, Phys. Lett. B 373, 94 (1996).

[54] D. Z. Freedman and A. Van Proeyen, Supergravity (Cambridge University Press, Cambridge, England, 2012).

[55] K. Shima and M. Tsuda, Linearizing $N=3$ nonlinear supersymmetry in two dimensions, Phys. Lett. B 641, 101 (2006).

[56] T. Kugo and K. Ohashi, Off-shell $D=5$ supergravity coupled to matter Yang-Mills system, Prog. Theor. Phys. 105, 323 (2001).

[57] V. Cortes, C. Mayer, T. Mohaupt, and F. Saueressig, Special geometry of Euclidean supersymmetry. 1. Vector multiplets, J. High Energy Phys. 03 (2004) 028.

[58] S. M. Kuzenko and J. Novak, On supersymmetric ChernSimons-type theories in five dimensions, J. High Energy Phys. 02 (2014) 096.

[59] L. Gall and T. Mohaupt, Five-dimensional vector multiplets in arbitrary signature, J. High Energy Phys. 09 (2018) 053.

[60] S. Ferrara and P. van Nieuwenhuizen, The auxiliary fields of supergravity, Phys. Lett. 74B, 333 (1978).

[61] K. Stelle and P.C. West, Minimal auxiliary fields for supergravity, Phys. Lett. 74B, 330 (1978).

[62] E. Fradkin and M. A. Vasiliev, S matrix for theories that admit closure of the algebra with the aid of auxiliary fields: The auxiliary fields in supergravity, Lett. Nuovo Cimento 22, 651 (1978).

[63] V. P. Akulov, D. V. Volkov, and V. A. Soroka, On the general covariant theory of calibrating poles in superspace, Theor. Math. Phys. 31, 285 (1977).

[64] M. F. Sohnius and P. C. West, An alternative minimal offshell version of $N=1$ supergravity, Phys. Lett. 105B, 353 (1981).

[65] M. Sohnius and P. C. West, The tensor calculus and matter coupling of the alternative minimal auxiliary field formulation of $N=1$ supergravity, Nucl. Phys. B198, 493 (1982).

[66] S. Ferrara and S. Sabharwal, Structure of new minimal supergravity, Ann. Phys. (N.Y.) 189, 318 (1989).

[67] G. Girardi, R. Grimm, M. Muller, and J. Wess, Antisymmetric tensor gauge potential in curved superspace and a $(16+16)$ supergravity multiplet, Phys. Lett. 147B, 81 (1984).

[68] W. Lang, J. Louis, and B. A. Ovrut, $(16+16)$ supergravity coupled to matter: The low-energy limit of the superstring, Phys. Lett. 158B, 40 (1985).

[69] W. Siegel, 16/16 Supergravity, Classical Quantum Gravity 3, L47 (1986).

[70] M. Kaku, P. K. Townsend, and P. van Nieuwenhuizen, Gauge theory of the conformal and superconformal group, Phys. Lett. 69B, 304 (1977).

[71] M. Kaku, P. K. Townsend, and P. van Nieuwenhuizen, Superconformal Unified Field Theory, Phys. Rev. Lett. 39, 1109 (1977).

[72] M. Kaku, P. K. Townsend, and P. van Nieuwenhuizen, Properties of conformal supergravity, Phys. Rev. D 17, 3179 (1978).

[73] P. K. Townsend and P. van Nieuwenhuizen, Simplifications of conformal supergravity, Phys. Rev. D 19, 3166 (1979). 
[74] Z. Komargodski and N. Seiberg, Comments on supercurrent multiplets, supersymmetric field theories and supergravity, J. High Energy Phys. 07 (2010) 017.

[75] P. van Nieuwenhuizen, $D=3$ conformal supergravity and Chern-Simons terms, Phys. Rev. D 32, 872 (1985).

[76] M. Rocek and P. van Nieuwenhuizen, $\mathcal{N} \geq 2$ supersymmetric Chern-Simons terms as $d=3$ extended conformal supergravity, Classical Quantum Gravity 3, 43 (1986).

[77] M. Cederwall, U. Gran, and B. E. W. Nilsson, $D=3, N=8$ conformal supergravity and the dragon window, J. High Energy Phys. 09 (2011) 101.

[78] M. Nishimura and Y. Tanii, Coupling of the BLG theory to a conformal supergravity background, J. High Energy Phys. 01 (2013) 120.

[79] S. M. Kuzenko and G. Tartaglino-Mazzucchelli, Conformal supergravities as Chern-Simons theories revisited, J. High Energy Phys. 03 (2013) 113.

[80] D. Butter, S. M. Kuzenko, J. Novak, and G. TartaglinoMazzucchelli, Conformal supergravity in three dimensions: New off-shell formulation, J. High Energy Phys. 09 (2013) 072.

[81] D. Butter, S. M. Kuzenko, J. Novak, and G. TartaglinoMazzucchelli, Conformal supergravity in three dimensions: Off-shell actions, J. High Energy Phys. 10 (2013) 073.

[82] M. Nishimura and Y. Tanii, $N=6$ conformal supergravity in three dimensions, J. High Energy Phys. 10 (2013) 123.

[83] S. M. Kuzenko, J. Novak, and G. Tartaglino-Mazzucchelli, $N=6$ superconformal gravity in three dimensions from superspace, J. High Energy Phys. 01 (2014) 121.

[84] A. Achucarro and P. K. Townsend, A Chern-Simons action for three-dimensional anti-De Sitter supergravity theories, Phys. Lett. B 180, 89 (1986).
[85] A. Achucarro and P. K. Townsend, Extended supergravities in $d=2+1$ as Chern-Simons theories, Phys. Lett. B 229, 383 (1989).

[86] M. Nishimura and Y. Tanii, Super Weyl anomalies in the AdS/CFT correspondence, Int. J. Mod. Phys. A 14, 3731 (1999).

[87] M. Banados, A. Schwimmer, and S. Theisen, Chern-Simons gravity and holographic anomalies, J. High Energy Phys. 05 (2004) 039.

[88] S. N. Solodukhin, Holographic description of gravitational anomalies, J. High Energy Phys. 07 (2006) 003.

[89] M. Henningson and K. Skenderis, The holographic Weyl anomaly, J. High Energy Phys. 07 (1998) 023.

[90] S. J. Gates, Jr., Y. Hassoun, and P. van Nieuwenhuizen, Auxiliary fields for $d=2, N=4$ supergravity, Nucl. Phys. B317, 302 (1989).

[91] A. H. Chamseddine and J. Frohlich, Two-dimensional Lorentz-Weyl anomaly and gravitational Chern-Simons theory, Commun. Math. Phys. 147, 549 (1992).

[92] C. Imbimbo and D. Rosa, The topological structure of supergravity: An application to supersymmetric localization, J. High Energy Phys. 05 (2018) 112.

[93] M. B. Fröb, C. Imbimbo, and N. Risso, Deformations of supergravity and supersymmetry anomalies, J. High Energy Phys. 12 (2021) 009.

[94] M. B. Fröb, FieldsX-An extension package for the xAct tensor computer algebra suite to include fermions, gauge fields and BRST cohomology, arXiv:2008.12422.

[95] J. A. Dixon, Calculation of BRS cohomology with spectral sequences, Commun. Math. Phys. 139, 495 (1991).

[96] O. Piguet and S. Sorella, Algebraic renormalization: Perturbative renormalization, symmetries and anomalies, Lect. Notes Phys., M: Monogr. 28, 1 (1995). 